\title{
THE CLASSIFICATION OF GESTURE INTERACTIONS AND THE STUDY OF THEIR ERGONOMIC EFFECT ON HANDS
}

by

\author{
Yu Wai Chau \\ Bachelor of Technology, \\ RCC Institute of Technology 2013
}

\author{
A thesis presented to \\ Ryerson University \\ in partial fulfillment of the \\ requirements for the degree of \\ Master of Applied Science \\ in the Program of \\ Electrical and Computer Engineering
}

Toronto, Ontario, Canada, 2017

(C) Yu Wai Chau 2017 
I hereby declare that I am the sole author of this thesis.

I authorize Ryerson University to lend this thesis to other institutions or individuals for the purpose of scholarly research.

I further authorize Ryerson University to reproduce this thesis by photocopying or by other means, in total or in part, at the request of other institutions or individuals for the purpose of scholarly research.

I understand that my thesis may be made electronically available to the public. 
The Classification of Gesture Interactions and the Study of their Ergonomic Effects on Hands

\author{
Master of Applied Science 2017 \\ Yu Wai Chau \\ Electrical and Computer Engineering \\ Ryerson University
}

\begin{abstract}
In order to investigate gestural behavior during human-computer interactions, an investigation into the designs of current interaction methods is conducted. This information is then compared to current emerging databases to observe if the gesture designs follow guidelines discovered in the above investigation. The comparison will also observe common trends in the currently developed gesture databases such as similar gesture for specific commands. In order to investigate gestural behavior during interactions with computer interfaces, an experiment has been devised to observe and record gestures in use for gesture databases through the use of a hardware sensor device. It was discovered that factors such as opposing adjacent fingers and gestures that simulated object manipulation are factors in user comfort. The results of this study will create guidelines for creating new gestures for hand gesture interfaces.
\end{abstract}




\section{Acknowledgments}

I would like to give thanks to my supervisor Dr. Kristina Mai for supporting and guiding me during my studies in the Master of Applied Sciences in Electrical and Computer Engineering program, who provided me with valuable ideas and resources during the creation of this thesis.

I would like to give my loving thanks to my mother and sister, without whom this work would not be possible, for their support, care, and love during my studies. Their belief in me was inspirational. 


\section{Table of Contents}

Declaration $\quad$ ii

Abstract iii

Acknowledgements

List of Tables $\quad$ vii

List of Figures viii

List of Appendices $\quad$ ix

1 Introduction 1

$1.1 \quad$ Background 1

1.2 Purpose and Scope 2

1.3 Contributions 3

1.4 Overview of Thesis 4

2 Gesture Based Computer Interfaces $\quad 5$

2.1 Gesture Recognition Methods 5

2.1.1 Image Recognition Techniques 6

2.1.2 Data Management 8

2.1.3 Specialty Hardware 9

2.2 Gesture Classification 11

$2.3 \quad$ Gesture and Language 13

2.4 Designs of Gestures 15

$\begin{array}{lll}2.5 & \text { Summary } & 18\end{array}$

$3 \quad$ Gesture Design Investigative Methodology 19

3.1 Gesture Types $\quad 21$

3.1.1 Static Gestures 23

3.1.2 Dynamic Gestures 24

3.2 Gesture Designs 25

3.2.1 Exertion 25

$\begin{array}{lll}3.2 .2 & \text { Position } & 30\end{array}$ 
$4 \quad$ Experiments and Results $\quad 35$

4.1 Gesture Categorization 36

4.2 Gesture Exertion on User 48

4.2.1 High Exertion $\quad 51$

4.2.2 Low Exertion 53

$\begin{array}{lll}4.3 & \text { Summary } & 56\end{array}$

$5 \quad$ Discussions and Conclusions $\quad 58$

5.1 Design Guidelines $\quad 58$

$\begin{array}{lll}5.2 & \text { Possible Future Work } & 60\end{array}$

$\begin{array}{lll}5.3 \text { Conclusion } & 61\end{array}$

References 


\section{List of Tables}

Table 3.1: Rest and Exertion point reference table (Activation Bits) 29

Table 3.2: Rest and Exertion point reference table (Normalized) 29

Table 3.3: Rest, flex and clenched reference

Table 3.4: Range assigned to each value by degrees $\left(\mathrm{X}_{0}\right)$

Table 4.1: Categorization of compiled database gestures 47

Table 4.2: High Exertion Gestures (Activity Levels) $\quad 51$

Table 4.3: High Exertion Gestures Movement Range 52

Table 4.4: Low Exertion Gestures (Activity Levels) 53

Table 4.5: Low Exertion Gesture Movement Range 55

Table 4.1: Compiled Database Gestures $\quad 72$

Table 4.2: American Sign Language A-Z

Table 3.1: Rest and Exertion Points Reference Table $\quad 79$

Table 4.1: Myo Armband sensor Readings Static (Activity Levels) 80

Table 4.2: Static Gesture Myo Armband Readings Normalized $\quad 81$

Table 4.3: Myo Armband sensor Readings Dynamic 82

Table 4.4: Dynamic Gesture Myo Armband Readings Normalized 83

Table 4.5: Myo Armband EMG Plots 94

Table 3.2: Rest, flex and clenched reference table 95

Table 4.6: Movement Range Table Static 96

Table 4.7: Movement Range Table Dynamic 97 


\section{List of Figures}

Figure 3.1: Example of iconic gestures, American Sign Language letters ASL 21

Figure 3.2: Example of Metaphoric gesture, the spreading of the hand 22

Figure 3.3: Example of deictic gesture, the pointing gesture 22

Figure 3.4: An example of a static gesture 23

Figure 3.5: Example of dynamic gesture, wave out 24

Figure 3.6: Myo Armband pod numbering 26

Figure 3.7: Myo EMG reading of a closed fist 28

Figure 3.8: Sensor Data Rectifying, Rounding, Averaging Algorithm 30

Figure 3.9: Relaxed hand used in experiments (Reference 0, 90 $) 31$

Figure 3.10: Closed fist used in experiments (Reference -10,180 ) 31

Figure 3.11: Fully extended open hand used in experiments (Reference 10, 00) 32

Figure 3.12: Position Measuring Block Diagram; (A) Static, (B) Dynamic 33

Figure 4.1: Myo Armband EMG reading for full exertion $\quad 48$

Figure 4.2: Myo Armband EMG readings for: ASL B and ASL C 49

Figure 4.3: Myo Armband EMG readings for: Tilt, Fork Tilt, Palm and Moving Point 50

Figure 4.4: Finger Difference vs Exertion (Dynamic) 56 


\section{List of Appendices}

Compiled Gesture Database

Myo Sensor Data

Gesture Movement Rating 


\section{Chapter 1}

\section{Introduction}

\section{1 Background}

With the advancements of new image processing algorithms and powerful processors, the ability to recognize shapes and interpret them in real time is a possibility. This has given rise to the growing field of gesture recognition for human-computer interactions. Due to the complexity of human-human communications, computers must rely on a rigid standard for communication that is both simple and robust. However this effectively limits the amount of information that can transfer to the computer as humans can naturally output more information through speech, body language and hand gestures. With the addition of gesturing in human-computer interaction, more information can be transferred and due to the spatial nature of gestures 3D dimensional concepts can be more intuitively conveyed by the user. However one obstacle in implementation of these systems is that they are still in their infancy with new methods being developed to eliminate or minimize current weaknesses. As the technology is still in its infancy, its rarity has given rise mostly to novelty in its applications. As a rare element that is being developed, little studies have been performed into how the interaction should be developed as the main focus of the technology currently is in the computer systems. 
The use of gesture-recognition in human-computer interactions offers distinct and decisive advantages to current methods. The main advantage offered is a physical quantity can be more quickly and effectively conveyed by quantities such as shape, movement, and physical behaviors. Studies in human cognitive functions have shown that speech can be supplemented with gestures to convey a clearer meaning to the recipient which would in turn help in speech recognition in machines and computing systems.

Gesture Recognition enjoys two recognition methods that are currently in development; those of hardware sensor devices and image processing techniques. Image processing techniques make use of simple color cameras that are widely distributed while hardware sensors make use of a suite of sensory devices to obtain gesture information. An example of such a device is the Myo Armband which makes use of Electromyography (EMG) sensors, accelerometers, and gyroscopes to get position and hand shape to facilitate in its gesture recognition. However while hardware sensors offer an advantage in accuracy, the widespread distribution of color cameras make image processing have a distinct advantage over hardware sensors.

Currently the technology is developed per application as each application of gesturerecognition is developed to communicate a specific purpose. Much like text recognition and speech recognition, a fixed set of text or words is used to communicate in clear rigid understanding of the intents and directed actions of the user. In most cases gesture-recognition is typically designed for gaming application with some parties interested in design applications. This would limit the growth and potential of this technology. As one of the primary means that humans use to communicate with each other, limiting this technology to such specific applications will also limit our ability to communicate with computers more effectively. Some focus should be shifted into the development of how to clearly communicate with computers intuitively.

\subsection{Objective and Scope}

The goal of this paper is to observe the current development of gestures used when creating gesture databases for recognition and their interpretations and to provide a framework for future endeavors to develop proper general purpose recognition databases. The study will include 
current public sources and their study samples. This paper will categorize gestures based on similarities in shape and intents to create general cases for specific and basic commands that a computer should recognize to have meaningful communication. Additionally, the exertion on the user will be investigated to provide a framework for ergonomic designs and considerations. Though this investigation, the goal of this thesis is to improve the design process of gesture recognition systems as well as help achieve better public support through better design goals and improved alternative computing system inputs.

The intentions are not to simply replace current existing methods, but to enhance, substitute and supplement current communication methods. The long-term goal of this paper is to provide a compare and contrast the current interaction methods to provide a base to which new design considerations can be drawn from. To accomplish this, this thesis will focus on gesture categorization in an effort to break down and separate gestures to facilitate better understanding of them individually and to help organize results from other work this thesis will conduct. Gestures will then be investigated though their ergonomic effects of exertion and positions. Through this study traits and features of gestures can be recognized as harmful or injuring of gesture practitioners of the emerging technology. By devising a method to view exertion by the user on gesture making, it is possible to demonstrate the effect of gestures on a user relative to their abilities and physical characteristics.

\subsection{Contributions}

This work contributes in the following:

Through investigation into gesture category with respect to usage, design, meaning, and interpretations, a better understanding of gestures and the expectations of the designs can be observed and used to match with user expectations. This allows studies to be conducted to find if a target gesture, either static or dynamic is suitable for the command or if an alternative is available.

In the study of exertion with respect to finger position and movement ranges a system to observe and quantize exertion on users was created; through this system studies can observe and 
discover characteristics for both harmful gestures and sustainable gestures to facilitate better guidelines with designing gesture databases.

Using the system above and a compilation of various in research databases, several characteristics and guidelines were produce to demonstrate the success of the research. The research was able to identify key physical characteristics of gesture making for both exertive and restive gestures and produce guidelines to minimize exertion experienced by users. More extensive work and variety in this study could produce a better understanding and design rules when creating a system that utilizes human gesture inputs.

\subsection{Overview of thesis}

In Chapter 2 of this paper, current developments in the field will be examined; recognition methods, gesture types, gesture categories, and gesture ergonomics will be studied. Chapter 3 will outline the method of analysis for static and dynamic gestures as well as the exertion with respect to the degree of flex the user experiences. Chapter 4 will discuss the results and examine the characteristics and discoveries found using the method listed above. Chapter 5 will summarize the analysis and propose future work and methods that can be undertaken.

This work will look at recognition methods primarily taken from single camera based systems that implement color and pattern recognition. This is done because the hardware for this application has already seen widespread distribution and thus has the largest potential to reach the highest number of users. Perhaps the most investigated method of recognition is shape and contour classification, given that this method ignores skin tones, background texture, and color contrast in the image. It has also been shown to have the highest success rate given the diversity of users in size, skin tone, and environment and variety applications.

This work will study and observe current gesture databases in order to investigate the nature of the gestures as well as observe its effects on the user. The primary focus of this work is to observe the function of the gesture, the type and its ergonomic impact on the user. This will set a guideline on how to approach gesture design. To accomplish this, a Myo Armband will be employed to obtain EMG readings. 


\section{Chapter 2}

\section{Gesture Based Computer Interfaces}

As interactions with technology reaches beyond the bounds of Graphical User Interfaces, the need for interaction between Human and Computer to become more natural is growing [12]. While physical sensor on the user will produce accurate measurements and eliminate errors in reading hand positions, vision based system provide a closer approximation to human to human interaction [9]. In order to ensure computer systems have the resources to ensure fast or real time processing speeds, a general framework is common among most recognition software. The main components of which are segmentation, feature extraction and then recognition [41]. Some of the design considerations when creating a recognition system are the specific use; this may involve different peripherals such as infrared sensors, lighting fixtures, and Z-axis considerations [55].

\subsection{Gesture Recognition Methods}

Typical vision recognition systems use variety of techniques to recognize gestures. The following are state of the art techniques employed by current research. The most basic these techniques employs the use of RBG color cameras using only color and light spectrum to process the shapes and hand gestures. However, some techniques proposed to employ the use of specialty hardware in conjunction with the RBG color camera to enhance the image processing. 
The Microsoft Kinect is often cited as a prime example of improved recognition techniques at a reasonable cost [44]. Below are recognition algorithms and systems organized by traits; in section 2.1.1 recognition methods that use image characteristics are examined, in 2.1.2, data comparison and retrieval will be examined and in 2.1.3 specialty hardware proposed for improved performance will be investigated.

\subsubsection{Image Recognition Techniques}

Discriminating Features: The method in which the information will be extracted is as follows: First the RGB image and depth will be extracted, and then the histogram of oriented gradients and local binary patterns features are extracted. To effectively extract information, only dynamic regions will be examined [9]. Some algorithms classify and look for details such as finger tips and joints to better recognize gestures. However, this method is extremely difficult as the features may experience collisions and/occlusions. Other features that algorithms may track are 3D features which track a 3D model of the hand relative to specific key features the algorithm looks for [29] [39] [41].

Urdu Alphabets using Geometrical Classification: This system looks to extract features from the hand by color coordination, by coloring different features of the hand in color; the algorithm can track and recognize features more effectively. As demonstrated in the study, the algorithm is able to distinguish the orientation of the fingers as the fronts of the fingers are colored blue while the back is colored red [6].

Shape Parameters: In this method, the followings steps are taken when processing the gesture images, Image Segmentation, Orientation Detection, Feature Extraction, Feature Classification, Bits Generation and finally Gesture-Interpretation. The main feature in this method is the shape the hand is making towards the camera; as such features such as thumb and fingers are more thoroughly examined for interpretation [5]. This method is also called low-level feature as the algorithms observe the contours, shape, or image and compares it to a sample in the database. Though it offers robust and noise resistance though its major drawback is the restriction of backgrounds the system can be used in [29]. Another use of the shape parameter is to supplement the use of dynamic gesture recognition as they can help the system recognize the 
start and the end point of the gesture [53].

Sub Gesture Modeling: In this method the main feature is the flow of the movements, that is, that gestures, much like sentences, are comprised of smaller sub-gestures. As a measure to ensure high recognition chances, the method discusses "start" and "end" phases to gestures [4]. Another description of this type of recognition is a temporally ordered set of states representing spatial information at the scene [44].

Dynamic Time Warping: This technique shares similarities with speech recognition in that the process is a similarity measure of the input signal to that of a sample in the database [7].

Local Motion Signatures of histogram of oriented gradients descriptors: The basic frame work for the system is as follows: the first step is to compute the features in the scene, then for each feature, associate a 2D descriptor which is then tracked over time to create a local motion signature. The signatures are then clustered into local motion patterns, the system then extracts the local motion signatures and then voting on what is the most likely gesture from the patterns [11].

Contour Classification: This approach identifies each static gesture as a unique set of contours that can be represented by a series of equations. By filtering each image until only the basic shape of the gesture is obtained and then finding all the contours of the image will create a robust and simple approach to recognition. Furthermore, the use of this technique allows for a system that uses minimal processing to find the related gesture within the database [3]. Another method of using Contour classification is the use of finger counting algorithms based on the shape of the hand. Due to the simple nature of this system, classification and retrieval of the gesture is relatively quick and robust. However, in its current form, the system is only able to recognize a small sample with large distinctions between each gesture [30].

Depth Camera Recognition: Using a metric called the Finger-Earth Mover's Distance to measure hand shape dissimilarities, the accuracy of the recognition system was vastly improved. It has been shown that the metric allows for better shape recognition in terms of speed and accuracy. This allows the system to have better performance in real time [29]. 


\subsubsection{Data Management}

Gesture Vocabulary: One such method of recognition is to use the vocabulary of the system's gesture language design as a method for recognition and data retrieval. The use of logical gesture sequencing can reduce error and computing power. These are characteristics noted in Gesture Vocabulary studies for both robotic and human vocabularies.

1. Human: Due to the nature of this type of gesture being innate to human nature, there are no predefined limits on designs of this type of gesture [54].

2. Robot: Participants of the study found that gestures most associated with a specific task is generally the best gesture for that response, however, the study notes that cultural differences may differ the meaning of the gesture [54].

Database Query: As gestures are a series of body silhouettes one proposed method of gesture recognition is to extract global feature from a continuous input from the user then adopting a set of descriptors for matching [28].

Neural network: In order to make gesture recognition more intuitive and ease of use, a proposed neural network has been proposed to enable the system to learn and adjust to new gestures, changes, and minor variations. The problem this approach brings forth is the issue of weights, in the network, current methods generate local minimums and new inputs learned causes the system to forget older ones. The experiments in the article sought to eliminate and minimize these issues [59].

One application of gesture recognition is for security purposes. In the study performed in [34], the use of gesture recognition for electronic examination is considered. To improve security and uphold academic integrity, the study demonstrates that each person has a unique habit during examination. By using gesture recognition to identify the user in question and to monitor action taken by the user, the system is able to identify if the current user is the initial user. The study also proposes that the system, if developed further, can be used to ensure the user does not cheat but looking through a book or browsing through online sources [34]. Another approach to 
use gesture recognition as security is its use in place of pass codes. A study was conducted using different user bio-metrics to identify a user. By having the user perform a specific gesture, the system can observe and identify similarities to the sample taken previously. Such a measure can

be used to ensure better security as another user will have to obtain similar bio-metrics to another user in the database [36].

\subsubsection{Specialty Hardware}

Gaze and Touch Gestures, are among some of the new methods of improving current gesture recognition. It has been proposed the focus of the gaze of the user can help the system recognize and decide the intentions of the user, as manipulations of objects on screen has shown to confuse and even mislead recognition systems. In addition to gaze tracking, the rising popularity of tablet computers has seen gesture recognition software implementation. Some leading research proposes in addition to the touch screen interaction, touch gestures can be applied as well to enrich the experience. These touch gestures make use of the imagery supplied by a gesture and the physical interaction of an on screen object to achieve a meaningful command.

One proposed method of control is through the use of gaze gestures, in this study a simple program was created to perform specific tasks based on actions observed in the user's eyes. These tasks were simple web browsing tasks. One idea proposed was the use of gaze to help the system identify and predict user intentions [35]. A similar idea to the study conducted in [35] is to use gaze gesture enhance touch gesture input of phones. One method of implementation of gaze with gestures is the system tracking the user's gaze and focus on an object on screen as a means of selection [47].

Gesture Interfaces are seeing uses in tablet computers, as such, the use of the "touch gesture" has been proposed for use with them. Taking advantage of the typical built in camera or additional peripherals that can be attached, the tablet can create shortcuts or separate commands based on the interaction performed by the user. An example is the basic touch which is the hand in the pointing posture touching an onscreen object; this simulates the basic click, or selector command. With gesture recognition, the camera can read what shape the hand is in when it 
interacts with the object and use an alternate command, such as copy, edit, etc. (common commands in tablet applications) [47].

Some studies that primarily involved camera based systems have proposed additional hardware to their systems. In many systems, a proposed addition to the camera would alert the system to broad range strokes of the hand or to activate the system when a user is in range for use. Some studies have also proposed the use of these additional systems to identify the start and end of a gesture to further increase the recognition success of these systems.

Pyro-Electric Infrared (PIR): This approach uses a 4x4 PIR sensor array to recognize hand gestures. With the use of the PIR sensor is it possible to detect motion from the user rather than motion of the camera, with this system in place, real time processing and recognition is possible. The PIR system is also able to distinguish circular movement rather than the traditional horizontal and vertical movements [10].

Motion Sensor Array: It has been noted that some design applications of gesture input require extreme precision in its design that a simple camera based system may not provide, hence the study proposes a motion capture which allows increased precision when working with the system [53].

Tool/Prop: Some hardware may be required for gesture intensive use in order to enhance gesture recognition; this includes colored gloves or labelling methods to highlight different sections of the hand. This is a novel approach to increase recognition rate at a small increase to the cost as alternate labelling do not require expensive hardware like sensors and microprocessors [24]. Some alternative approaches have been tried to minimize the cost of glove and sensors, and it has demonstrated significant improvement and recognition rates. However, when deciding an application for this technology, the cost versus performance must be considered [57]. One such example of a tool is that of a simple pencil, given that color based camera systems can track uniform objects easily, the use of an object to simulate an actual counterpart will help improve the accuracy of the system. A study was conducted to improve the means of electronic equation entry using gestures and handwriting. The main advantage of this system is that current devices such as phones, tablets and netbooks can make use of the built in camera and software rather than expensive peripherals such as dedicated pens and touch screen surfaces. This also allows the user a larger work space as they are now limited only by the 
camera's view range rather than the comparatively smaller touch screen [45]. Another approach proposed is the user using gestures to manipulate virtual objects as if they would if they had the object in hand [56]. Another utilization of smart phone technology is to use the phone itself as a tool in the recognition. As demonstrated by the large array of sensors built into the phone such as accelerometers, gyroscopes, and compass, the array of motion-sensors provides accurate and extensive data on the movement of the phone. In this article, the study was conducted using the phone as the gesturing device where in the user moves the phone in patterns that would be recognized by the system so that it may perform predetermined actions. The purpose of this approach is to minimize power overhead of other methods as the power consumption of the phone in an active state is two magnitudes higher than its sleep state. The use of efficient processing algorithms allows the sensors to use lower sampling cycles to further reduce power consumption [58].

Additional Camera: The use of the system allows for the system to recognize depth in gestures. Depth will provide the system with additional features to recognize, improving the recognition success as well as offering redundancy or in some cases, a differential error checking [44].

Sensor Device: Devices that uses the physical quantities such as position and muscle exertion allow for systems improve accuracy as data obtained from these sensors can minimize errors during gesture recognition. Devices such as the Myo Armband employ sensors such as EMG sensors, gyroscopes and accelerometers provide information such as finger positions, orientation and arm movement. This information must be calculated in image recognition and is not as reliable as sensor information [68].

\subsection{Gesture Classifications}

Gestures are typically placed into two categories, Static and Dynamic Gestures [13] [9] [1] [7]. Static Gestures are observed in an instance of a period of time, and Dynamic Gestures are observed over a period of time [7]. However, this is a simplistic approach that observes directed movement. A more in-depth approach looks at the intent in which the gestures are presented with as defined by these categories: Para-language, Body-language, and Sound-Language. Para- 
language refers to non-verbal cues the body produces such as intensity and speed of emission. Body-Language is observed as a distinct characteristic of the body being used; this can also refer to such things as pose/form and proximity. Finally, Sound-language refers to features with sound quality, an example is silence, such in the absence of sound or action can be interpreted as having meaning [12].

Typically recognition software is designed for Static or Dynamic Gestures. In the case of Static gestures, algorithms isolate the hand shape or define finger differentials [1] [5] [6]. Static Gestures are usually compared to databases for successful recognition. A sub-set of shapes that is most designed for are sign-language [3] [9] [7]. Dynamic Gestures use motion tracking to compare to a predefined database entry, however, some track the simple transition of one gesture to another [11]. Another dynamic tracking is to track motion commands such as swipes, hand rotation, etc. [4] [10].

Proposed Sign-language data models are designed similar to speak recognition software using offline and online models. Similarly, the approach to this type of recognition is based on speech recognition algorithms. As the simpler of the two types of gestures presented, the basic operation for processing these are fairly simple. The process proceeds as follows: image is identified as valid command by temporal definition or by state definition, a specific feature is extracted. The extracted feature is compared to database of predefined which will then decide what interpretation will fit the data most appropriately [3] [9] [7]. Some processing techniques commonly used for this type of recognition: K-mean [3], Markov Models [9], and Principle components analysis [7].

Some models propose a continuous active model rather than requiring an active state like the above mentioned speech recognition models. This type of model has introduced new issues into the field such as Real-Time processing, Machine Learning, and Gesture Classification [10]. This process in which this approach is bare much similarity to the process above, however, a necessary step of hand tracking is a fixed requirement. Due to the nature of the static gesture, hand tracking may not be necessary in all cases, however with dynamic gesturing, the algorithm must always be aware of the relative location of the gesture [11]. Some techniques used in this process: Hough-transform-based voting [11], K-Means clustering [11].

Touch Gestures: Using touch screen hardware installed on smart systems (phones, 
tablets, etc.) touch gestures simulates gesture interactions with objects on screen. Actions such as object selection and move are some of the most popular actions [36].

While dynamic gestures are based heavily and use similar techniques as static gestures, a distinction must be made between the two types of gestures as the uses for each type carry in of itself a meaning. In the case of static gesture, the use of this type denotes a symbol, a representation of an object in the context of the current mode of communication. The use of a static gesture is similar to pressing a key on a keyboard in that the input is of a fixed nature. Dynamic gestures are primarily used as command or action inputs. Using the same analogy as above, this is similar to the moving of a mouse peripheral. Dynamic gestures carry the notation of interaction in that the action is directed to control an object of interest presented to the user. Such an example can be seen when a child is interacting with a toy such as grabbing the object, moving it around, etc. [13]. One method examined to recognize dynamic gestures is to use hand specific gestures to establish a start and an end point for specific gestures in a continuous input. In [53], the study aimed towards building a design system for Computer-Aided Design (CAD) project using human gestures to increase the amount of information that can be transmitted to the computer. The designers decided to use static gestures to supplement the dynamic gestures for simpler recognition. The system has the user perform a static gesture with their left hand, while performing a dynamic gesture with their left. The static gesture of the left hand tells the system to analyze the actions of the right hand in order to perform the command, when the gesture of the left hand is released; the system understands that the end point of the right hand's dynamic gesture has ended [53]. One method of recognition is to instead treat all user actions as one continuous gesture where each action will be process and mapped to a related action to be performed by the computer. In the method proposed the gesture interface would not simply augment mouse and keyboard interface, but rather replace them. In this instance, the recognition method continuously reads the input and behaves in accordance to the user's actions [60].

\subsection{Gesture and Language}

Gestures are a type of language that humans create much like speech language. When using gesture language, the complexity and structure are similar to spoken language, complete with 
grammar, verbs and other such characteristics. However, much like spoken language, gestural language is learned through experience and interactions with parents or other such guardian-like figures. An example of this is the Anna experiment (1932) in which an infant was isolated from birth with minimal to no contact with another living human being. Anna was described as having "no sense of gesture" or indeed little understanding of language. However, through contact and interaction with parental figures, Anna was able to acquire language skills and develop normally much later due to rescue and care [14]. Demonstratively so, humans are known to be hardwired to develop language as even should sense such as hearing are disabled, the area of the brain that is identified as the "seat for language" remains active. Further studies find that all language learned and developed are highly structured and complex.

Children of immigrants to a new country with a different language have been documented to know language never heard before; proof that humans do not simply learn language, but part of the process of learning language is to also develop and create language [13]. In a study on aphasia it was discovered that suffers of the condition are able to retrieve words and phrases with accompanied by gestures, as demonstrated in the study, it is shown that gestures share many characteristics with speech and stimulate activities in similar areas of the brain as well [52].

In a study conducted on teacher habits in relation to gesture, it is found that culturally different background affect the use of gestures during instructional sessions. The study observed instructional sessions of mathematics to remove bias from the subject material and observed habits from multiple levels. In the early levels of instructions, teachers used similar gestural techniques to deconstruct problems, however when the sessions of advance and abstract courses, culturally different teachers used wildly different gestural techniques. Western teachers were noted to use fewer gestures and utilized less spatial gestures than eastern teachers [51].

The focus of the user's sight demonstrates the target to which their actions want to control, while this technology is mostly used as an alternative for handicapped users, it has found applications for tablet use in conjunction with other interfacing techniques. Participants performed better with Touch Based Interaction based on the study conducted in [31], which kindergarten children were examined between Gesture Based Interaction and Touch Based Interaction. It was concluded in relation to temperament, touch based interaction yielded a better 
result. In a study performed in [33] found that touch screen interaction required less focus from the user on the peripheral as well as a preference for directly interacting with an on screen object rather than through the traditional peripheral of button presses [33]. In an experiment conducted regarding touch screen task performance, it was discovered that the texture of the surface yielded different results for the task performed. The experiment concluded that different textures have specific design advantages such as a slippery surface allowing the user to perform faster movements, but at the cost of lower accuracy [46].

In a study conducted on game experiences with pens and touch surface devices, it was found that during the trials, participants did not find visual trace feedback to have importance. [24] It has been discussed that in addition to gestures being based on the internal experience of the user, gestures are also context specific unlike speech, hence its importance and close relationship to speech. It has been noted that gestures do affect speech preferences however; it is shown that gestures mainly provide demonstrative properties to the information being conveyed. On their own, gestures are more difficult to build context in relation to speech. Participants of this study had their gestures habits observed, it was found that gestures that required actions were preformed almost exclusively on the right hand, regardless of the participant's dominant hand. The study notes that this suggests that this is controlled by the left cerebral hemisphere [25]. In another study conducted on infants, similar results have been noted to have occurred; however in infants the gesture of pointing had been used instead [26].

\subsection{Designs of Gestures}

A study into hand gestures in relation to sign language has been conducted. The focus of this study was to find discomfort in the postures the language invokes. The study proposes that as this technology gains use in everyday applications, users will be in a position that requires 20 to 50 hours of use with the interface and health issues that arise with will share similarities with sign language interpreters [21]. Much like the previously discussed static gestures, the dynamic counterpart to this system is the use of simulated interactions. This system observes and interprets all movements as commands and must decide which commands will be acted upon and when. Designs for this type of system looks to minimize movement from the user to 
increase comfort when using it. As such, objects of interest are usually kept small and the range of movement minimized to reduce strain on the user [48]. In the study conducted in [55], users were asks to perform a gesture that they believe would be best suited for a specific command, such as selecting. They found that when giving commands such as positioning, selecting and activating, one finger is the norm while commands for orientation, zoom factor and deactivation, participants used the entire hand. The study also describes when manipulating the observational properties of the virtual object, participants use gestures reserved for interacting with physical objects, such as moving the volume closer to their persons for the command "zoom in" [55].

Symbol Gestures: Similar to keyboard inputs, systems like this rely on imagery invoked by gestures to issue commands and inputs. As with the issues presented before, some design considerations must be taken when creating meaningful symbols. Here are some guidelines proposed: Common/extensive use commands assigned to forms comfortable and easy to repeat, hands should be close to the body and below the shoulders, forceful impact should be avoided, wrist and forearm movement should maintain neutral forms, and fingers should avoid prolonged full extension [21] [37].

Simulated Interaction: An alternate design scheme for gestures is to simulate the user would experience if they were to interacting with a real world object. In a robotic control scheme proposed, the user would interact with an object in question while the robotic arm performs actions as controlled by the user [22].

Hand Preference for Gesture Types: In a study conducted on infants, it is found that participants preferentially use their right hands for communicative gestures [26].

The design of gesture interfaces sees a difficult issue in the balance between recognition and intuitiveness and comfort. It has been demonstrated that rigid gesture structures are more easily recognized and see a much higher success with current recognition systems, however ergonomically, these gestures are uncomfortable given long periods of continuous use; however the intuitiveness of gestures is affected by many factors both in interpretation and in origin [48] [49]. In a study performed on gestural habits of designs in a team design environment it was discovered designers use different gesture types in different roles to effectively demonstrate the theoretical design. However, a large limiting factor of a universal system of design is that gestures, by nature, draw upon the person's own personal experience, as such, ideas and physical 
meaning is lost when conveyed to another person [50]. Below are factors that influence gesture vocabulary.

1. By product of human learning process: Gestures and gesture interactions are a byproduct of the human learning process as children learn through physical interaction with surrounding objects [13].

2. Influenced Socially: As is the case with language, gestures and body language are influenced by social interactions. The effect is that different cultures and different social groups can exhibit many different changes to certain body language [14].

3. Children demonstrate innate ability of the deep structure of language and the units measuring it [13].

4. Gesture Types

1. Iconic: Pictorial gestures that represent the shape or style of the object being referred to [25].

2. Metaphoric: Pictorial gestures that represent an abstract concept being referred to [25].

3. Deictic: Pointing gestures that refer to a referent of the environment [25].

4. Beat: Rhythmic movement of the hand(s) that demonstrate the discourse that occurs [25].

5. Speech difference between gestural or non-gestural: In a study conducted in relation to speech patterns and gestures, it was found that participants that were not allowed to use hand gestures used much more descriptive words and metaphors when asked to describe line drawings. However the study also notes that with the elimination of gestures, there was an increase in spatial description while there was a decrease in demonstrative phrases [27].

6. Touch: An experiment conducted on children of advanced age concluded that TouchBased interactions showed higher learning performances to pure Gesture-Based interactions. It was observed that the Touch Based Interface (TBI) offered more stimuli to the participants resulting in better results than the Gesture Based Interface (GBI) group [31]. 
While the user of spoken language can be unique and completely foreign to different cultures, there is cross-cultural vocabulary in gestures. The most basic and simple of these is the deictic gesture, it was shown that users of different languages can use deictic gestures to establish links in another culture's language; this enhances the learning experiences as this allows a culture to learn the similarities between the two languages. Deictic gestures, while simple and basic, conveys a large amount of information, and it is because of this simple nature that it is an effective tool in gesture-learning systems [51].

\subsection{Summary}

In the background study various recognition methods were investigated and examined. In the various algorithms and technology, there is very little to no consideration in the design of gesture characteristics, ergonomics and recognisability. Interestingly, the approach only considers varied and shapes that can detail and demonstrate recognition success of the algorithm. Furthermore, in the study conducted in, it was found that sign language interpreters suffer from hand injuries and disorders from the practice, indicating that design foresight into the prolonged usability of the language may not have been considered, or that greater emphasis was placed on recognisability over the comfort of the user [21].

It is therefore important that a method of identifying and observing characteristics of good gesture design and poor gesture design should be created and implemented to accomplish this. As identified in [21], exertion by the hands in unhealthy positions and muscle strain is the cause of such injuries, some focus and investigation into this will illuminate characteristics of gestures and hand positions that would cause such injuries.

Some technologies identified to aid in this study are sensor devices, specifically the Myo Armband. Given its simple nature of operation and accessible data registers, this device can be employed to investigate exertion on the user [68]. 


\section{Chapter 3}

\section{Gesture Design Investigation Methodology}

This chapter will detail examination of current gesture databases through different aspects. The approach will look at the following: the types of gestures used in these databases, what kind of static gestures are used, what kinds of dynamic gestures are used, and the ergonomic design of the gestures. From this examination, a set of guidelines should be produced to ensure new gesture designs meet the requirements of being easy to recognize, intuitive, and comfortable.

Gesture types are important in this study as these are often defined by the user's culture, background, and experiences [51]. Current studies use touch interfaces as a point of reference for the types of gestures developed, as such, most databases are intuitively adjusted with users that are already familiar with touch technology or have some level of experience with the gestures and actions associated with its use. Databases from different countries and cultures will also demonstrate similarities in some gesture types, as well as highlight key differences that will be useful in gestural designs. To effectively achieve this, all gestures will be placed into three categories: Iconic, Metaphoric, and Deictic [50] [51] [55].

Static and Dynamic gestures can carry different meaning and have different recognition methods, as well as varying challenges associated with each. Static gestures are normally shown 
to be iconic in nature and are meant as a substitute as a representation of an object or concept. The commands that derive from this set of gestures closely resemble that of button presses. Static gestures invoke a fixed command each time it is presented. Dynamic gestures are gestures that are normally shown in metaphoric or iconic in nature. These gestures are closely related to actions and direct interactions. This type of gesture usually produces commands that closely resemble mouse movements. Dynamic gestures invoke involvement and actions within the environment the user interactions with the system is in. Systems typically recognize dynamic gestures as static gestures with respect to the time-domain, as distinction must be made between the two gestures [50].

Another consideration is the ergonomic design of the gesture. A failure of systems that use touch gestures is that the design does not enhance the experience of using the system, a replacement of current user methods should be for the simple purpose of novelty but rather it fulfills a purpose of either increasing efficiency, or that it is more comfortable for the user. Gestures that strain the user will limit the active time the user is able to use the system, as such gestures should be relaxed and provide the least amount of strain to the user rather than the distinctiveness of the gesture. To accomplish this, the use of the Myo armband will be employed. The Myo armband is a device that uses EMG sensors to measure activity in the forearm to determine the shape and form of the hand. This allows the device to recognize what gesture the user is making. Using this tool, it is possible to record and display the effort and strain the user undergoes for each gesture [49] [51].

As a new technology that is rapidly gaining popularity, there are little considerations in the design of the gestures to be used. The best references for these designs are touch surfaces that are still relatively young as well. As such, the failings and shortcomings of these designs are affecting the willingness of users to experience the upcoming technology. It is also a fault of developers that do not put thought or importance of interface design when designing the interactive system as the experience of the interaction affects a user's willingness to continue using the interface. However, as the technology continues to advance, new status quo, cross cultural elements, and customs emerge, the technology and the designers are incorporating these new trends into their databases, however a guideline should still be set on how this approach should be taken [49]. 


\subsection{Gesture Types}

Recognition of gestures can be categorized into three types: Iconic, Metaphoric and Deictic. Gestures taken from databases will be categorized in to these three categories. Each type of gesture serves a specific purpose.

Iconic gestures are the most simple and most used gestures for recognition, these are primarily static gestures that rely on the image they invoke to convey their meanings, this plays favorably to recognition software as the images they invoke are either commonly experienced symbols, imagery, or objects, the user may use gestures to simulate or represent the functionality of an object that may or may not be on screen. American Sign Language is usually used as iconic gestures; Figure 3.1 demonstrates the American Sign Language (ASL) equivalent of the letters "A", "S", "L". Dynamic gestures can also fall into this category as they invoke an image or symbol. To fall into this category, the gesture must display these characteristics: they are symbolic, either using an abstract shape that is corresponding to a fixed meaning. They are in the general shape of an object in question. They are a simulation or representation of an action that an object in question can perform [21] [25] [37].

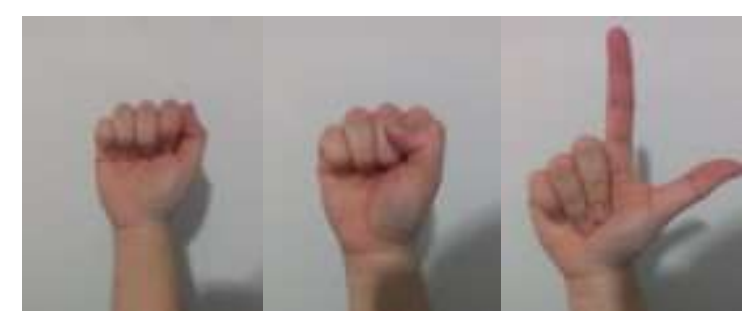

Figure 3.1: Example of iconic gestures, American Sign Language letters ASL

Metaphoric gestures are primarily dynamic gestures that invoke abstract functions or concepts. Gestures that typically fall into this category are movement gestures, such as the act of picking up a virtual object and moving it around the interactive environment. Figure 3.2 shows an example of a metaphoric gesture. Other abstract interactions will also fall into this category such as describing the shape of action of an object. To fall into this category the gesture must 
display the following characteristics: They do not correspond with any fixed meaning. There is no real world object that corresponds with the actions performed in the gesture [25] [50].

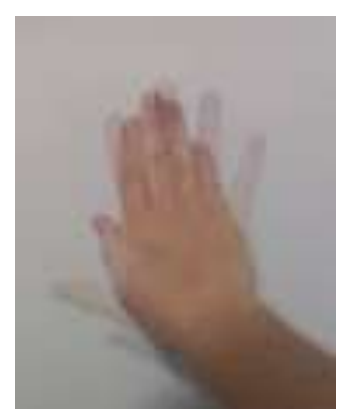

Figure 3.2: Example of Metaphoric gesture, the spreading of the hand

Deictic gestures as shown in Figure 3.3 are gestures that point to a direction, these types of gestures of closely related to iconic gestures; however, deictic gestures are spatial in nature and require awareness of the space they are used in to be effectively utilized. Both dynamic and static gestures make use of this type. The main gesture that falls into this category is the pointing gesture. To fall into this category, the gesture must display the following characteristics. Any gesture that has a directional vector as part of its information is considered a deictic gesture. This type of gesture draws attention to a specific location for the purpose of conveying information. Directional movement as part of a simulation or representation is not included in this category [25] [50].

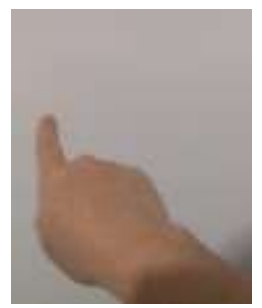

Figure 3.3: Example of deictic gesture, the pointing gesture 


\subsubsection{Static Gestures}

Static gestures as shown in Figure 3.4 are gestures that issue a command specific to the shape that the system interprets. Sign language, key prompts, and key commands are commands that make the most use out of static gestures. Given their simple nature, static gestures have a higher recognition success rate given that highly unique gestures can be used to issue commands to a system that uses few commands.

Databases that tracks these gestures are mostly image based processing, as such; image pattern recognition is the most widely studied method for tracking and recognition. However the limitations of this approach are apparent as the system only achieves success when the hand shapes are highly varied from one another. It is hence proposed that modeling techniques also be applied to these system to improve upon the success and to allow the system to recognize more subtle changes and variations in the user's hand shapes. This allows for a larger gesture set that the system will be capable of recognizing [1] [3] [7].

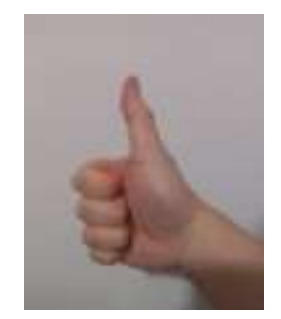

Figure 3.4: An example of a static gesture

Currently the most effective use for static gestures is the emulation of a button press, or in this instance, an iconic use. Databases that use static gestures map the shape and profile to a set command, most often a key press, like in the American Sign Language, the shape of a specific gesture corresponds with a specific letter. Other examples include making a fist to command the system that a "right-click" command is being issued. In design system aimed to making gesture interaction a design tool similar to auto-cad, static gestures are used to select tools, often by imitating their shape to switch use to these tools. This allows the system to obtain 
information from the user based on experience rather than memorization of names and symbols as the user can convey the information visually using shapes that are much more memorable to the user than names.

\subsubsection{Dynamic Gestures}

Dynamic gestures, shown in Figure 3.5, are gestures that generate a motion corresponding to a command, the gesture, from its direction, hand shape, and overall speed can convey a large amount of information for the system to interpret. They are often used with commands such as object moving, control, and interaction. There are currently no standard method of recognizing dynamic gestures, however some issues that are still currently with in studies is how to define the start and end of a gesture as inaction or stillness of a dynamic gesture can have meaning as well.

Databases that use this system often store the information in frames, each frame is treated as an individual image and they are transformed into simplified representations and compared to a stored value in the database, if the system finds a match, then the system will use the corresponding movement to match with the appropriate command. However, newer algorithms use sub-modeling techniques to gesture movement, allowing the system an alternate method to recognize dynamic gestures [7] [11].

Figure 3.5: Example of dynamic gesture, wave out

Dynamic gestures see many use in object focused applications. Currently databases that have their primary focus on dynamic gestures often use gestures in a simulated environment. 
One such application is gaming where the user will interact with objects on screen as they would if the object in question were real. The use of this can be both Iconic and Metaphoric; the object in question can be a representation of a real object, demonstrating Iconic use. The user is showing to the system the actions associated with the object. However, metaphoric approaches see the dynamic gestures controlling more abstract concepts, like interacting with a representation of a concept with no physical equivalent [12].

\subsection{Gesture Designs}

\subsubsection{Exertion}

The exertion of a gesture impacts the usefulness of the system; exertion in this context is defined as the user exerting their bodies beyond a reasonable and comfortable degree. An example of an exertion gesture is a pointing gesture with the finger fully extended; repeated use of this gesture is known to incite pain in the subject. Exertion and the effort required to make the gesture are factors in the comfort and usability of the system. Current databases will be analyzed and observations will be made to how much exertion the gesture places on the user.

Using the gestures extracted from databases, EMG readings will be recorded in reference to the signal levels required. The measurement of the muscle group in the forearms will give an indication to the level of exertion the user experiences when making a gesture. To accomplish this, a Myo armband was used to measure the muscle activity when the user makes a specific gesture. The subject used in this measurement is a male in their mid-twenties.

The Myo armband is a device that uses EMG readings to map gestures, by matching the readings to a predefined record, a gesture can be recognized. Each armband has 8 sensor modules called pods that attach to position in the arm in order to get readings. The pod numbering in accordance at the time of experimentation is shown below [68]. 


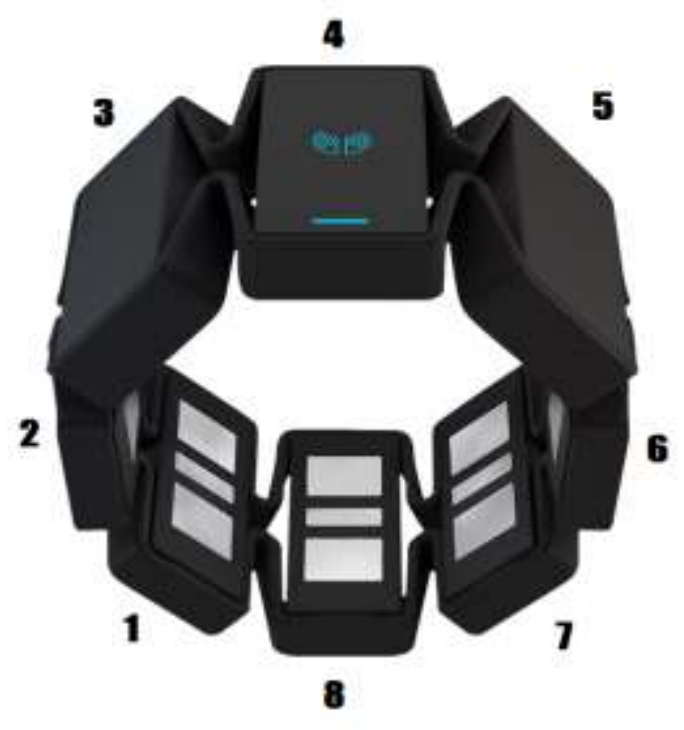

Figure 3.6: Myo Armband pod numbering

As the measurement device is an armband with sensors, below is a description of the pods and their relative locations to the muscles of the arms [69].

- Pod 1: Flexor Carpi Ulnaris

- Pod 2: Brachioradialis

- Pod 3: Extensor Carpi Radialis Longus Brevis

- Pod 4: Extensor Carpi Ulnaris

- Pod 5: Extensor Digitorum

- Pod 6: Extensor Carpi Radialis Brevis

- Pod 7: Flexor Carpi Radialis

- Pod 8: Flexor Carpi Ulnaris

The Myo was selected as the device to measure exertion due to its ease of use and simple design. The Myo device offers easy access to the EMG signals of different subjects. It should be noted that due to the varying characteristics of different people, EMG signals, even with precision instruments will still vary from person to person. Thus, the Myo Armband is not in an immediate disadvantaged to more precise instruments. Additionally, the Myo Armband was 
designed with various tools to access the data registry of the device to create custom software to make use of the data obtained by the device. This made the Myo Armband an excellent candidate to measure exertion levels once the appropriate method had been identified.

Once the Myo Armband has been secured on the arm, the subject entered a rest posture and waited until the Myo Sensors had reached a steady state. Once a steady state had been achieved, a button was pressed to start the averaging program; the gesture was then performed during this time. For static gestures the position is held until a steady state was achieved, for static gestures a pilot study was conducted to determine the repeatability of the measures. This pilot study was performed on ASL gestures A, B and C (Appendix 1, Table 4.2). In this pilot study each of the three gestures was held for an average of 2 seconds each and the steady state values were recorded from the Myo output. This process was repeated 3 times with a rest period in between each trial. The 3 measures were averaged; this process was repeated for the other 2 gestures. It was found that the 3 values obtained for each gesture within $+/-10$ activities levels. Therefore it was decided to conduct one trial per gesture, the static gestures were held for 2 seconds each. Dynamic gestures were performed 3 times in succession, and the 3 values obtained from the Myo device was averages for each gesture. Once the actions previously have been performed, a button is pressed to stop the program and begin its calculations. The values are then recorded into a data sheet. The subject enters a period of rest and prepares the next gesture. This process will be repeated until all database gestures have been recorded.

As an initial test of the Myo armband, the arm was made into a tight fist (Figure 3.10) in order to get large sensor readings, as the graph indicates the sensors have a range of -127 to 127 , which will be used in this study to simplify the examination of the exertion on the user. Figure 3.8 is a graphical representation of the data obtained by the Myo device. Many samples were taken using a tight fist and it was found that the highest appropriate exertion level the user experiences is 110 to -110 . Currently the developer of the Myo Armband has not released full data specification on the sensors of the device, and has described the values as activation values of the sensors [68]. During a test with the rest position it was found that the rest position (Figure 3.9) when the hand is fully rested for a period of time, that value read on the graph was close to 0 , though features of signals were still apparent. For the purposes of this study, the rest position and all values close to that feature will be considered 0. Figure 3.7 demonstrates the graphs 
produced using measuring software provided by the developer; the gesture used was a tight fist as demonstration of the possible exertion a user can experience.
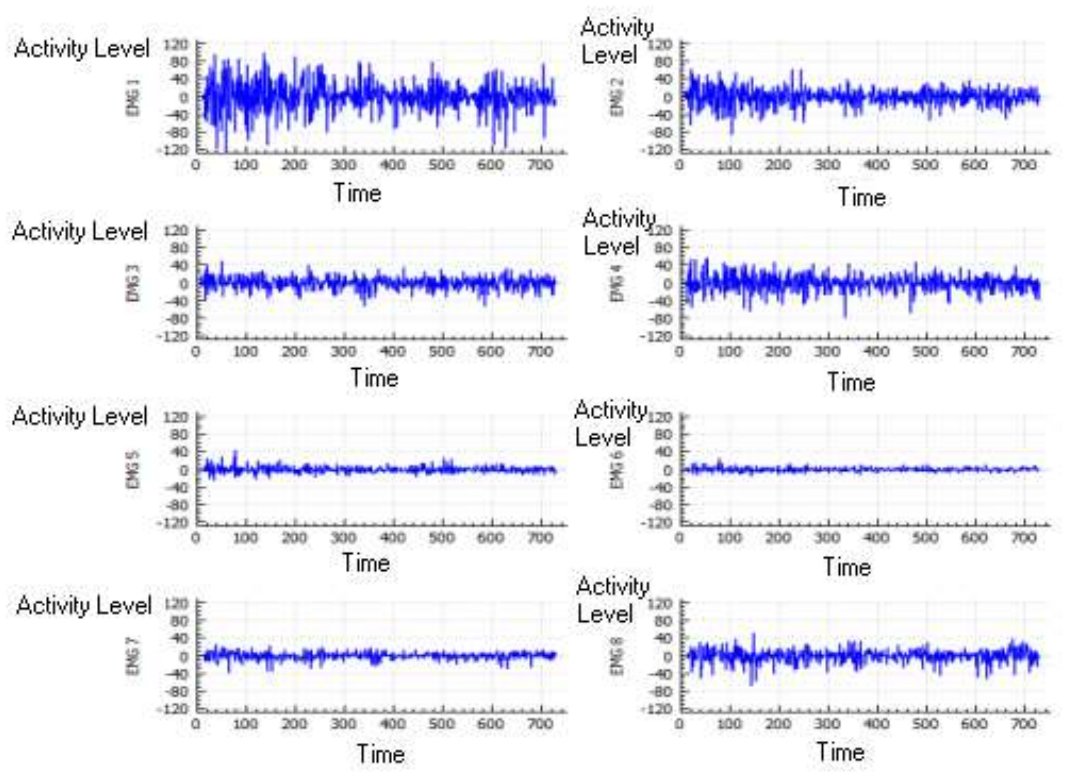

Figure 3.7: Myo EMG reading of a closed fist

First a neutral stage will be established as a reference point for the rest state, any activity that deviates from this state will be considered exertion on the user. The user's arm will be relaxed and held idle, facing a computer camera as the standby phase of the system. Following a list of gestures found previously, the measurements of the exertion on the muscles will be recorded and compared to the rest state and a modified relaxed gesture to demonstrate reduced exertion. Gestures used for the same or similar commands will also be compared to find the gesture with the least exertion.

In the context of this study, the activation levels (bits) of the sensor data will be referred to as the Activity Level as greater exertion is indicated by higher activity in the muscle groups. Common features will be observed between the gestures with the least exertion to establish guidelines into the design of gestures for recognition. Table 3.1 is a chart representing the hand in its neutral or rest state. The chart will be simplified as a range from 0 to 110 , where 0 is the rest position and the user fully exerting their hand will be 110 . These values were obtained by 
averaging rectified signal. The values were then filtered to the nearest tenth to remove noise. Equation 3.1 was implemented into the algorithm in Figure 3.8 which demonstrates the simple algorithm used to accomplish this. In Equation 3.1, A is the average Activity Level, D is the data from the pod, $\mathrm{P}$ is the pod number, and $\mathrm{N}$ is the number of samples the device took during the gesture recording process. Even in the rest position, the subject still gives off minor readings, however these readings are still the lowest given any activity will produce a larger signal. During a trial with the subject, it was found that the highest value produced from full exertion was 110; hence this value will be used as the upper limit of the readings. Table 3.2 has the Activity Level normalized to 110, which was the highest value obtained during the recording process.

\begin{tabular}{|l|r|r|r|r|r|r|r||r|}
\hline Gesture & Pod 1 & Pod 2 & Pod 3 & Pod 4 & Pod 5 & Pod 6 & Pod 7 & Pod 8 \\
\hline Rest & 0 & 0 & 0 & 0 & 0 & 0 & 0 & 0 \\
\hline Fist & 110 & 70 & 40 & 80 & 40 & 20 & 40 & 60 \\
\hline
\end{tabular}

Table 3.1: Rest and Exertion point reference table (Activation Bits)

To create a better comparison for other subjects, Table 3.2 is the normalized activation level of Table 3.1.

\begin{tabular}{|l|c|c|c|c|c|c|c|c|}
\hline Gesture & Pod 1 & Pod 2 & Pod 3 & Pod 4 & Pod 5 & Pod 6 & Pod 7 & Pod 8 \\
\hline Rest & 0.00 & 0.00 & 0.00 & 0.00 & 0.00 & 0.00 & 0.00 & 0.00 \\
\hline Fist & 1.00 & 0.64 & 0.36 & 0.73 & 0.36 & 0.18 & 0.36 & 0.55 \\
\hline
\end{tabular}

Table 3.2: Rest and Exertion point reference table (Normalized)

As these are the muscles responsible for finger movement, the usage of these muscles has a direct correlation with how much exertion will be placed on the user. Hence the higher the uses, as measured as an EMG reading, the more exertion it will be on the user. In trials with the device balling the hand into as tight fist as produced the highest reading of any of the identified gestures, this is shown in Figure 3.10. 


$$
A_{P}=\frac{\sum_{n}^{n=0 \rightarrow N}\left|D_{P}\right|}{n}
$$

The algorithm in Figure 3.8 was implemented as a driver for the Myo device.

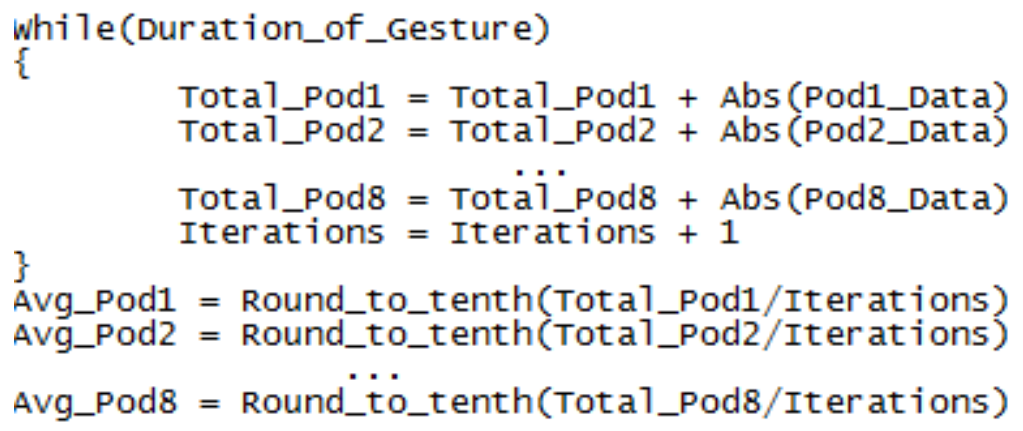

Figure 3.8: Sensor Data Rectifying, Rounding, Averaging Algorithm

\subsubsection{Position}

Another factor in the ergonomic design of a gesture is the range of movement that the user must make to produce an approved gesture. Gestures that use the upper limits of this range causes discomfort and pain in some instances, even without prolonged use. An example of an extreme form of gesture is a "stretch" gesture, illustrated in Figure 3.11, where the natural movement of the wrist or finger is flexed to the absolute limit that the user can tolerate without injury. Extreme gestures such as these will cause injury and damage over time and repeated use. The range of movements possible could go beyond that the natural unassisted range, an example is the use of the other hand to flex a finger past what the muscles and tendons are naturally capable of [49]. 


\section{Figure 3.9: Relaxed hand used in experiments (Reference $0,90^{\circ}$ )}

To establish the range of movement of a gesture the reference point of a hand in rest position is taken. The two extremes from this rest position are the full extension and spread of the fingers to a tightly clenched fist. The reference point will be considered 0 and the two extremes will be assigned a value of 10 and -10, where the value of 10 will be the fingers fully extended and spread out and -10 will be the hand in a clenched fist. The values will be assigned to each finger and the thumb in order to observe the movement range of the gestures and its effect on the user. The fingers were measured where a fully extended hand measured 0 degrees and a fully clenched hand will have the fingers pointing the opposite direction, or at the 180 degree mark with a relaxed hand approaching approximately 90 degrees. Due to the inconsistent nature of gesture making and for comparison using the user's limits, the system described above was implemented instead. In a test with using measurements it was found that for more difficult gestures, measuring gestures became inconsistent due to fatigue, difficulty maintaining the gesture or involuntary muscle twitches. The scale was introduced to reduce the time required to sustain the gesture. With degree measurements it was found that the subject experienced an error of +/- 10 degrees roughly, however with the scale there was little to no error, and gestures fell within their expected range $95 \%$ of the time.

Figure 3.10: Closed fist used in experiments (Reference $-10,180^{0}$ ) 
Due to the extra movement of the thumb in reference to the fingers, additional values have been assigned to observe and measure gesture exertion. The thumb in addition to flex and extend movement is also capable of vertical and horizontal movements, this movement will be also assigned a value of -10 to 10 .

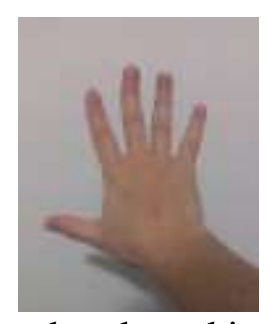

Figure 3.11: Fully extended open hand used in experiments (Reference 10, $0^{0}$ )

Below is a chart demonstrating the breakdown of how movement range is represented. Each gesture will be identified and have its corresponding movement range recorded.

\begin{tabular}{|l||c|c|c|c||c|c|c|}
\hline Gesture & Thumb Axis & Thumb Flex & Index & Middle & Ring & Little & Wrist \\
\hline Rest & 0 & 0 & 0 & 0 & 0 & 0 & 0 \\
\hline Fist & -10 & -10 & -10 & -10 & -10 & -10 & 0 \\
\hline Extended & 10 & 10 & 10 & 10 & 10 & 10 & 0 \\
\hline
\end{tabular}

Table 3.3: Rest, flex and clenched reference

The choice using a scaling system of -10 to 10 is due to different persons has different physical characteristics. For example the subject in the experiments has a range of movement of 0 degrees with respect with the palm; however, another person had measured to have a maximum flex of 5 degrees. The scaling was implemented using the subject's maximum limits, and the use of this system will enable other studies into this interaction to use a similar system for comparison. Table 3.4 demonstrates the flex indicator as degrees where the top row values are the range as defined in this study, and the bottom row values are the corresponding degrees. 


\begin{tabular}{|c|c|c|c|c|c|c|c|c|c|c|c|c|c|c|c|c|c|c|c|c|c|}
\hline Scale & 10 & 9 & 8 & 7 & 6 & 5 & 4 & 3 & 2 & 1 & 0 & -1 & -2 & -3 & -4 & -5 & -6 & -7 & -8 & -9 & -10 \\
\hline Degree( $\left.{ }^{0}\right)$ & 0 & 9 & 18 & 27 & 36 & 45 & 56 & 63 & 72 & 81 & 90 & 99 & 108 & 117 & 126 & 135 & 144 & 153 & 162 & 171 & 180 \\
\hline
\end{tabular}

Table 3.4: Range assigned to each value by degrees $\left(\mathrm{X}^{0}\right)$

Figure 3.13 demonstrates the process in which the hand positions will be recorded for static (A) and dynamic gestures (B). The subject will first perform the gesture, if the gesture was static, the subject will hold the gesture while each individual finger's position is recorded using the scale, once the fingers have been recorded, the thumb's bend and angle are recorded. Once the thumb has been recorded, the wrist bend is recorded. For dynamic gestures, the entire gesture is performed, in this process the maximum range that the gesture has achieved is measured as the hand is held in a position for measuring. Once again, the fingers will be measured first, then the thumb, and then the wrist. The data is then recorded and the subject prepares the next gesture. Table 3.4 shows the angle range for each reading of the scale.

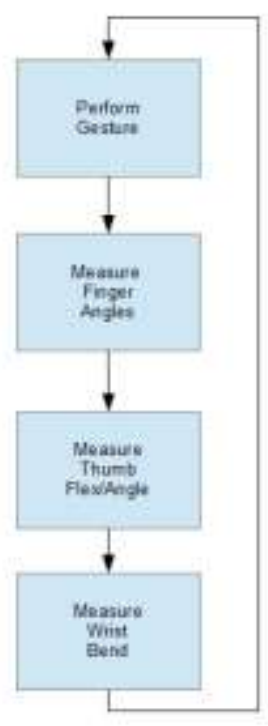

A

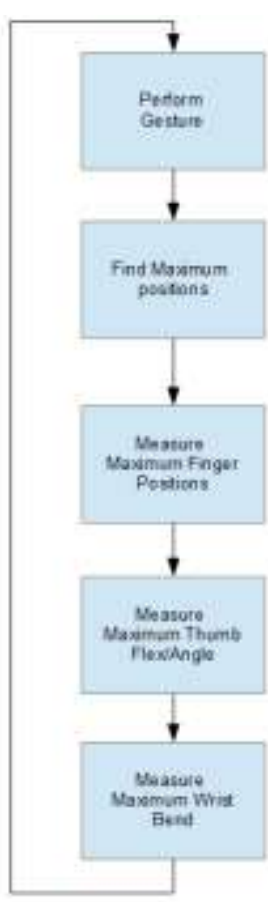

B

Figure 3.12: Position Measuring Block Diagram; (A) Static, (B) Dynamic 


\subsection{Summary}

With this method of classifying gestures into sub categories and investigating their effects on the user, a better understanding of how gesture interaction with machines can be achieved effectively. The consideration of the type of gesture to be placed into the system will help design its look. Iconic, metaphoric, and deictic gestures each have separate meanings and frequency of interaction with the user on a regular basis, the categorization of gestures in this style enable the designer to select a gesture that maximizes recognition and minimizes exertion on the user. Furthermore, the separation of static and dynamic gestures allows designs to design their focus on how their system will respond to these two types of commands. It has been shown that the algorithms for both dynamic and static gestures can vary greatly, and if the system is able to recognize which type the user is attempting to make, can select the more appropriate recognition algorithm.

Another focus of gesture interaction with a computing system is the exertion on the user, if the system causes more exertion on the user than traditional systems, then the use of gesture recognition in the system would be useless. Consideration must be made such that the minimization of exertion and effort for the system to be effective. The assessment of currently developed gestures will help in reducing exertion on the user given that modification or alternate gestures can be used in place to reduce exertion. This focus needs to be applied to movement range of the hand as well.

This approach to categorizing and observing will allow for a better understanding of how gesture interaction with machines should be approached. The use of gesture types allows the system to place more emphasis on a specific type of gesture given its specific and unique application. Furthermore, while the use of static gesture is a more simple approach, the nature of how humans communicate using a combination of static and dynamic gesture should be reflected in how machines are developed to recognize this. As the relation between human and machine interaction is further deepened and explored, it is also important to observe the effects the interaction has on the user, in this instance, exertion and comfort will define the success of the system to a growing user base. 


\section{Chapter 4}

\section{Experiments and Results}

As outlined previously gestures were obtained from databases currently in use for gesture recognition development, these were categorized into different gesture organizations and gesture types. They were then examined in the aspects of exertion and movement range.

For the gesture classification, gestures were individually looked at and counted in databases and compiled. Gestures that were the same or similar were disregarded for subsequent inclusions, some factors into separating gestures with similar shapes and movements are the meaning and purpose implied in the original context where the gesture is used. For example it was found that the fingers fully extended and closed was a common gesture in all databases; however its orientation altered the meaning of the gesture or the information it conveyed. These modifications were considered different gestures and classified accordingly. The classification noted that gestures used tend to be approximations to hand gestures used when interacting with real world objects, such as dial and lever controls.

The exertion of the gestures were also measured using the Myo Armband, as shown, the greater the exertion by the user, the higher the EMG readings are on picked up by the armband. Gestures compiled in the classifications previously were all subject to stress measurement using the Armband, with the EMG levels recorded into a table. The records indicate what the readings were of each pod, or sensory section of the device. It should be noted that maximum flex or a tight fist do not necessarily produce the highest readings as the user may not be under full 
exertion when making the gesture. As the figures demonstrates that pod 3 and 4 experience the most exertion, in reference to the placement of the pods on the subject these two sensors correspond with the Extensor Carpi Radialis Longus Brevis muscle and the Extensor Carpi Ulnaris muscles with the values 44.7 and 39.5.

The movement ranges of the gestures were also considered with respect to the fingers, thumb and wrist. As with the previous experiment, the categorized gestures were measured in accordance to the tolerances and limits of the subject. As shown in the previous experiment, the maximum extension of the fingers and thumb as well as making a fist both require exertion and the resting point is in the approximate middle location between the two extremes, the scaling was decided to be -10 to 10 where -10 represented the fingers positions are touching the palm, and 10 representing the fingers fully extended. The thumb demonstrates a wider range of movements and thus was given two measurement parameters in the thumb axis and flex. These were also assigned a value of -10 to 10 . The wrist, as the final component in this study is assigned a value between 0 and 10, where 10 is the maximum flex it can make in any direction. In this study it was found that gestures used in current databases have a tendency to induce maximum flex or maximum retraction in combinations to achieve their intended gestures. As such, many of the gestures have a rating of 10 or -10 for their respective fingers and wrists.

The trial was conducted in computer lab with the participant sitting down in front of a desktop computer with data capture software to capture and process the data stream from the device. The participant has the Myo armband secured on their right forearm. To establish reference points for the purpose of this study, the subject made an effort to obtain full exertion by as many muscle groups as possible, once full exertion as recorded, the subject entered a period of rest state to obtain the rest readings. The process begins again with the subject performing gestures from the compiled database in trials of three; the data is averaged between the three trials for each gesture and recorded. This process is repeated for all gestures listed in the database.

\subsection{Gesture Categorization}

As previously discussed, gestures were classified into Static and Dynamic gestures and then 
further categorized into Iconic, Metaphoric, and Deictic types. In the databases used in this study, a total of 66 gestures were identified and categorized. Table 4.1 demonstrates this categorization. This table was compiled using databases taken from various recognition studies as well as independent research databases obtained on-line; research databases comprised of multiple participants with both genders and a variety of skin tones.

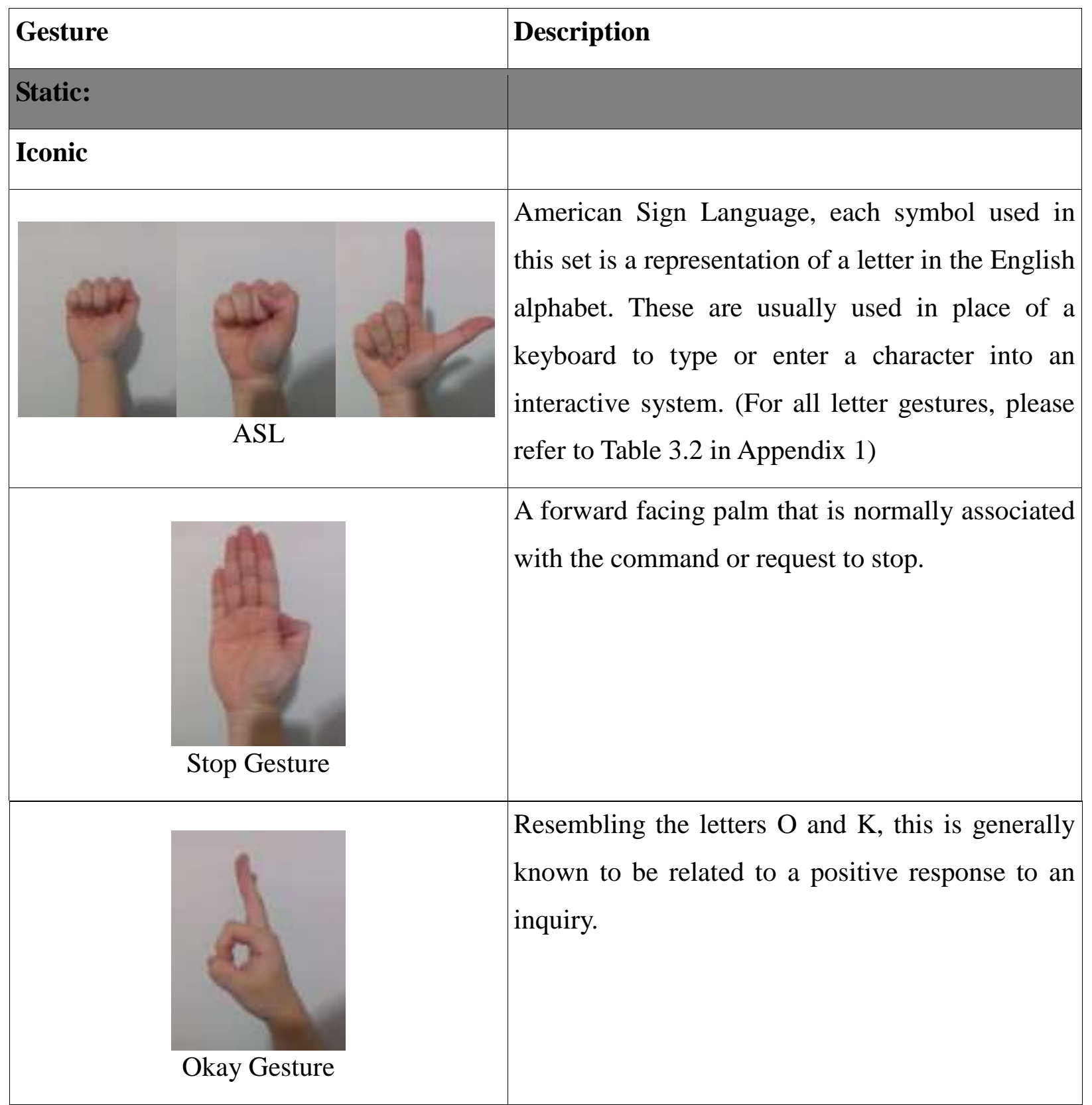




\begin{tabular}{|l|l|}
\hline Fingers Closed & $\begin{array}{l}\text { The fingers are closed together simulating a flat } \\
\text { surface or a floor. This gesture is sometimes } \\
\text { accompanied by another gesture made by the } \\
\text { other hand representing another object on this } \\
\text { surface. }\end{array}$ \\
\hline Thumbs up & $\begin{array}{l}\text { The Fingers are balled up into a fist with the } \\
\text { thumb facing up, this is associated with the } \\
\text { concept of "Yes" in an inquiry }\end{array}$ \\
\hline Point & $\begin{array}{l}\text { The Fingers are balled up into a fist with the } \\
\text { thumb facing down, this is associated with the } \\
\text { concept of "No" in an inquiry }\end{array}$ \\
\hline Deictic & $\begin{array}{l}\text { Tatabases that require spatial awareness as part } \\
\text { of its interaction. } \\
\text { deictic gesture and is used by the majority of } \\
\text { anterest by the user, this is the most common }\end{array}$ \\
\hline A single finger point into the direction of \\
\hline
\end{tabular}




\begin{tabular}{|l|l|}
\hline Hand point & $\begin{array}{l}\text { Using all fingers and thumbs to point to specific } \\
\text { direction, this is a highly uncommon pointing } \\
\text { gestures but it is sometimes used when confirming } \\
\text { four or more objects are related to the direction } \\
\text { identified. }\end{array}$ \\
\hline Two-Finger Point & $\begin{array}{l}\text { Using two fingers to point in specific direction, } \\
\text { this deictic gesture is normally interchangeable } \\
\text { with the single point gesture; however, sometimes } \\
\text { it is used to declare two objects in relation to the } \\
\text { identified direction. }\end{array}$ \\
\hline Iconic & $\begin{array}{l}\text { The hand is in a neutral position and makes a } \\
\text { circular motion right at the wrist. This simulates } \\
\text { an object rotating right. }\end{array}$ \\
\hline Circular Right & $\begin{array}{l}\text { The hand is in a neutral position and makes a } \\
\text { object rotating left. }\end{array}$ \\
\hline
\end{tabular}




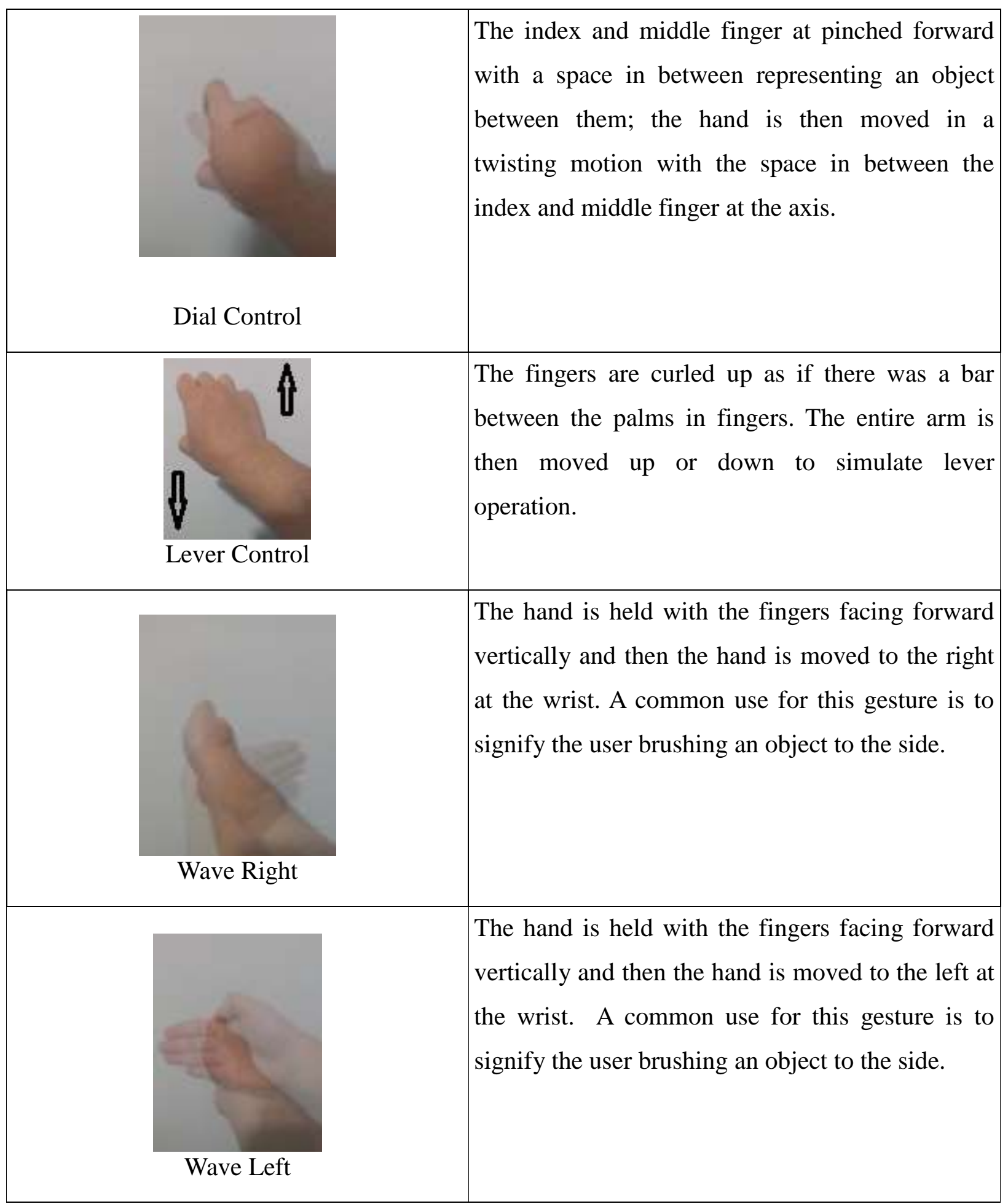




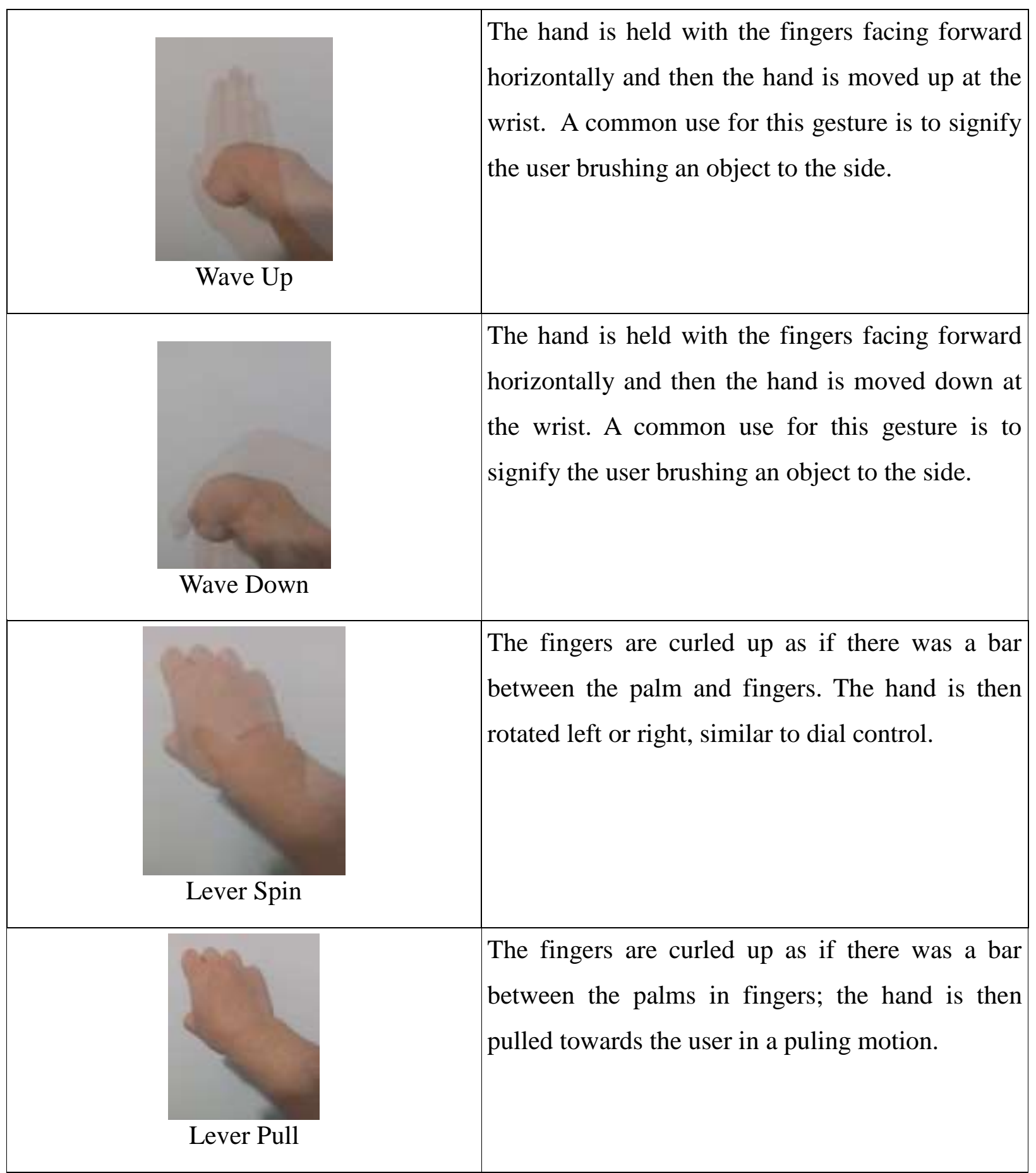




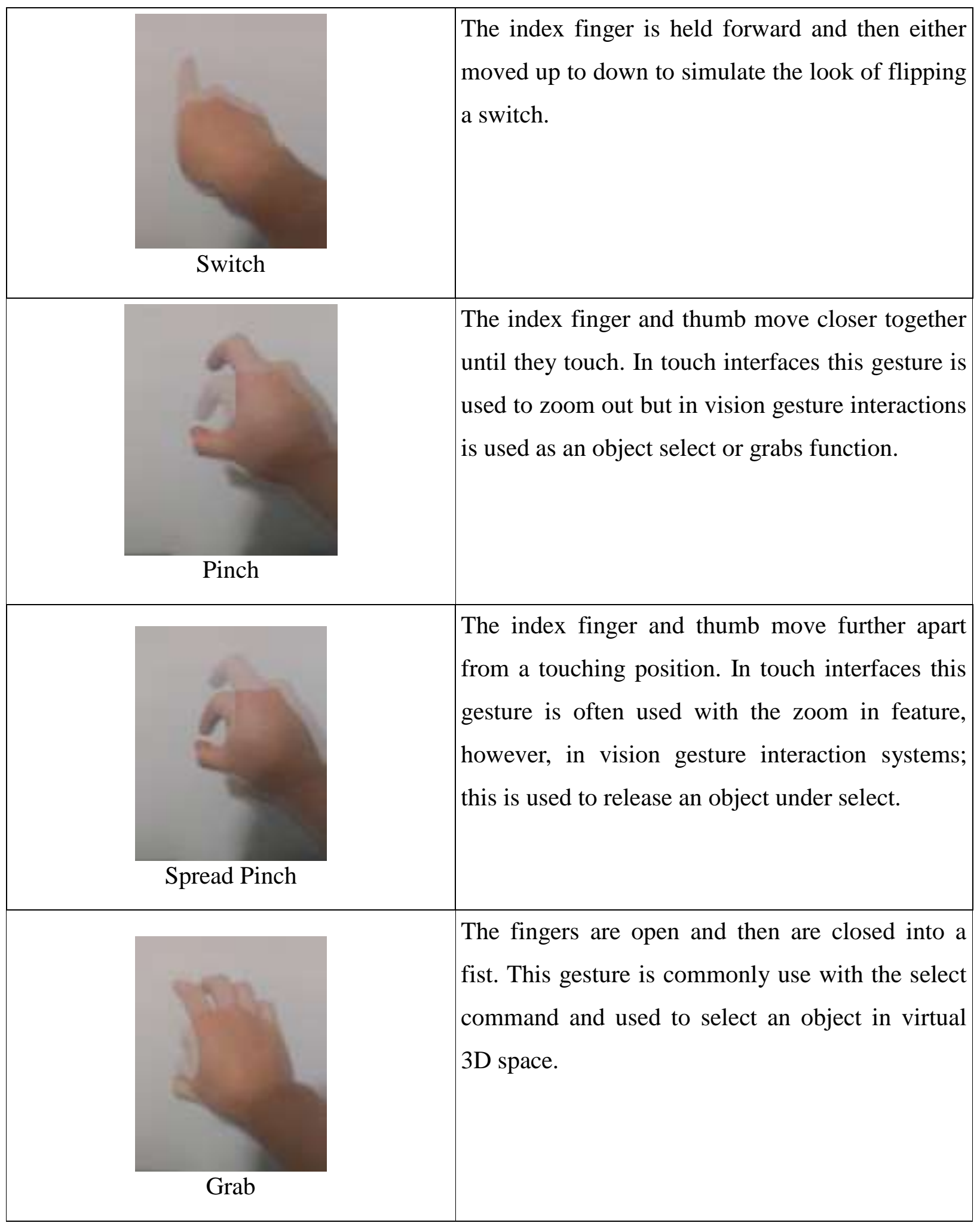




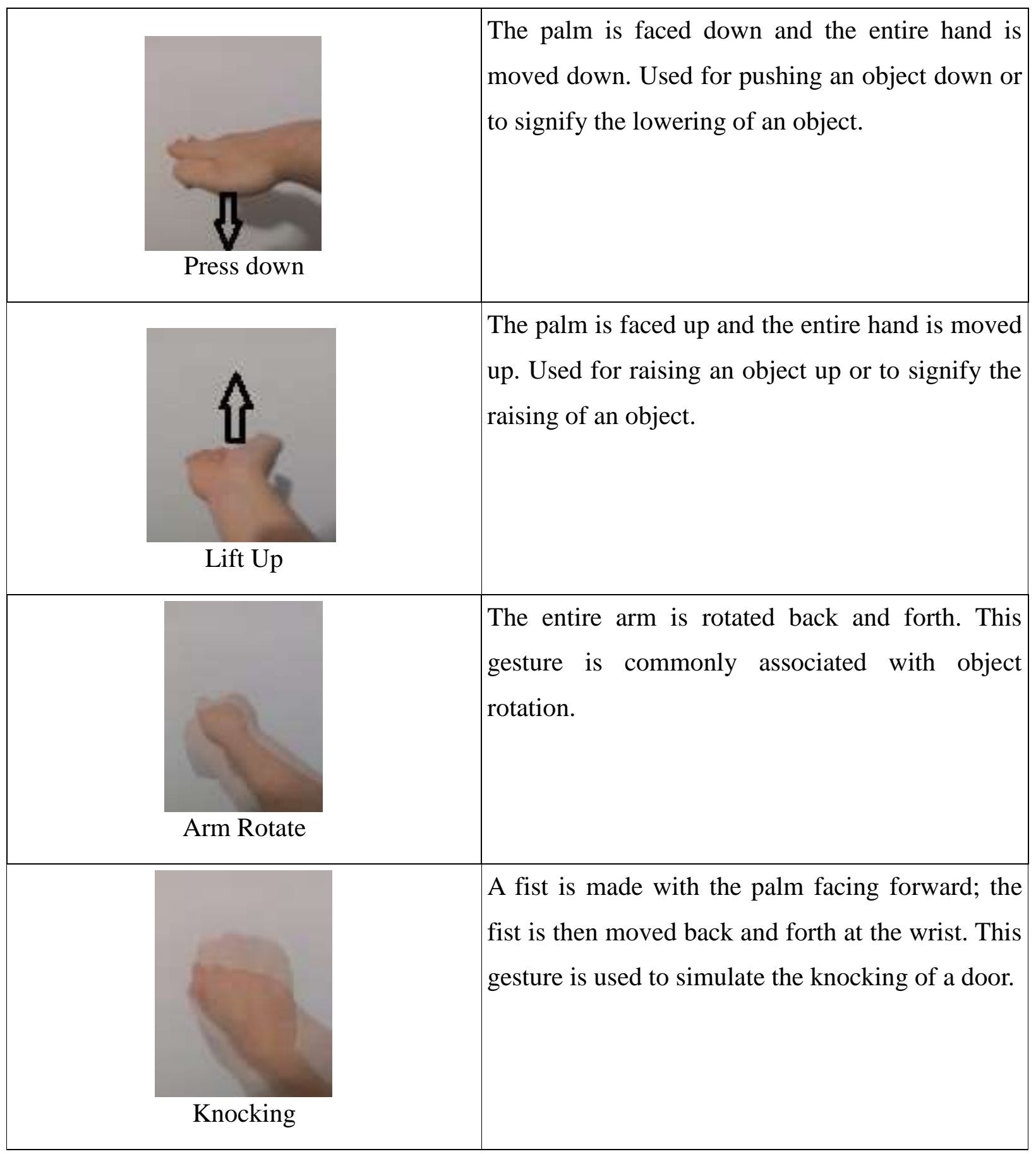




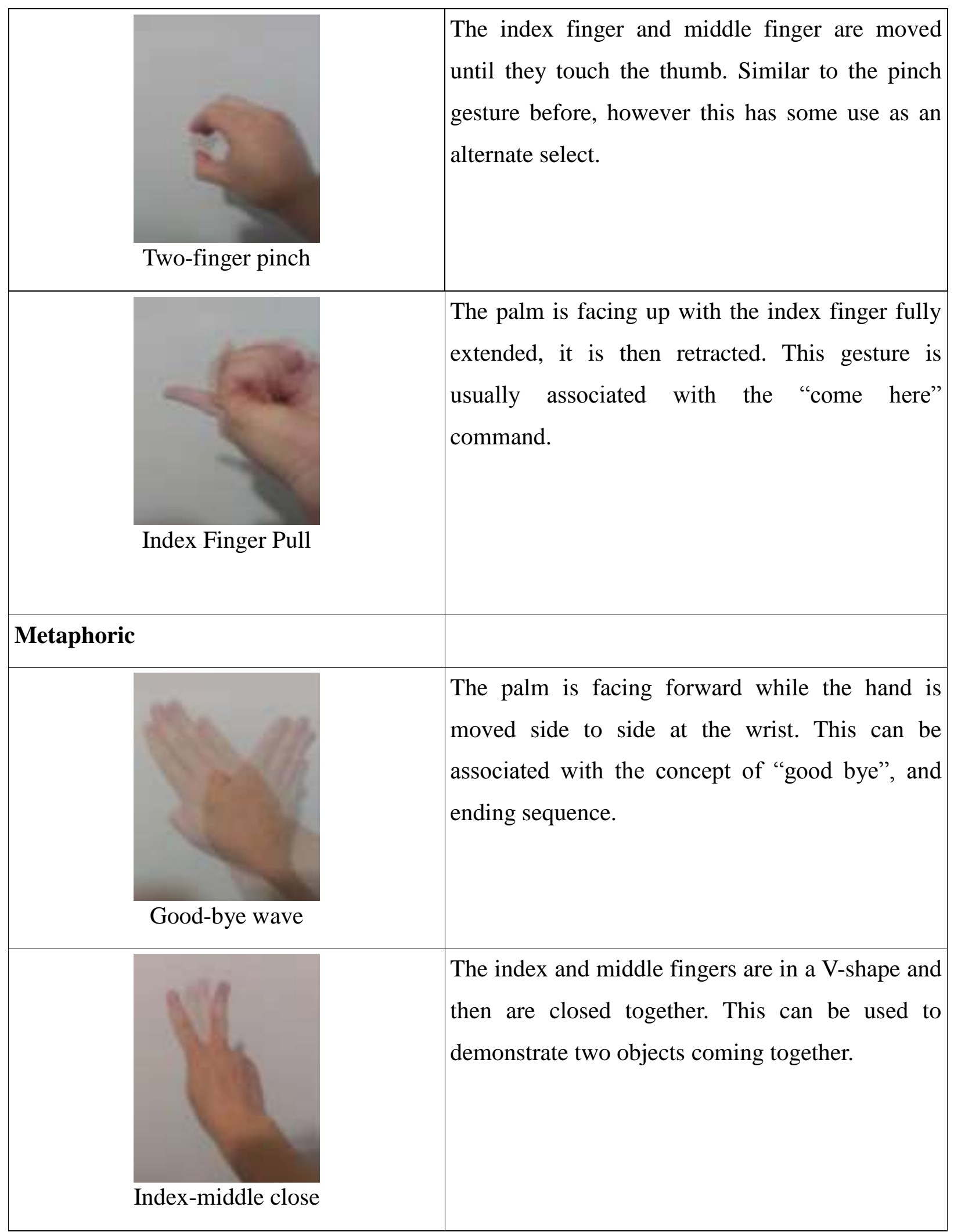




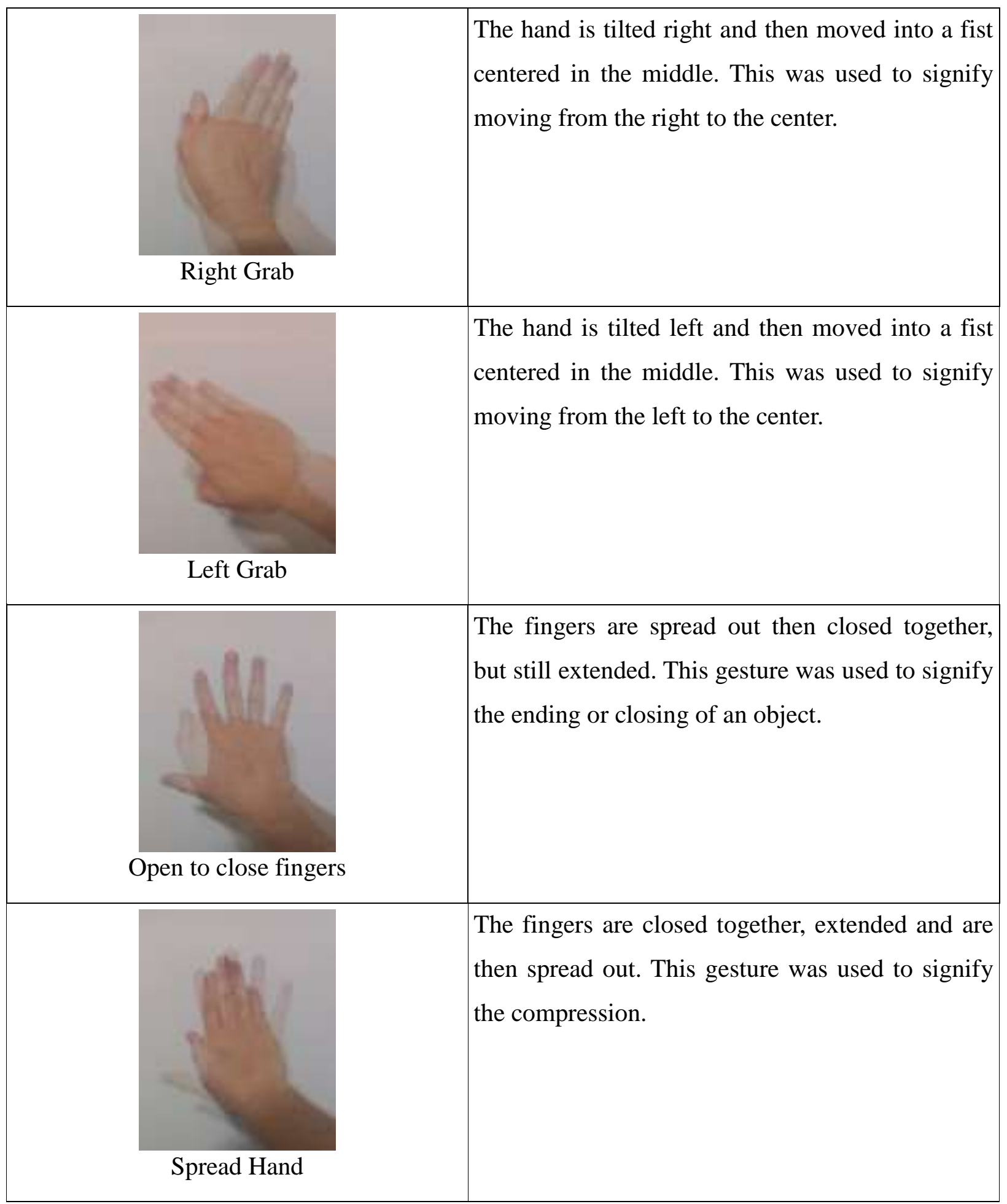




\begin{tabular}{|l|l|}
\hline Arm side to side & $\begin{array}{l}\text { The wrist and hand are in fixed position while the } \\
\text { arm moves them side to side. This gesture used to } \\
\text { signify the shaking or movement of an object. }\end{array}$ \\
\hline Wrist Tilt & $\begin{array}{l}\text { The hand is in neutral position and is tilted left } \\
\text { and right at the wrist. This object was used to } \\
\text { demonstrate the rotation or axial movement of an } \\
\text { object. }\end{array}$ \\
\hline Feictic & $\begin{array}{l}\text { The hand tilted in the desired direction. This } \\
\text { gesture is used as a command to signify when to } \\
\text { move to the desired direction. The object or } \\
\text { concept is instructed to stop, when the hand tilts to } \\
\text { a direction, it is instructional for the object to } \\
\text { move in that direction. }\end{array}$ \\
\hline then pointed at a desired direction. \\
\hline
\end{tabular}




\begin{tabular}{|c|l|}
\hline Palm & $\begin{array}{l}\text { With the fingers extended, the palm is pushed into } \\
\text { the desired direction. Used as a command to push } \\
\text { an object in the desired direction. }\end{array}$ \\
\hline Moving Point & $\begin{array}{l}\text { With the index finger extended, the entire hand is } \\
\text { moved forward in the direction that the finger is } \\
\text { pointing in. }\end{array}$ \\
\hline
\end{tabular}

Table 4.1: Categorization of compiled database gestures

In Table 4.1 ASL gestures were grouped into the single grouping as the gestures carried similar meaning but for different symbols. The full description of all ASL gestures used in this study can be referred to in Appendix 1, Table 4.2. For the compilation of this database, gestures of similar shape and style were omitted when found in later databases. As a study into the exertion of gestures, different hand positions and styles were considered to be more preferable to minor variants of similar gesture groups. The compilation of the databases gestures demonstrate that simulated object manipulation is the preferred of interaction for database systems. As seen in previous research, the manipulation of virtual objects via gesture recognition offers context and background for the system to be more effectively utilized. For systems that operate on static gestures map them to predefined commands. Static gestures are usually universal in their meanings, such as stop, positive/negative response, and directional. As such there were no metaphoric gestures found in the databases that utilized static gestures. Dynamic gestures were found to have more variations and more complex commands are assigned to them. Given their nature, it was found that context is very important for these gestures as the information given by 
one dynamic gesture can offer multiple interpretations. Some gestures are universal in their roles such as simulated object manipulations, or conventional knowledge. Dynamic gestures also offer several variations that had no specific meaning nor could be interpreted without context provided. These gestures were not commonly found in databases they were mapped to commands that were not reflective of the shape or action of the gesture.

\subsection{Gesture Exertion on User}

In chapter 3, the use of the Myo Armband was introduced in measuring exertion by the subject. To accomplish this, the subject first established two reference points, a relaxed rest posture and then made full exertion in a tight fist. Both values were recorded and assigned values based on readings measured. Initial values were ignored and only the steady state was measure and recorded. Due to involuntary muscle twitch and other factors, the exclusive inclusion of the steady state was ideal. Figure 4.1 demonstrates the graph obtained from full exertion by the subject.
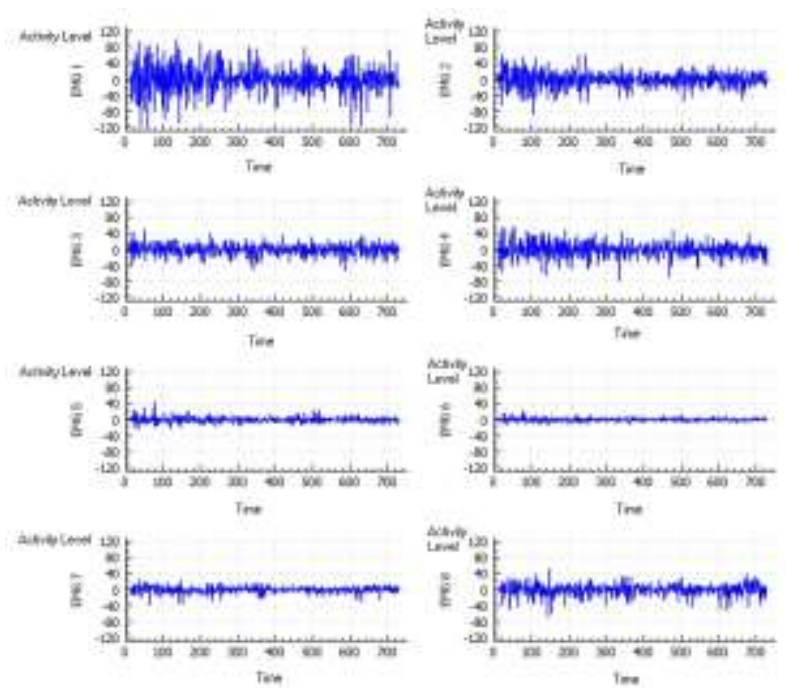

Figure 4.1: Myo Armband EMG reading for full exertion

The recording would begin when the subject has made a gesture and held the posture for a one to two seconds, after holding the posture until a steady state has been recorded, the subject 
will then adopt the rest posture and wait for the readings to reach their rest states, then the subject will perform another gesture and hold the posture until a steady state has been reached. This process is repeated for all static gestures and their values recorded, Table 4.2 is a graphical representation of the data obtained during trials for ASL B and ASL C.
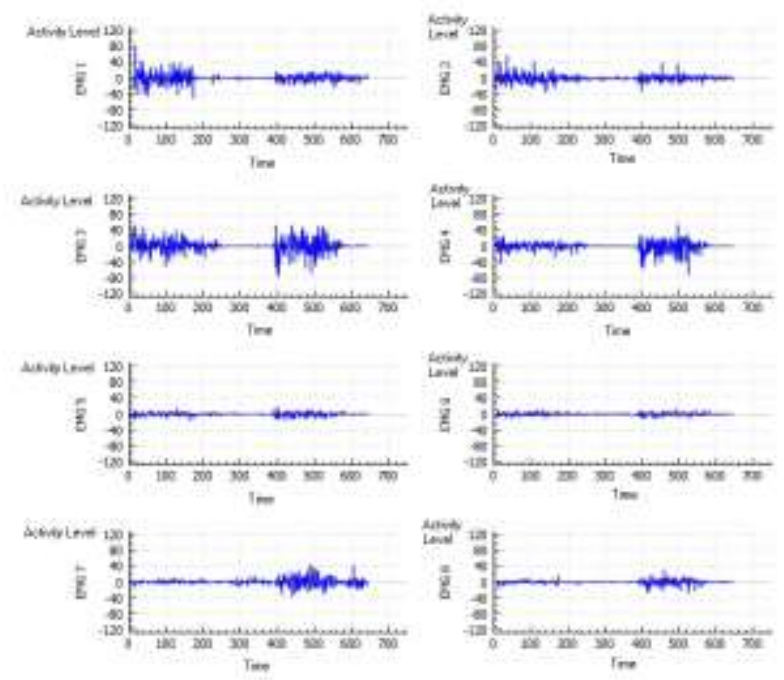

Figure 4.2: Myo Armband EMG readings for:

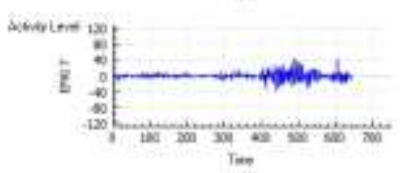

ASL B and ASL C

For dynamic gestures, due to their nature do not have a steady state, however the gestures were performed repeatedly for several samples, the average peak of these values were then recorded. As with static gestures, the subject then adopted a rest position for several seconds before making the next gesture. This was repeated until all dynamic gestures were recorded, Figure 4.3 demonstrates the data obtained during dynamic gestures. 

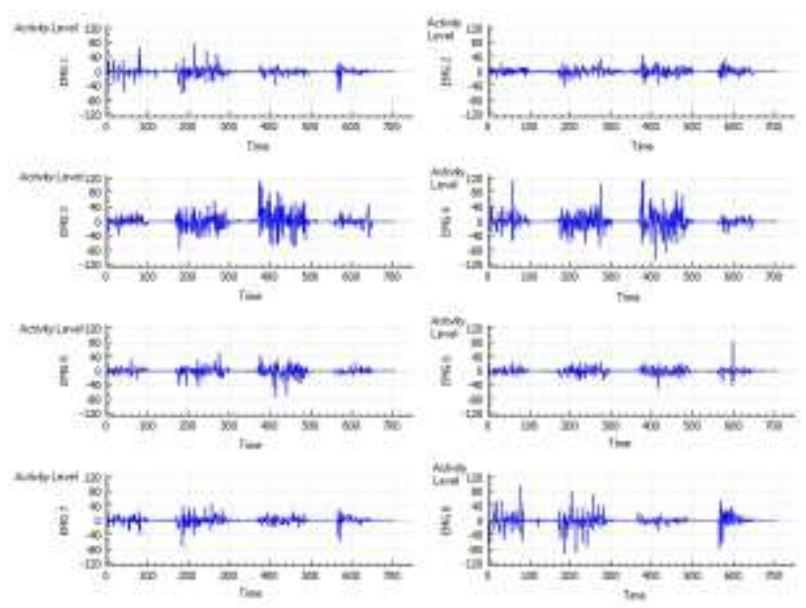

Figure 4.3: Myo Armband EMG readings for:

Tilt, Fork Tilt, Palm and Moving Point

In Appendix 2, Table 4.5, graphical data regarding the Activity Levels of all gestures are recorded. They are similar to the data obtained by the averaging software, however not all gestures recorded in the table will match with the data in Table 4.2 and Table 4.4 due to the inability of the human body to perfectly replicate a specific action. The data obtained from the Myo Device used in Table 4.2 and Table 4.4 were averages of several iterations of gesture making while the graphs in Table 4.5 are single snap shots of a single iteration during the trials. Data should be obtained by the average of several iterations as it serves as a better comparison range. A plot of the finger position difference with respect to exertion was constructed with Equation 4.1 where AFD is the Average Finger position Difference, I is the index finger position, $\mathrm{M}$ is the middle finger position, $\mathrm{R}$ is the ring finger position, $\mathrm{L}$ is the little finger position, $\mathrm{TF}$ is the thumb flex, TA is the thumb axis and $\mathrm{W}$ is the wrist movement. This equation takes into consideration the distance between adjacent fingers, since the thumb and wrist are independent agents the position they are away from the neutral position can attribute to exertion, they are separately considered in the equation. The difference will be plotted with respect to exertion.

$$
A F D=|(I-M)|+|(M-R)|+|(R-L)|+|T F|+|T A|+|W|
$$




\subsubsection{High Exertion}

In the study conducted on exertion, a tight fist was made with the effort of maximum exertion by the subject; the value obtained in this trial was a total of 460 as demonstrated by the readings while the rest position given its value is declared to be 0 . However due to the nature of the exertion, a stable reading at the highest possible exertion level could not be read, instead the highest exertion for steady state was obtained, that is the subject was able to sustain the gesture for an extended period of time. It should be noted that above the total value of 300 , the subject felt uncomfortable and thus this was used to define a high exertion gesture. Table 4.2 contains all gestures from the trial that were identified as high exertion.

\begin{tabular}{|c|l|l|l|l|l|l|l|l|l|}
\hline Gesture & Pod 1 & Pod 2 & Pod 3 & Pod 4 & Pod 5 & Pod 6 & Pod 7 & Pod 8 & Total \\
\hline ASL B & 20 & 40 & 80 & 70 & 30 & 20 & 40 & 30 & 330 \\
\hline ASL K & 30 & 90 & 90 & 90 & 70 & 30 & 30 & 40 & 470 \\
\hline ASL M & 50 & 80 & 80 & 60 & 20 & 20 & 10 & 30 & 350 \\
\hline ASL W & 50 & 50 & 70 & 80 & 40 & 30 & 30 & 40 & 390 \\
\hline Stop Gesture & 40 & 50 & 100 & 80 & 40 & 30 & 30 & 20 & 390 \\
\hline Wave Right & 40 & 70 & 100 & 100 & 50 & 40 & 30 & 30 & 460 \\
\hline Wave Left & 60 & 20 & 40 & 20 & 20 & 20 & 80 & 40 & 300 \\
\hline Grab & 90 & 80 & 80 & 80 & 30 & 20 & 30 & 50 & 460 \\
\hline Knocking & 60 & 60 & 50 & 50 & 20 & 20 & 40 & 40 & 340 \\
\hline Arm side to side & 60 & 60 & 70 & 40 & 20 & 20 & 10 & 20 & 300 \\
\hline
\end{tabular}

Table 4.2: High Exertion Gestures (Activity Levels)

High exertion gestures demonstrate three key configurations, the first of which is the fingers fully extended with large movements of the wrist, the second is a fist gesture that uses large movement of the wrist, and the third are adjacent fingers in opposite direction to one another. Other gestures that do not demonstrate these features, the exertion values are lower and thus considered more comfortable. Figure 4.4 shows dynamic gestures the exertion values vs the finger difference. Simulated object manipulation can be considered a special case, and observing non-object oriented gestures show that generally, as exertion values increase so do average finger difference. It should be noted that some of these gestures are not able to be made quickly 
and in some cases require assistance from the other hand to aid in forming the proper shape. As shown in EMG graphs taken during the experiment these gestures also require a significant effort to maintain and sustained used of can possibly injure the user. Gestures that invoke this level should be avoided or used sparingly.

\begin{tabular}{|c|l|l|l|l|l|l|l|}
\hline Gesture & Thumb Axis & Thumb Flex & Index & Middle & Ring & Little & Wrist \\
\hline ASL B & -10 & 10 & 10 & 10 & 10 & 10 & 0 \\
\hline ASL K & -5 & 8 & 10 & 10 & -10 & -7 & 0 \\
\hline ASL M & -10 & 0 & -6 & -6 & -6 & -10 & 0 \\
\hline ASL W & -10 & -10 & 10 & 10 & 10 & -10 & 0 \\
\hline Stop Gesture & -4 & -7 & 10 & 10 & 10 & 10 & 0 \\
\hline Wave Right & 0 & 0 & 10 & 10 & 10 & 10 & 10 \\
\hline Wave Left & 0 & 0 & 10 & 10 & 10 & 10 & 10 \\
\hline Grab & -10 & -10 & -10 & -10 & -10 & -10 & 0 \\
\hline Knocking & -10 & -10 & -10 & -10 & -10 & -10 & 10 \\
\hline Arm side to side & -10 & -10 & -10 & -10 & -10 & -10 & -10 \\
\hline
\end{tabular}

Table 4.3: High Exertion Gestures Movement Range

One observation made is that high exertion static gestures were considered to exertion the user more. As seen in the EMG graphs obtained during the experiment, the peak exertions for dynamic gestures occur in a smaller time frame and because of the variable nature of dynamic gestures there are areas where the gesture is actually in a rest posture. Another factor noticed in the experiment is the use of wrist movements may cause higher values as most muscles are used during the movement. However, these gestures are still shown to be uncomfortable as key features of these high exertion gestures are the stressful positions taken by the hand and sudden large movements in relation to the hand and wrist. 


\subsubsection{Low Exertion}

\begin{tabular}{|c|l|l|l|l|l|l|l|l|l|}
\hline Gesture & Pod 1 & Pod 2 & Pod 3 & Pod 4 & Pod 5 & Pod 6 & Pod 7 & Pod 8 & Total \\
\hline ASL G & 20 & 10 & 10 & 20 & 10 & 10 & 10 & 10 & 100 \\
\hline ASL H & 10 & 20 & 40 & 20 & 10 & 10 & 40 & 10 & 160 \\
\hline ASL N & 20 & 20 & 40 & 20 & 10 & 10 & 10 & 10 & 140 \\
\hline ASL O & 10 & 20 & 40 & 30 & 10 & 10 & 10 & 10 & 140 \\
\hline ASL P & 20 & 10 & 10 & 10 & 10 & 10 & 40 & 20 & 130 \\
\hline ASL S & 50 & 20 & 50 & 20 & 10 & 10 & 10 & 10 & 180 \\
\hline ASL X & 30 & 20 & 20 & 40 & 10 & 10 & 10 & 20 & 160 \\
\hline Fingers Closed & 20 & 10 & 30 & 30 & 10 & 10 & 30 & 30 & 170 \\
\hline Thumbs up & 20 & 20 & 30 & 30 & 20 & 10 & 30 & 30 & 190 \\
\hline Thumbs Down & 20 & 10 & 30 & 30 & 10 & 10 & 20 & 20 & 150 \\
\hline Point & 20 & 20 & 30 & 20 & 10 & 10 & 20 & 30 & 160 \\
\hline Hand point & 20 & 20 & 40 & 30 & 10 & 10 & 20 & 20 & 170 \\
\hline Two-Finger Point & 20 & 20 & 40 & 40 & 10 & 10 & 20 & 20 & 180 \\
\hline Lever Control & 20 & 30 & 30 & 30 & 20 & 20 & 20 & 10 & 180 \\
\hline Wave Up & 10 & 10 & 30 & 30 & 10 & 10 & 40 & 20 & 160 \\
\hline Lever Spin & 20 & 40 & 40 & 30 & 10 & 10 & 10 & 10 & 170 \\
\hline Lever Pull & 10 & 20 & 30 & 30 & 10 & 10 & 10 & 10 & 130 \\
\hline Switch & 40 & 30 & 30 & 20 & 10 & 10 & 20 & 20 & 180 \\
\hline Pinch & 20 & 30 & 30 & 40 & 20 & 10 & 10 & 20 & 180 \\
\hline Spread Pinch & 20 & 20 & 20 & 20 & 10 & 10 & 10 & 20 & 130 \\
\hline Two-finger pinch & 20 & 30 & 40 & 30 & 20 & 20 & 10 & 10 & 180 \\
\hline Index Finger Pull & 20 & 10 & 10 & 20 & 20 & 10 & 30 & 10 & 130 \\
\hline ASL J & 10 & 10 & 20 & 20 & 10 & 10 & 20 & 20 & 120 \\
\hline ASL Z & 20 & 40 & 30 & 20 & 10 & 10 & 20 & 20 & 170 \\
\hline Moving Point & 20 & 20 & 20 & 20 & 10 & 10 & 20 & 30 & 150 \\
\hline
\end{tabular}

Table 4.4: Low Exertion Gestures (Activity Levels)

In the study the rest position demonstrated a value close to 0 , and for the purposes of this study had been declared to be 0 for simplicity, however, in readings that demonstrated values of 10 to 20 , the subject declared that the exertion and effort felt similar to the rest position, that is very relaxed and did not require exertion from the user. It is also noted that readings of 30 demonstrated both rest and exertion features, though it was not enough to be considered uncomfortable. Due to this, total EMG values below 200 were considered to be low exertion and 
would be ideal for prolonged use in an interactive system. As previously noted, the relative positioning of the fingers can reflect exertion individually, hence why a value of 200 was selected. This accounts for gestures that relax certain aspects while exerting others.

Several features noted in low exertion gestures show several key configurations, the first and most noticeable are gestures that closely resemble the rest position. In the case of the lever gestures, the user's hand is close to the rest gesture's shape, the fingers are in the middle movement range the thumb is neither stretched or flexed, the simulation of an object in the grasp of the user allows the hand to more closely resemble its rest position, allowing gestures with this feature to exert the user significantly less. The second feature is that the fingers should be close to each other in terms of flex; that the more fingers are in the same direction an increase in comfort can be expected. As such gestures that only require one finger to be extended are generally required less exertion from the user. The third feature is the use of the entire arm to make motions, for gestures that have stationary shapes but feature motion from the arm show little exertion on the user, however, an argument can be made that exertion will instead be placed on the shoulder, it is also a considerably larger muscle mass and is more resistant to exertion, though this is a possible future topic of study. Figure 4.4 also demonstrates that simulated object manipulations gestures, or object oriented gestures score consistently lower exertion values. It also does not follow the general trend of higher finger differences leading to higher exertion values. Further investigation and study into this observation can be considered for future work. 


\begin{tabular}{|c|r|r|r|r|r|r|r|r|}
\hline Gesture & Thumb Axis & Thumb Flex & \multicolumn{1}{l}{ Index } & \multicolumn{1}{l}{ Middle } & Ring & Little & Wrist \\
\hline ASL G & 0 & 0 & 0 & -10 & -10 & -10 & 3 \\
\hline ASL H & -10 & 10 & 10 & 10 & -10 & -10 & 4 \\
\hline ASL N & -10 & 0 & -6 & -6 & -10 & -10 & 0 \\
\hline ASL O & 0 & 0 & -1 & -1 & -1 & -1 & 0 \\
\hline ASL P & 10 & 4 & 10 & 10 & -10 & -10 & 8 \\
\hline ASL S & -10 & -10 & -10 & -10 & -10 & -10 & 0 \\
\hline ASL X & 0 & -7 & 5 & -10 & -10 & -10 & 0 \\
\hline Fingers Closed & 0 & -7 & 10 & 10 & 10 & 10 & 0 \\
\hline Thumbs up & 10 & 10 & -10 & -10 & -10 & -10 & 0 \\
\hline Thumbs Down & 10 & 10 & -10 & -10 & -10 & -10 & 0 \\
\hline Point & -10 & -10 & 10 & -10 & -10 & -10 & 0 \\
\hline Hand point & 10 & 10 & 10 & 10 & 10 & 10 & 0 \\
\hline Two-Finger Point & -10 & -10 & 10 & 10 & -10 & -10 & 0 \\
\hline Lever Control & 1 & 1 & 1 & 1 & 1 & 1 & 0 \\
\hline Wave Up & 0 & 0 & 10 & 10 & 10 & 10 & 10 \\
\hline Lever Spin & 1 & 1 & 1 & 1 & 1 & 1 & 10 \\
\hline Lever Pull & 1 & 1 & 1 & 1 & 1 & 1 & 0 \\
\hline Switch & -10 & -10 & 10 & -10 & -10 & -10 & 0 \\
\hline Pinch & 3 & 0 & -3 & -10 & -10 & -10 & 0 \\
\hline Spread Pinch & 3 & 0 & -3 & -10 & -10 & -10 & 0 \\
\hline Two-finger pinch & -5 & -5 & -5 & -5 & -10 & -10 & 0 \\
\hline Index Finger Pull & -10 & -10 & -10 & -10 & -10 & -10 & 0 \\
\hline ASL J & 10 & 10 & -10 & -10 & -8 & 10 & 8 \\
\hline ASL Z & -5 & -10 & 7 & -10 & -10 & -10 & 0 \\
\hline Moving Point & -10 & -10 & 10 & -10 & -10 & -10 & 10 \\
\hline
\end{tabular}

Table 4.5: Low Exertion Gesture Movement Range

Some low exertion gestures share physical features with high exertion gestures, one such feature is the majority or all fingers in a fist shape, and the reason for this is the hand does not require additional movements that may cause the hand to exert more effort to maintain the shape and form. In dynamic gestures the action and movement of the hand is slower even when it reaches the range limit of their respective features. 
Finger Difference vs Exertion (Dynamic)

O bject vs Non-O bject

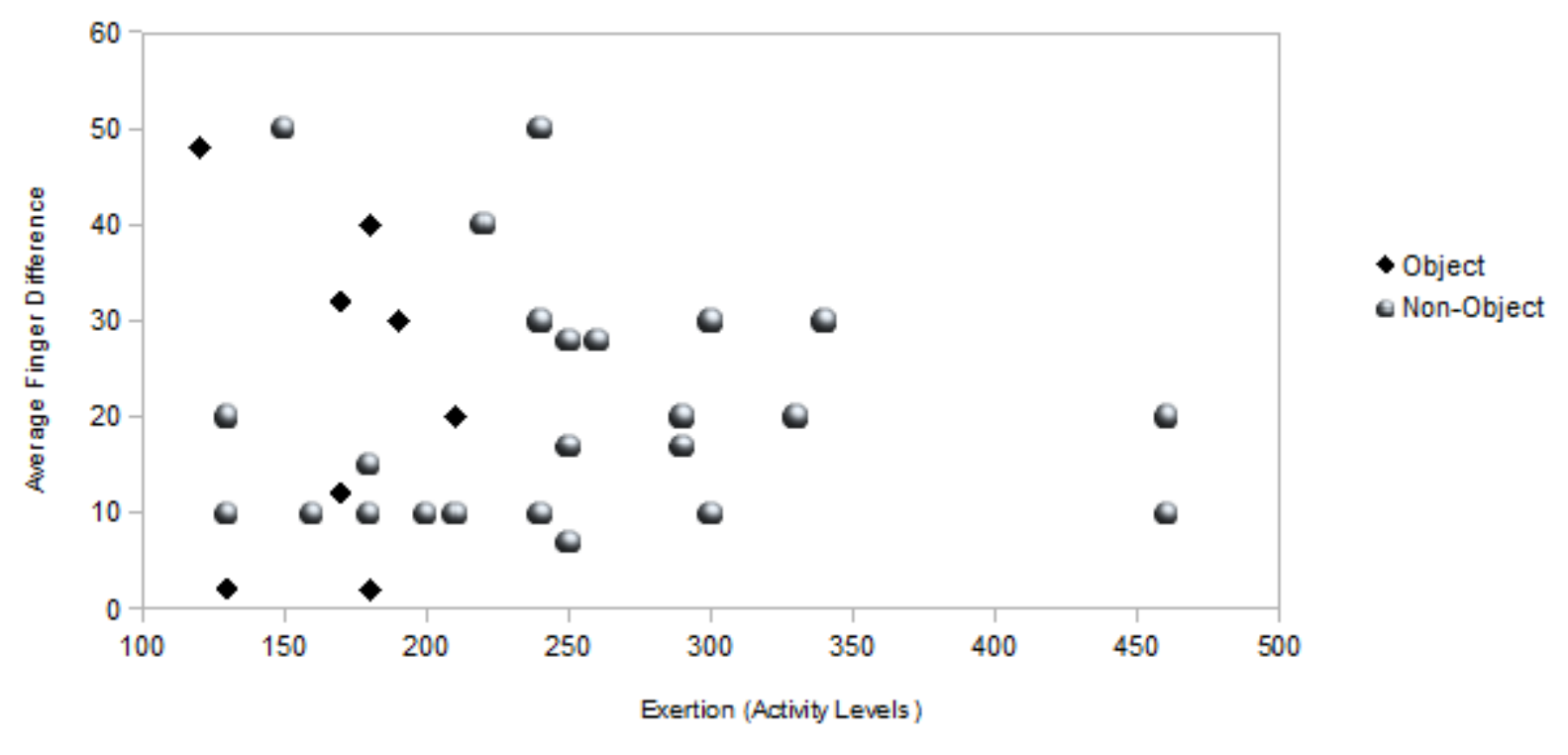

Figure 4.4: Finger Difference vs Exertion (Dynamic)

\subsection{Summary}

In the study of current gesture database designs, several factors were considered, the gesture type, classified into static and dynamic gestures and then further categorized into iconic, metaphoric and deictic in order to identify and provide a frame work comparisons in later studies, Table 4.1. The exertion and movement range was then investigated through the use of a Myo armband sensory device to investigate the ergonomic design of the gestures. Through the use of these investigations, a guideline of how gestures should be made was formed.

The gestures were classed into iconic, metaphoric and deictic, and then further sub divided into static and dynamic. These classifications help understand the general meaning and specific application each gesture has. It was found that static gestures selected in these categories were either universal in nature or operate on conventional knowledge. Dynamic gestures relied heavily on context to convey information and due to their complex nature, 
allowed for more abstract actions and shapes to be performed in relation to a command. Table 4.1 and Table 4.2 in Appendix 1 comprised of 66 gestures obtained from various databases, where Table 4.2 referred to the collection of ASL letters. From this categorization it can be noted that current development into gesture recognition is heading towards dynamic gestures that operate in a virtual 3 dimensional environment where the user of the system will interact with objects and tools to perform certain tasks or issue commands [56][58]. As seen in the study into exertion and finger positions, gestures that aim to interact with virtual or simple real world objects achieve lower exertion values than gestures that aim to replicate object shapes.

Following the gesture classification, the exertion and movement range of each gesture was investigated through the use of an EMG sensory device, the Myo Armband. The armband provided the EMG readings that correlated with the exertion the user experienced during the gesture making and maintaining process. Using this information, the relative exertion of the gesture could be investigated. In conjunction with the exertion investigation, the movement range of the gestures in reference to the fingers and wrist was also investigated. Using rest, extended and compressed postures as reference points, each gesture was examined in relation to the limits of the subject's physical characteristics. Using this information, high exertion gestures were identified and compared to their movement range analysis. It was discovered that high exertion gestures contained specific features. For static gestures, these were fingers being in vastly different orientations from each other, while in dynamic gestures these were gestures that heavily used the wrist in addition to reaching the upper physical limits of the hand. Following the investigation of high exertion gestures, investigation of low exertion gestures was carried out. For static gestures, the fingers tend to be in the same orientation as their adjacent fingers, as well as being close to the rest position in shape. For dynamic gestures, the inclusion of a simulated tool in the user hand helped to maintain the shape of the rest posture or a relaxed shape for the hand; this enabled these gestures to have rest periods in between active regions. Furthermore, simulated object manipulation gestures demonstrate much lower exertion values; this can be a consideration when designing gestures for computer interactions. 


\section{Chapter 5}

\section{Discussions and Conclusions}

This thesis implemented a method of examining exertion with respect to gesture design with a goal of improving gesture interaction interfaces. The development of gesture classification aids in designing gesture on an application level while the exertion and gesture design found and identified flaws in current gesture design work. Through this study, improvements to current gesture designs have been created.

\subsection{Design Guidelines}

From the study above observing high exertion and low exertion gestures, the following guidelines should be considered when designing gestures for recognition systems.

1. Intuitive: The gesture should be fairly universal in meaning if it does not invoke imagery, conventional gestures can be used, common gesture interactions with other technology can be considered if the use is widespread and considered easily recognizable. Such examples would be touch gestures used in touch-screen surfaces, as the technology is widespread and the cultural influence invoked by it has reached a larger audience.

2. Rest Posture: Dynamic gestures should consider movement ranges that fall in the rest area, this is the area of least exertion for the user and by placing most of the actions in 
this region, and the exertion of the gesture on the user can be greatly reduced. Additionally, continuous gesture making should invoke rest posture as a means of starting and ending a gesture, this enables the system to better recognize the actions of the user and offers better control for the user to give meaningful commands to a recognition system.

3. Ease of Maintenance: For gestures that require a longer period of time to stay in one shape, the gesture should consider the following features: fingers should be mostly aligned in the same or similar orientation with their adjacent fingers, movements should only contain the necessary movement for a large enough physical change for the system to recognize, and only gestures that are close to rest posture should be held for an extended period of time.

4. Simulated object manipulation: When given context, gestures can provide more information than by verbal or other means; the use of virtual simulated object manipulation by gestures is a powerful tool that enables comfort, intuitiveness, and understanding. As demonstrated, gesture commands have a large development in gestures that simulate the operation of objects or tools in the virtual space. As observed in Figure 4.4 in Chapter 4, Simulated object manipulation Gestures show lower exertion values than most non-object oriented gestures. Due to simulated object manipulation gestures relating to concrete concepts, they are all classified as iconic gestures.

One criticism for visual gesture recognition is the uncomfortable or unfamiliar employment of if in these systems; the attempt to replace more traditional input methods that are more efficient is a major factor in these criticisms. Designers and developers need to pay special attention to the designs and method of implementation, if the system serves no purpose beyond incorporating the technology into the product, then it will detract from the quality and experience. This study was made in the hope that these developers create systems that enhance the experience.

As technology continues to develop, it is also important to create systems that are safe and comfortable for the users. The promotion of ergonomic gestures will help to improve upon the technology as well as create new conventions for later systems. As the technology sees more 
widespread use, it is important to carefully design these gestures for these systems, both for the growth of the technology and future work that will be built upon it.

\subsection{Possible Future Work}

This study investigates and presents gesture designs guidelines as a study of their context and effects on the user. Future work related to this study will see an expanded study into different or specific gesture types or databases. Furthermore, gesture recognition technology will further expand into other languages that the body can produce. Using this study as a basis, intelligent designs of gesture and body language for computer recognition can be carried out. Some of the following studies can be performed to further refine these guidelines.

Investigation into specific target audiences, such as hearing impaired or mute persons will provide extensive materials into creating systems that can interpret language more effectively. As seen in this study, sign language gestures are not always comfortable for the user and several of these gestures are high exertion, thus extended use of these for any system will be uncomfortable and cause undo exertion on the user. Studies such as this can be applied to the gestures that will eventually be developed into systems for use for specific target audiences and users. Currently little study has been made into object oriented gestures, as observed in this thesis, simulated object manipulation demonstrated much lower exertion values than non-object oriented gestures. Study into this can yield valuable information into comfortable gesture designs for dynamic gestures.

A study into databases with specific applications will further refine the guidelines for different applications. As discussed previously, context to gesture recognition is a valuable part of the information delivered by the system. Specific applications can include entertainment purposes, design software, navigation software, and control systems. At the basis of all of these applications, it can be inferred that the user may spend an extended period of time operating the system, hence the ergonomic design catered for these applications should be considered. By conducting studies such as these using subjects of expert in their fields, gesture recognition will see a much wider spread of use given that camera technology is also widespread and seeing an 
increase in use.

A later study can be conducted is which users can be given a list of commands and then asked to perform a gesture related to the command. This can add a layer of intuitiveness if a large sample of subjects were to be used in this study. By observing the user expectations, it is possible to compare and contrast with developer expectations to enhance the development and design of gestures in these systems. This is a possible extension of this thesis as this observes and analysis gestures in the developer domain. A study into user derived gestures can demonstrate more effective gestures.

\subsection{Conclusion}

For the purposes of investigating gesture designs in computing systems for human-computer interactions, several current instructional gesture databases was obtained and investigated. With the goal of classifying and understanding gesture use in computer interactions, several aspects of gestures from these databases were investigated. This thesis then draws conclusions from the results obtained from this investigation.

For this research, different aspects of gesture recognition were investigated. Current technology and trends were observed and the best approach to gesture recognition was considered based on this research. Notably, this included an examination of recognition techniques; required hardware and related body language interpreters such as gaze and touch gestures. With the conclusion that camera based systems will reach the largest number of audiences given its wide distribution, gesture types were investigated and examined. Through this examination, and understanding of the relation between computer interfaces and gestures can be observed. In order to effectively accomplish this, some research was made in how gestures and languages are related, terms, ideas and metrics from this section will be used to examine and observe gestures. Finally, the design of gestures was investigated, from here, different aspects of gestures were examined to help reconcile the terms required to understand how body language and gestures convey information to computing systems.

In this study of gestures in current recognition systems, several public databases were 
obtained and analyzed. In this analysis, the database gestures were extracted and categorized. Gestures that were similar in shape and function to ones already categorized were discarded. From this analysis, the gestures were separated into categorizations for further analysis. It was discovered that static gestures tend to use universal signs and shapes while dynamic gestures offer a greater range of information. With the gestures organized into categories, the exertion and movement range was analyzed using EMG sensor equipment and photo analysis. Through this analysis, exertion and finger locations were compared and contrasted. It was found that high exertion gestures involved sudden motions from one physical limit to another as well as opposite positioning to adjacent features. For low exertion gestures, the motions involved were more relaxed as well as finger positions in orientations close to the rest position. Furthermore, low exertion gestures have long periods of rest with only occasional exertion functions, however, it should also be noted that these exertion functions are still relatively low compared to readings seen in high exertion gestures. Findings and results of this study are similar to those found in [21] when comparing the analysis of the ASL gestures. With interest in gesture recognition increasing, development into recognition techniques is ever rising. All aspects of gesture recognition are being explored to determine its scope and potential, however much emphasis has been placed on the technical facets leaving the interaction aspect unexplored. However advance the technology it is still ultimately up to the end user of the system that determines much of that potential. Without considering the needs and comfort of the user, the technology's potential will be held back. As with all technology, it is the users that control its future. 


\section{Appendix 1}

\section{Compiled Gesture Database}

Gestures taken from multiple databases were compiled into this list, an image of the gesture and its description was provided to keep consistency during testing.

\begin{tabular}{|l|l|}
\hline Gesture & Description \\
\hline Iconic & $\begin{array}{l}\text { American Sign Language, each symbol used in } \\
\text { this set is a representation of a letter in the English } \\
\text { alphabet. These are usually used in place of a } \\
\text { keyboard to type or enter a character into an } \\
\text { interactive system. }\end{array}$ \\
\hline ASL & $\begin{array}{l}\text { A forward facing palm that is normally associated } \\
\text { with the command or request to stop. }\end{array}$ \\
\hline Stop Gesture & \\
\hline
\end{tabular}




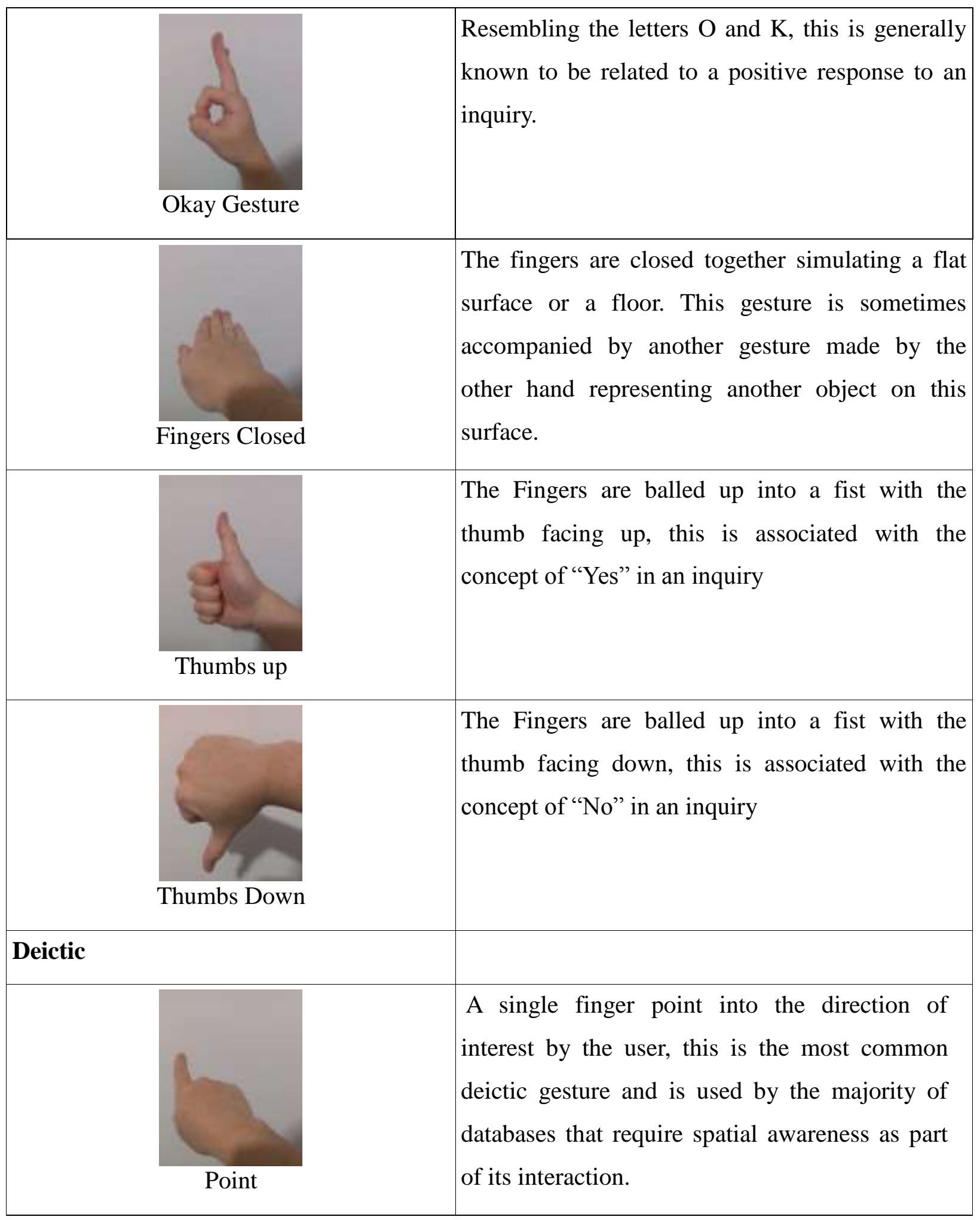




\begin{tabular}{|l|l|}
\hline Hand point & $\begin{array}{l}\text { Using all fingers and thumb to point to specific } \\
\text { direction, this is a highly uncommon pointing } \\
\text { gestures but it is sometimes used when confirming } \\
\text { four or more objects are related to the direction } \\
\text { identified. }\end{array}$ \\
\hline Dynamic: & $\begin{array}{l}\text { Using two fingers to point in specific direction, } \\
\text { this deictic gesture is normally interchangeable } \\
\text { with the single point gesture; however, sometimes } \\
\text { it is used to declare two objects in relation to the } \\
\text { identified direction. }\end{array}$ \\
\hline Iconic & $\begin{array}{l}\text { The hand is in a neutral position and makes a } \\
\text { circular motion right at the wrist. This simulates } \\
\text { an object rotating right. }\end{array}$ \\
\hline Circular Right & $\begin{array}{l}\text { The hand is in a neutral position and makes a } \\
\text { circular motion left at the wrist. This simulates an } \\
\text { object rotating left. }\end{array}$ \\
\hline
\end{tabular}




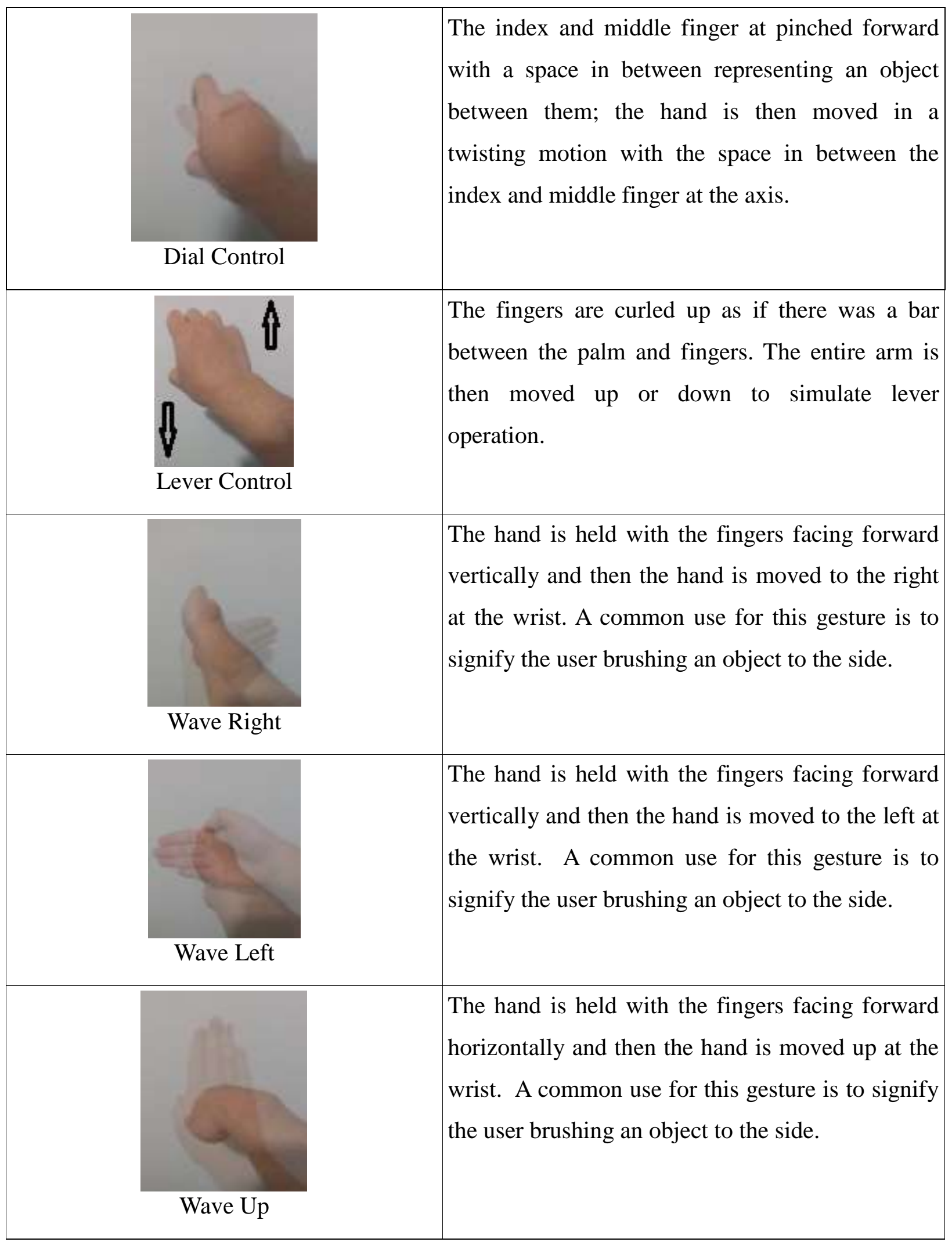




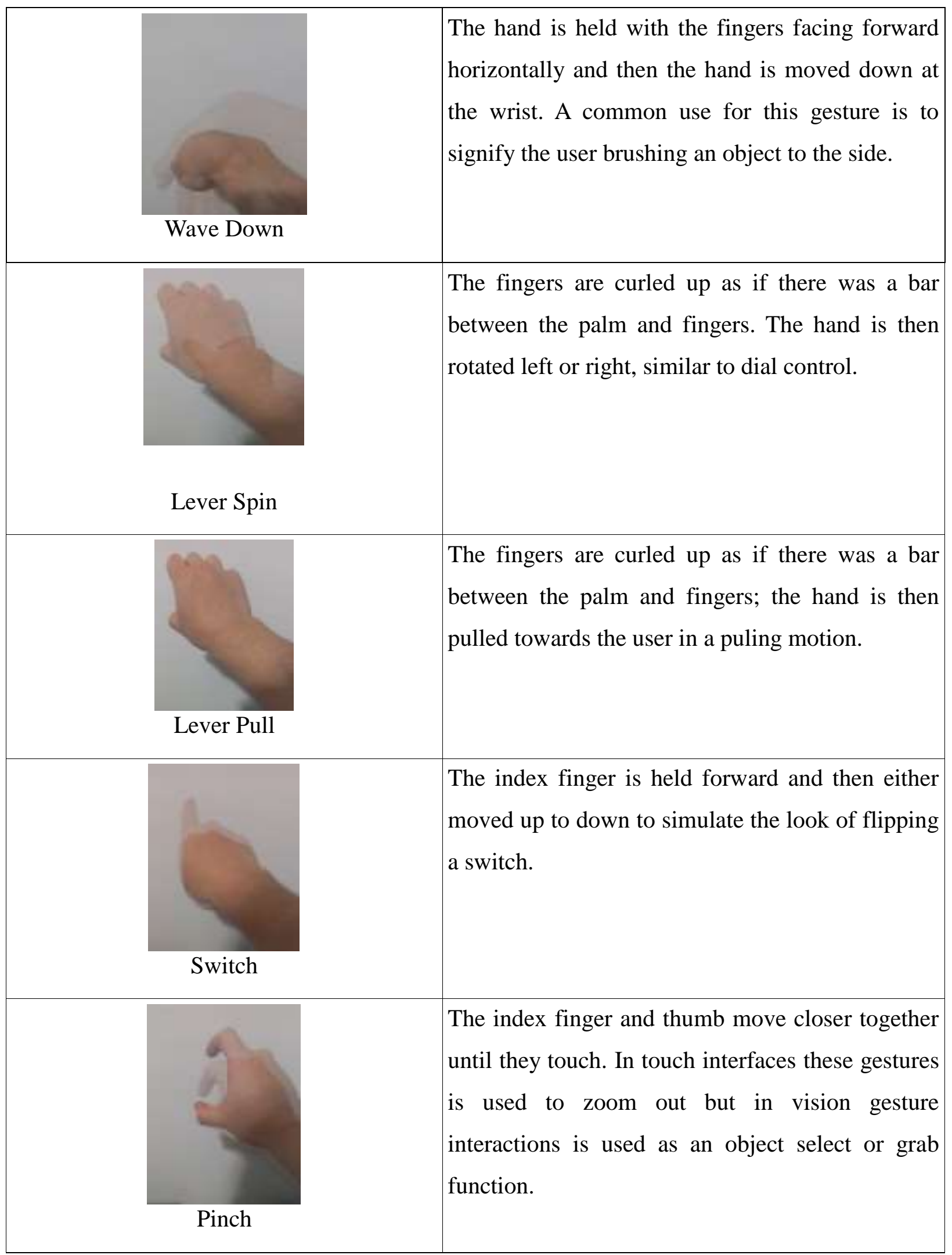




\begin{tabular}{|l|l|}
\hline Spread Pinch & $\begin{array}{l}\text { The index finger and thumb move further apart } \\
\text { from a touching position. In touch interfaces this } \\
\text { gesture is often used with the zoom in feature, } \\
\text { however, in vision gesture interaction systems; } \\
\text { this is used to release an object under select. }\end{array}$ \\
\hline Grab & $\begin{array}{l}\text { The fingers are open and then are closed into a } \\
\text { fist. This gesture is commonly use with the select } \\
\text { command and used to select an object in virtual } \\
3 \text { space. }\end{array}$ \\
\hline Press down & $\begin{array}{l}\text { The palm is faced down and the entire hand is } \\
\text { moved down. Used for pushing an object down or } \\
\text { to signify the lowering of an object. }\end{array}$ \\
\hline Lift Up & $\begin{array}{l}\text { The entire arm is rotated back and forth. This } \\
\text { gesture is commonly associated with object } \\
\text { rotation. } \\
\text { raising of an object. }\end{array}$ \\
\hline The palm is faced up and the entire hand is moved \\
\hline
\end{tabular}




\begin{tabular}{|l|l|}
\hline Knocking & $\begin{array}{l}\text { A fist is made with the palm facing forward; the } \\
\text { fist is then moved back and forth at the wrist. This } \\
\text { gesture is used to simulate the knocking of a door. }\end{array}$ \\
\hline Two-finger pinch & $\begin{array}{l}\text { The index finger and middle finger are moved } \\
\text { until they touch the thumb. Similar to the pinch } \\
\text { gesture before, however this has some use as an } \\
\text { alternate select. }\end{array}$ \\
\hline Index Finger Pull & $\begin{array}{l}\text { The palm is facing forward while the hand is } \\
\text { moved side to side at the wrist. This can be } \\
\text { associated with the concept of "good bye", and } \\
\text { ending sequence. }\end{array}$ \\
\hline Good-bye wave & $\begin{array}{l}\text { The palm is facing up with the index finger fully } \\
\text { extended, it is then retracted. This gesture is } \\
\text { usually associated with the "come here" } \\
\text { command. }\end{array}$ \\
\hline
\end{tabular}




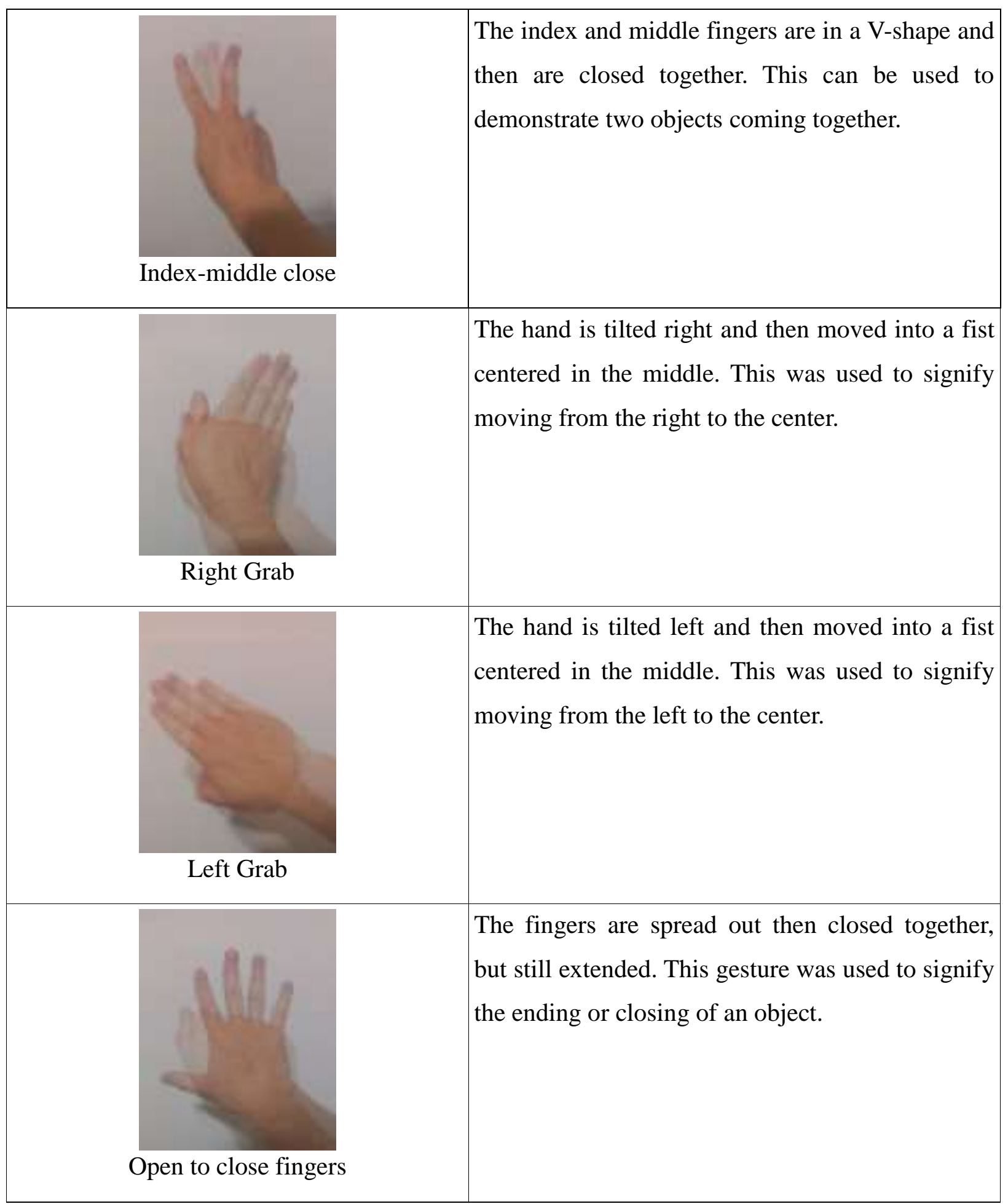




\begin{tabular}{|l|l|}
\hline Spread Hand & $\begin{array}{l}\text { The fingers are closed together, extended and are } \\
\text { then spread out. This gesture was used to signify } \\
\text { the compression. }\end{array}$ \\
\hline Arm side to side & $\begin{array}{l}\text { The wrist and hand are in fixed position while the } \\
\text { arm moves them side to side. This gesture used to } \\
\text { signify the shaking or movement of an object. }\end{array}$ \\
\hline Wrist Tilt & $\begin{array}{l}\text { The hand is in neutral position and is tilted left } \\
\text { and right at the wrist. This object was used to } \\
\text { demonstrate the rotation or axial movement of an } \\
\text { object. }\end{array}$ \\
\hline Deictic & $\begin{array}{l}\text { The hand tilted in the desired direction. This } \\
\text { gesture is used as a command to signify when to } \\
\text { move to the desired direction. The object or } \\
\text { concept is instructed to stop, when the hand tilts to } \\
\text { a direction, it is instructional for the object to } \\
\text { move in that direction. }\end{array}$ \\
\hline & \\
\hline
\end{tabular}




\begin{tabular}{|l|l|}
\hline Fork Tilt & $\begin{array}{l}\text { The index and middle fingers are in a V-shape and } \\
\text { then pointed at a desired direction. }\end{array}$ \\
\hline Palm & $\begin{array}{l}\text { With the fingers extended, the palm is pushed into } \\
\text { the desired direction. Used as a command to push } \\
\text { an object in the desired direction. }\end{array}$ \\
\hline Moving Point & $\begin{array}{l}\text { With the index finger extended, the entire hand is } \\
\text { moved forward in the direction that the finger is } \\
\text { pointing in. }\end{array}$ \\
\hline
\end{tabular}

Table 4.1: Compiled Database Gestures

A table was made separately for the ASL lettering as they were shared similar descriptions. This was used as reference during the experiment. 


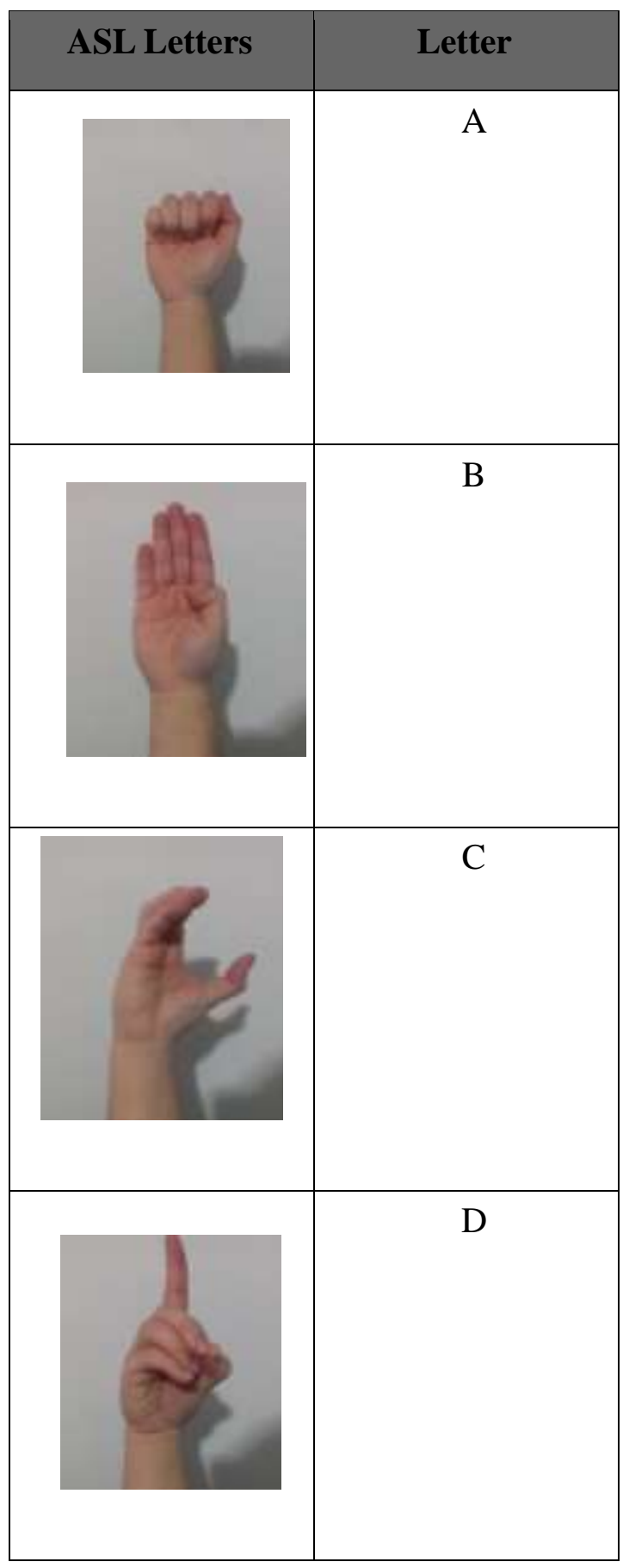




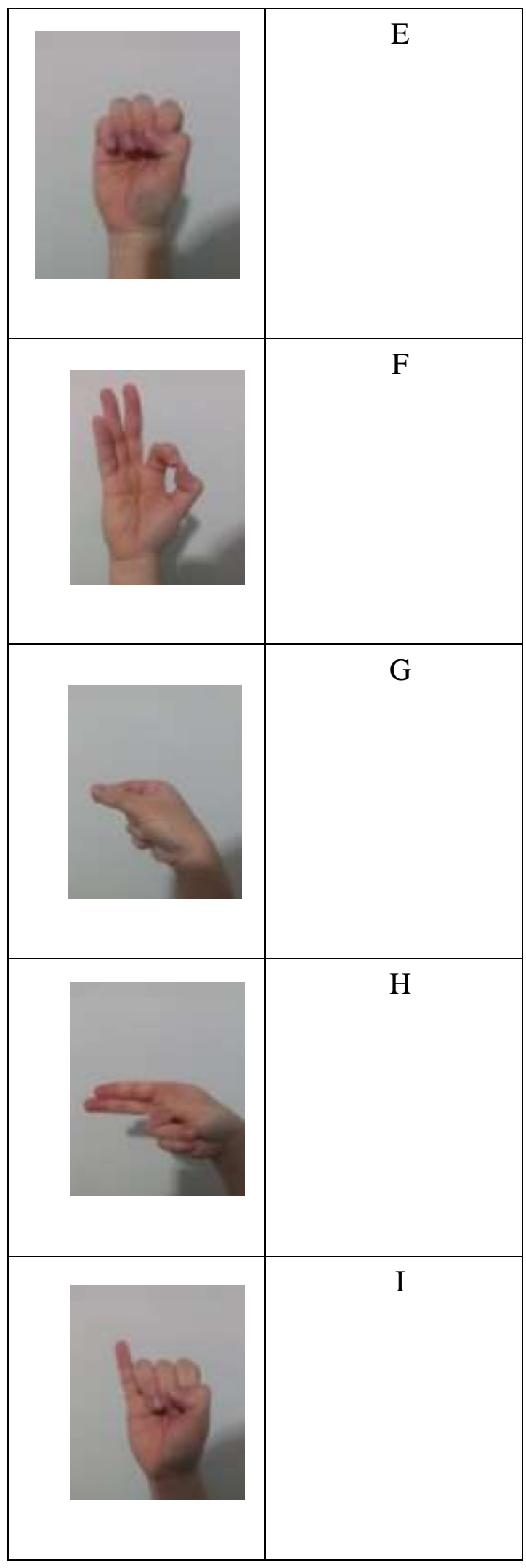

74 


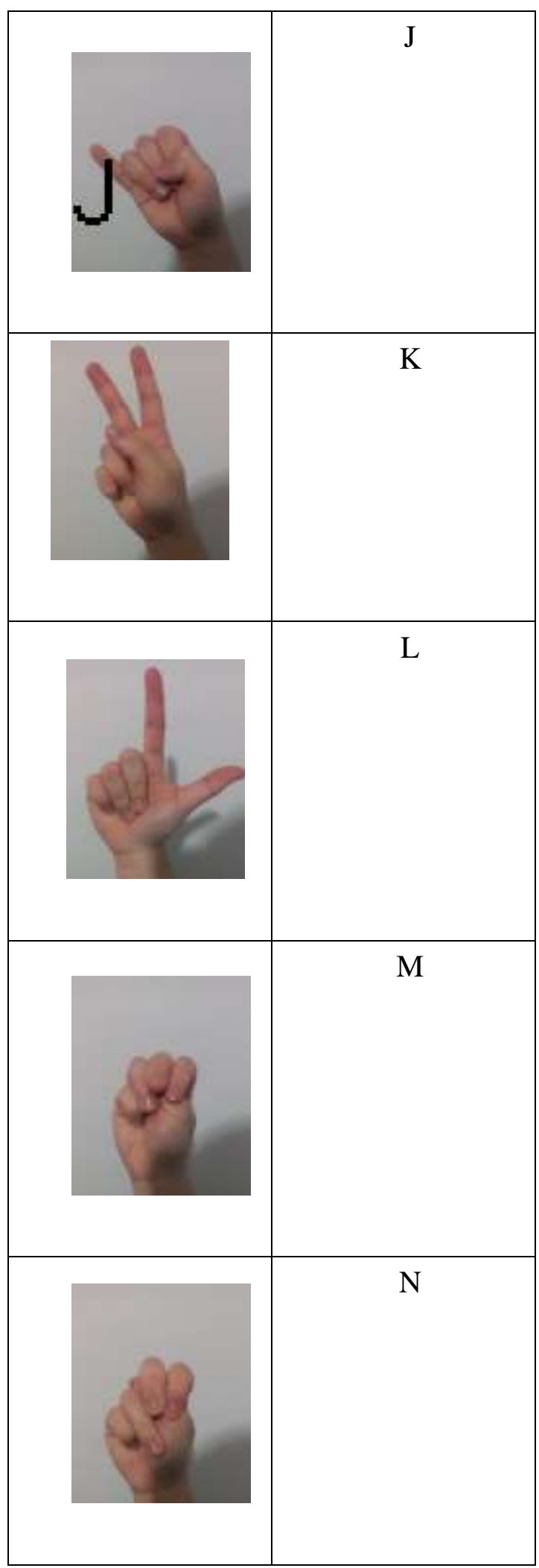




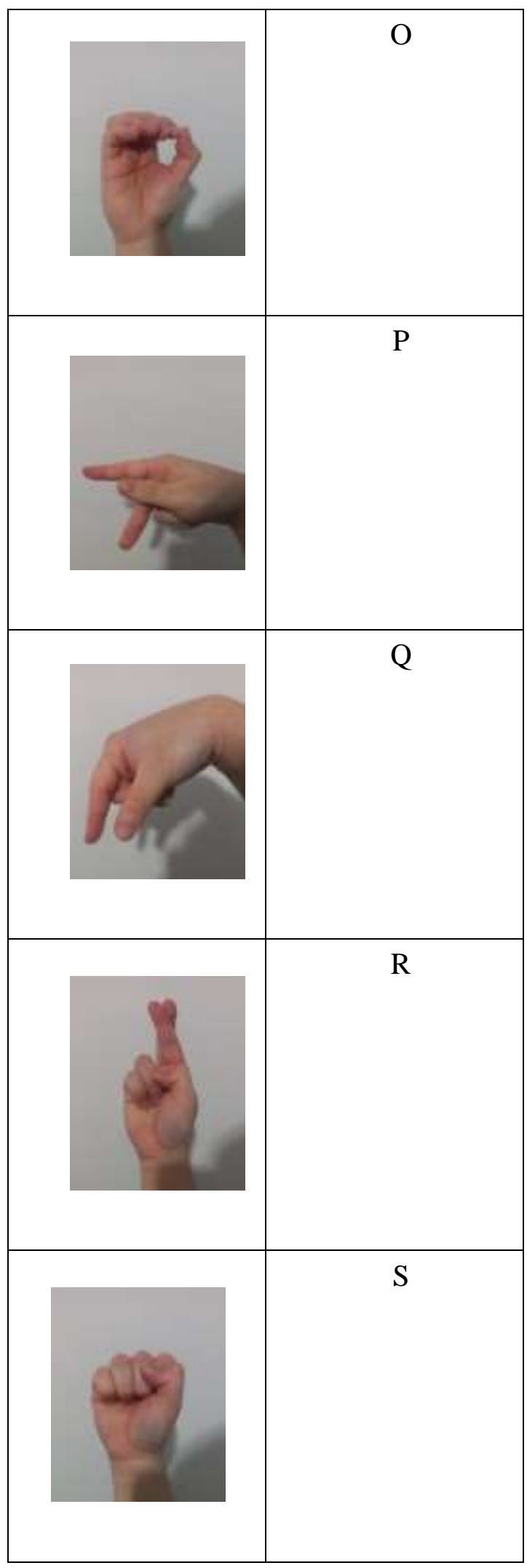

76 


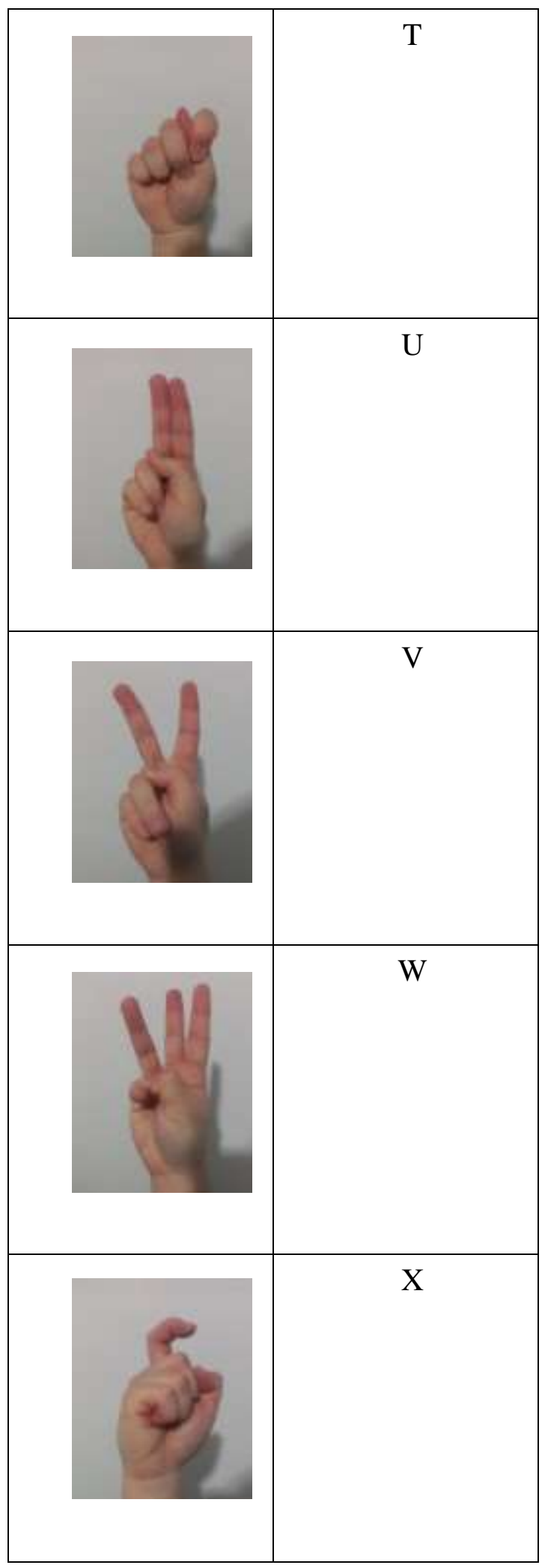




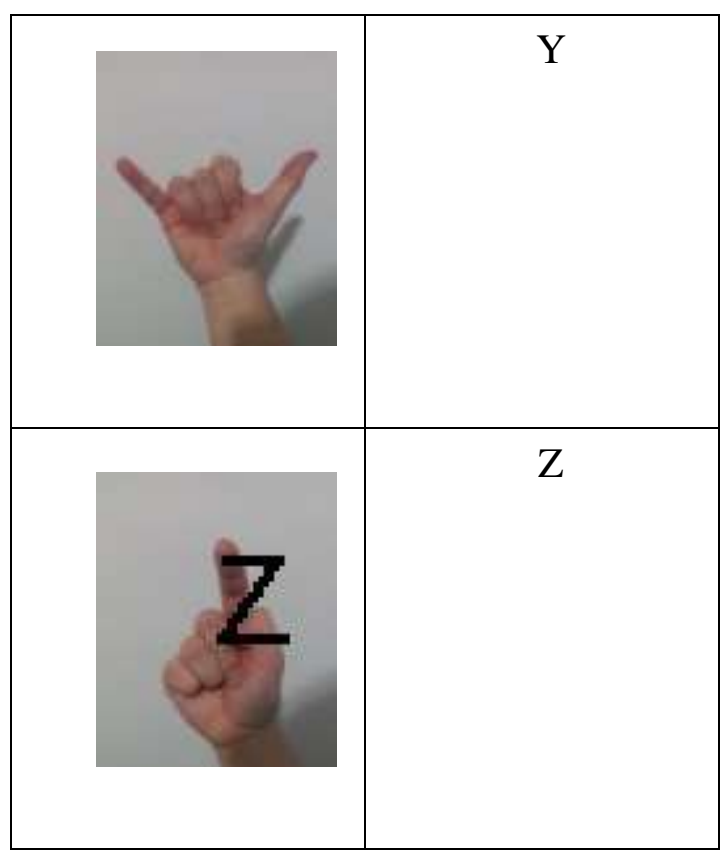

Table 4.2: American Sign Language A-Z 


\section{Appendix 2}

\section{Myo Sensor Data}

In order to get the exertion the user experienced when making a specific gesture, two reference points were required, that is the relaxed state and the exertion state. These were used to compare how stressful the gestures were.

\begin{tabular}{|l|r|r|r|r|l|l|l|r|r|}
\hline Gesture & Pod 1 & Pod 2 & Pod 3 & Pod 4 & Pod 5 & Pod 6 & Pod 7 & Pod 8 & Total \\
\hline Rest & 0 & 0 & 0 & 0 & 0 & 0 & 0 & 0 & 0 \\
\hline Fist & 110 & 70 & 40 & 80 & 40 & 20 & 40 & 60 & 460 \\
\hline
\end{tabular}

Table 3.1: Rest and Exertion Points Reference Table

As with the two reference states above, each gestures was this measured and recorded. 


\begin{tabular}{|c|c|c|c|c|c|c|c|c|c|}
\hline Static: & Pod 1 & Pod 2 & Pod 3 & Pod 4 & Pod 5 & Pod 6 & Pod 7 & Pod 8 & Total \\
\hline \multicolumn{10}{|l|}{ Iconic } \\
\hline ASL A & 80 & 40 & 50 & 40 & 0 & 5 & 0 & 10 & 225 \\
\hline ASL B & 20 & 40 & 80 & 70 & 30 & 20 & 40 & 30 & 330 \\
\hline ASL C & 80 & 50 & 50 & 40 & 10 & 10 & 10 & 10 & 260 \\
\hline ASL D & 20 & 40 & 80 & 80 & 20 & 10 & 40 & 30 & 320 \\
\hline ASLE & 40 & 40 & 90 & 70 & 20 & 20 & 30 & 20 & 330 \\
\hline ASL F & 10 & 20 & 80 & 80 & 10 & 10 & 30 & 10 & 250 \\
\hline ASL G & 20 & 10 & 10 & 20 & 10 & 10 & 10 & 10 & 100 \\
\hline ASL H & 10 & 20 & 40 & 20 & 10 & 10 & 40 & 10 & 160 \\
\hline ASL I & 10 & 50 & 40 & 30 & 20 & 10 & 20 & 20 & 200 \\
\hline ASL K & 30 & 90 & 90 & 90 & 70 & 30 & 30 & 40 & 470 \\
\hline ASL L & 20 & 40 & 40 & 30 & 10 & 20 & 20 & 20 & 200 \\
\hline ASL M & 50 & 80 & 80 & 60 & 20 & 20 & 10 & 30 & 350 \\
\hline ASL N & 20 & 20 & 40 & 20 & 10 & 10 & 10 & 10 & 140 \\
\hline ASLO & 10 & 20 & 40 & 30 & 10 & 10 & 10 & 10 & 140 \\
\hline ASL P & 20 & 10 & 10 & 10 & 10 & 10 & 40 & 20 & 130 \\
\hline ASL Q & 40 & 20 & 20 & 10 & 10 & 10 & 110 & 60 & 280 \\
\hline ASL R & 20 & 40 & 40 & 60 & 20 & 20 & 40 & 30 & 270 \\
\hline ASL S & 50 & 20 & 50 & 20 & 10 & 10 & 10 & 10 & 180 \\
\hline ASL T & 60 & 20 & 40 & 30 & 20 & 20 & 10 & 10 & 210 \\
\hline ASL U & 30 & 40 & 50 & 60 & 30 & 20 & 30 & 20 & 280 \\
\hline ASL V & 20 & 30 & 40 & 60 & 30 & 20 & 30 & 30 & 260 \\
\hline ASL W & 50 & 50 & 70 & 80 & 40 & 30 & 30 & 40 & 390 \\
\hline ASL $X$ & 30 & 20 & 20 & 40 & 10 & 10 & 10 & 20 & 160 \\
\hline ASL Y & 20 & 30 & 40 & 30 & 20 & 20 & 20 & 30 & 210 \\
\hline Stop Gesture & 40 & 50 & 100 & 80 & 40 & 30 & 30 & 20 & 390 \\
\hline Okay Gesture & 20 & 20 & 60 & 40 & 20 & 10 & 30 & 20 & 220 \\
\hline Fingers Closed & 20 & 10 & 30 & 30 & 10 & 10 & 30 & 30 & 170 \\
\hline Thumbs up & 20 & 20 & 30 & 30 & 20 & 10 & 30 & 30 & 190 \\
\hline Thumbs Down & 20 & 10 & 30 & 30 & 10 & 10 & 20 & 20 & 150 \\
\hline \multicolumn{10}{|l|}{ Deictic } \\
\hline Point & 20 & 20 & 30 & 20 & 10 & 10 & 20 & 30 & 160 \\
\hline Hand point & 20 & 20 & 40 & 30 & 10 & 10 & 20 & 20 & 170 \\
\hline Two-Finger Point & 20 & 20 & 40 & 40 & 10 & 10 & 20 & 20 & 180 \\
\hline
\end{tabular}

Table 4.1: Myo Armband sensor Readings Static (Activity Levels) 


\begin{tabular}{|c|c|c|c|c|c|c|c|c|}
\hline Gesture & Pod 1 & Pod 2 & Pod 3 & Pod 4 & Pod 5 & Pod 6 & Pod 7 & Pod 8 \\
\hline Rest & 0.00 & 0.00 & 0.00 & 0.00 & 0.00 & 0.00 & 0.00 & 0.00 \\
\hline Fist & 1.00 & 0.64 & 0.36 & 0.73 & 0.36 & 0.18 & 0.36 & 0.55 \\
\hline \multicolumn{9}{|l|}{ Static: } \\
\hline \multicolumn{9}{|l|}{ Iconic } \\
\hline$\overline{A S L A}$ & 0.73 & 0.36 & 0.45 & 0.36 & 0.00 & 0.05 & 0.00 & 0.09 \\
\hline ASL B & 0.18 & 0.36 & 0.73 & 0.64 & 0.27 & 0.18 & 0.36 & 0.27 \\
\hline ASL C & 0.73 & 0.45 & 0.45 & 0.36 & 0.09 & 0.09 & 0.09 & 0.09 \\
\hline$\overline{A S L D}$ & 0.18 & 0.36 & 0.73 & 0.73 & 0.18 & 0.09 & 0.36 & 0.27 \\
\hline ASL E & 0.36 & 0.36 & 0.82 & 0.64 & 0.18 & 0.18 & 0.27 & 0.18 \\
\hline ASL F & 0.09 & 0.18 & 0.73 & 0.73 & 0.09 & 0.09 & 0.27 & 0.09 \\
\hline ASL G & 0.18 & 0.09 & 0.09 & 0.18 & 0.09 & 0.09 & 0.09 & 0.09 \\
\hline$\overline{A S L ~ H}$ & 0.09 & 0.18 & 0.36 & 0.18 & 0.09 & 0.09 & 0.36 & 0.09 \\
\hline ASL I & 0.09 & 0.45 & 0.36 & 0.27 & 0.18 & 0.09 & 0.18 & 0.18 \\
\hline ASL K & 0.27 & 0.82 & 0.82 & 0.82 & 0.64 & 0.27 & 0.27 & 0.36 \\
\hline ASL L & 0.18 & 0.36 & 0.36 & 0.27 & 0.09 & 0.18 & 0.18 & 0.18 \\
\hline$\overline{A S L} M$ & 0.45 & 0.73 & 0.73 & 0.55 & 0.18 & 0.18 & 0.09 & 0.27 \\
\hline ASL N & 0.18 & 0.18 & 0.36 & 0.18 & 0.09 & 0.09 & 0.09 & 0.09 \\
\hline ASL O & 0.09 & 0.18 & 0.36 & 0.27 & 0.09 & 0.09 & 0.09 & 0.09 \\
\hline ASL P & 0.18 & 0.09 & 0.09 & 0.09 & 0.09 & 0.09 & 0.36 & 0.18 \\
\hline$\overline{A S L Q}$ & 0.36 & 0.18 & 0.18 & 0.09 & 0.09 & 0.09 & 1.00 & 0.55 \\
\hline ASL R & 0.18 & 0.36 & 0.36 & 0.55 & 0.18 & 0.18 & 0.36 & 0.27 \\
\hline ASL S & 0.45 & 0.18 & 0.45 & 0.18 & 0.09 & 0.09 & 0.09 & 0.09 \\
\hline ASL T & 0.55 & 0.18 & 0.36 & 0.27 & 0.18 & 0.18 & 0.09 & 0.09 \\
\hline$\overline{A S L U}$ & 0.27 & 0.36 & 0.45 & 0.55 & 0.27 & 0.18 & 0.27 & 0.18 \\
\hline ASL V & 0.18 & 0.27 & 0.36 & 0.55 & 0.27 & 0.18 & 0.27 & 0.27 \\
\hline ASL W & 0.45 & 0.45 & 0.64 & 0.73 & 0.36 & 0.27 & 0.27 & 0.36 \\
\hline ASL X & 0.27 & 0.18 & 0.18 & 0.36 & 0.09 & 0.09 & 0.09 & 0.18 \\
\hline$\overline{A S L} Y$ & 0.18 & 0.27 & 0.36 & 0.27 & 0.18 & 0.18 & 0.18 & 0.27 \\
\hline Stop Gesture & 0.36 & 0.45 & 0.91 & 0.73 & 0.36 & 0.27 & 0.27 & 0.18 \\
\hline Okay Gesture & 0.18 & 0.18 & 0.55 & 0.36 & 0.18 & 0.09 & 0.27 & 0.18 \\
\hline Fingers Closed & 0.18 & 0.09 & 0.27 & 0.27 & 0.09 & 0.09 & 0.27 & 0.27 \\
\hline Thumbs up & 0.18 & 0.18 & 0.27 & 0.27 & 0.18 & 0.09 & 0.27 & 0.27 \\
\hline Thumbs Down & 0.18 & 0.09 & 0.27 & 0.27 & 0.09 & 0.09 & 0.18 & 0.18 \\
\hline \multicolumn{9}{|l|}{ Deictic } \\
\hline Point & 0.18 & 0.18 & 0.27 & 0.18 & 0.09 & 0.09 & 0.18 & 0.27 \\
\hline Hand point & 0.18 & 0.18 & 0.36 & 0.27 & 0.09 & 0.09 & 0.18 & 0.18 \\
\hline Two-Finger Point & 0.18 & 0.18 & 0.36 & 0.36 & 0.09 & 0.09 & 0.18 & 0.18 \\
\hline
\end{tabular}

Table 4.2: Static Gesture Myo Armband Readings Normalized 


\begin{tabular}{|c|c|c|c|c|c|c|c|c|c|}
\hline Dynamic: & Pod 1 & Pod 2 & Pod 3 & Pod 4 & Pod 5 & Pod 6 & Pod 7 & Pod 8 & Total \\
\hline \multicolumn{10}{|l|}{ Iconic } \\
\hline Circular Right & 40 & 60 & 50 & 40 & 20 & 20 & 30 & 30 & 290 \\
\hline Circular Left & 30 & 50 & 50 & 30 & 20 & 10 & 30 & 30 & 250 \\
\hline Dial Control & 20 & 40 & 40 & 30 & 20 & 20 & 20 & 20 & 210 \\
\hline Lever Control & 20 & 30 & 30 & 30 & 20 & 20 & 20 & 10 & 180 \\
\hline Wave Right & 40 & 70 & 100 & 100 & 50 & 40 & 30 & 30 & 460 \\
\hline Wave Left & 60 & 20 & 40 & 20 & 20 & 20 & 80 & 40 & 300 \\
\hline Wave Up & 10 & 10 & 30 & 30 & 10 & 10 & 40 & 20 & 160 \\
\hline Wave Down & 50 & 30 & 30 & 20 & 10 & 10 & 50 & 40 & 240 \\
\hline Lever Spin & 20 & 40 & 40 & 30 & 10 & 10 & 10 & 10 & 170 \\
\hline Lever Pull & 10 & 20 & 30 & 30 & 10 & 10 & 10 & 10 & 130 \\
\hline Switch & 40 & 30 & 30 & 20 & 10 & 10 & 20 & 20 & 180 \\
\hline Pinch & 20 & 30 & 30 & 40 & 20 & 10 & 10 & 20 & 180 \\
\hline Spread Pinch & 20 & 20 & 20 & 20 & 10 & 10 & 10 & 20 & 130 \\
\hline Grab & 90 & 80 & 80 & 80 & 30 & 20 & 30 & 50 & 460 \\
\hline Press down & 40 & 40 & 40 & 30 & 20 & 10 & 40 & 30 & 250 \\
\hline Lift Up & 40 & 30 & 50 & 40 & 20 & 10 & 30 & 30 & 250 \\
\hline Arm Rotate & 20 & 50 & 30 & 30 & 20 & 20 & 10 & 10 & 190 \\
\hline Knocking & 60 & 60 & 50 & 50 & 20 & 20 & 40 & 40 & 340 \\
\hline Two-finger pinch & 20 & 30 & 40 & 30 & 20 & 20 & 10 & 10 & 180 \\
\hline Index Finger Pull & 20 & 10 & 10 & 20 & 20 & 10 & 30 & 10 & 130 \\
\hline ASL J & 10 & 10 & 20 & 20 & 10 & 10 & 20 & 20 & 120 \\
\hline ASL Z & 20 & 40 & 30 & 20 & 10 & 10 & 20 & 20 & 170 \\
\hline \multicolumn{10}{|l|}{ Metaphoric } \\
\hline Good-bye wave & 20 & 40 & 40 & 40 & 40 & 20 & 20 & 20 & 240 \\
\hline Index-middle close & 20 & 20 & 40 & 40 & 20 & 20 & 30 & 30 & 220 \\
\hline Right Grab & 40 & 40 & 50 & 40 & 20 & 20 & 20 & 30 & 260 \\
\hline Left Grab & 30 & 40 & 40 & 40 & 20 & 10 & 40 & 30 & 250 \\
\hline Open to close fingers & 40 & 30 & 70 & 50 & 20 & 20 & 30 & 30 & 290 \\
\hline Spread Hand & 70 & 40 & 70 & 50 & 30 & 20 & 20 & 30 & 330 \\
\hline Arm side to side & 60 & 60 & 70 & 40 & 20 & 20 & 10 & 20 & 300 \\
\hline Wrist Tilt & 30 & 40 & 30 & 40 & 20 & 20 & 20 & 10 & 210 \\
\hline \multicolumn{10}{|l|}{ Deictic } \\
\hline Tilt & 30 & 20 & 20 & 30 & 20 & 20 & 20 & 40 & 200 \\
\hline Fork Tilt & 30 & 20 & 40 & 40 & 30 & 20 & 30 & 30 & 240 \\
\hline Palm & 20 & 30 & 40 & 40 & 30 & 20 & 20 & 10 & 210 \\
\hline \multirow[t]{2}{*}{ Moving Point } & 20 & 20 & 20 & 20 & 10 & 10 & 20 & 30 & 150 \\
\hline & 25 & 22.5 & 30 & 32.5 & 22.5 & 17.5 & 22.5 & 27.5 & 200 \\
\hline
\end{tabular}

Table 4.3: Myo Armband sensor Readings Dynamic 


\begin{tabular}{|c|c|c|c|c|c|c|c|c|}
\hline Dynamic: & & & & & & & & \\
\hline Iconic & & & & & & & & \\
\hline Circular Right & 0.36 & 0.55 & 0.45 & 0.36 & 0.18 & 0.18 & 0.27 & 0.27 \\
\hline Circular Left & 0.27 & 0.45 & 0.45 & 0.27 & 0.18 & 0.09 & 0.27 & 0.27 \\
\hline Dial Control & 0.18 & 0.36 & 0.36 & 0.27 & 0.18 & 0.18 & 0.18 & 0.18 \\
\hline Lever Control & 0.18 & 0.27 & 0.27 & 0.27 & 0.18 & 0.18 & 0.18 & 0.09 \\
\hline Wave Right & 0.36 & 0.64 & 0.91 & 0.91 & 0.45 & 0.36 & 0.27 & 0.27 \\
\hline Wave Left & 0.55 & 0.18 & 0.36 & 0.18 & 0.18 & 0.18 & 0.73 & 0.36 \\
\hline Wave Up & 0.09 & 0.09 & 0.27 & 0.27 & 0.09 & 0.09 & 0.36 & 0.18 \\
\hline Wave Down & 0.45 & 0.27 & 0.27 & 0.18 & 0.09 & 0.09 & 0.45 & 0.36 \\
\hline Lever Spin & 0.18 & 0.36 & 0.36 & 0.27 & 0.09 & 0.09 & 0.09 & 0.09 \\
\hline Lever Pull & 0.09 & 0.18 & 0.27 & 0.27 & 0.09 & 0.09 & 0.09 & 0.09 \\
\hline Switch & 0.36 & 0.27 & 0.27 & 0.18 & 0.09 & 0.09 & 0.18 & 0.18 \\
\hline Pinch & 0.18 & 0.27 & 0.27 & 0.36 & 0.18 & 0.09 & 0.09 & 0.18 \\
\hline Spread Pinch & 0.18 & 0.18 & 0.18 & 0.18 & 0.09 & 0.09 & 0.09 & 0.18 \\
\hline Grab & 0.82 & 0.73 & 0.73 & 0.73 & 0.27 & 0.18 & 0.27 & 0.45 \\
\hline Press down & 0.36 & 0.36 & 0.36 & 0.27 & 0.18 & 0.09 & 0.36 & 0.27 \\
\hline Lift Up & 0.36 & 0.27 & 0.45 & 0.36 & 0.18 & 0.09 & 0.27 & 0.27 \\
\hline Arm Rotate & 0.18 & 0.45 & 0.27 & 0.27 & 0.18 & 0.18 & 0.09 & 0.09 \\
\hline Knocking & 0.55 & 0.55 & 0.45 & 0.45 & 0.18 & 0.18 & 0.36 & 0.36 \\
\hline Two-finger pinch & 0.18 & 0.27 & 0.36 & 0.27 & 0.18 & 0.18 & 0.09 & 0.09 \\
\hline Index Finger Pull & 0.18 & 0.09 & 0.09 & 0.18 & 0.18 & 0.09 & 0.27 & 0.09 \\
\hline ASL J & 0.09 & 0.09 & 0.18 & 0.18 & 0.09 & 0.09 & 0.18 & 0.18 \\
\hline ASL Z & 0.18 & 0.36 & 0.27 & 0.18 & 0.09 & 0.09 & 0.18 & 0.18 \\
\hline \begin{tabular}{|l} 
Metaphoric \\
\end{tabular} & & & & & & & & \\
\hline Good-bye wave & 0.18 & 0.36 & 0.36 & 0.36 & 0.36 & 0.18 & 0.18 & 0.18 \\
\hline Index-middle close & 0.18 & 0.18 & 0.36 & 0.36 & 0.18 & 0.18 & 0.27 & 0.27 \\
\hline Right Grab & 0.36 & 0.36 & 0.45 & 0.36 & 0.18 & 0.18 & 0.18 & 0.27 \\
\hline Left Grab & 0.27 & 0.36 & 0.36 & 0.36 & 0.18 & 0.09 & 0.36 & 0.27 \\
\hline Open to close fingers & 0.36 & 0.27 & 0.64 & 0.45 & 0.18 & 0.18 & 0.27 & 0.27 \\
\hline Spread Hand & 0.64 & 0.36 & 0.64 & 0.45 & 0.27 & 0.18 & 0.18 & 0.27 \\
\hline Arm side to side & 0.55 & 0.55 & 0.64 & 0.36 & 0.18 & 0.18 & 0.09 & 0.18 \\
\hline Wrist Tilt & 0.27 & 0.36 & 0.27 & 0.36 & 0.18 & 0.18 & 0.18 & 0.09 \\
\hline Deictic & & & & & & & & \\
\hline Tilt & 0.27 & 0.18 & 0.18 & 0.27 & 0.18 & 0.18 & 0.18 & 0.36 \\
\hline Fork Tilt & 0.27 & 0.18 & 0.36 & 0.36 & 0.27 & 0.18 & 0.27 & 0.27 \\
\hline Palm & 0.18 & 0.27 & 0.36 & 0.36 & 0.27 & 0.18 & 0.18 & 0.09 \\
\hline Moving Point & 0.18 & 0.18 & 0.18 & 0.18 & 0.09 & 0.09 & 0.18 & 0.27 \\
\hline
\end{tabular}

Table 4.4: Dynamic Gesture Myo Armband Readings Normalized 
A program was used to graphically plot the data for later review.

\begin{tabular}{|c|c|}
\hline Myo Sensor Readings & Description \\
\hline 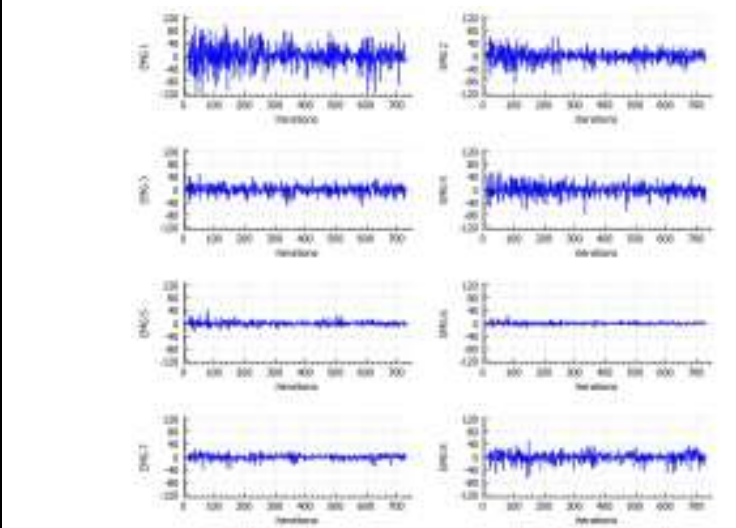 & Clenched Fist \\
\hline $\begin{array}{ll} & \\
& \end{array}$ & Moving to Rest \\
\hline 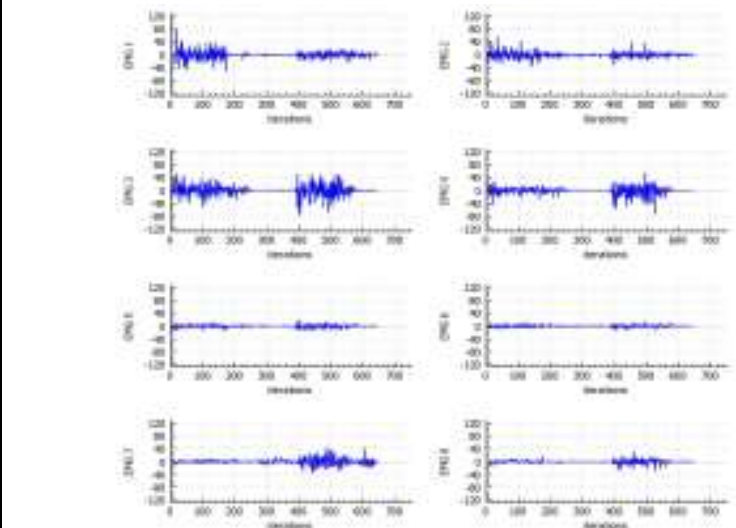 & $\begin{array}{l}\text { ASL A } \\
\text { ASL B }\end{array}$ \\
\hline
\end{tabular}




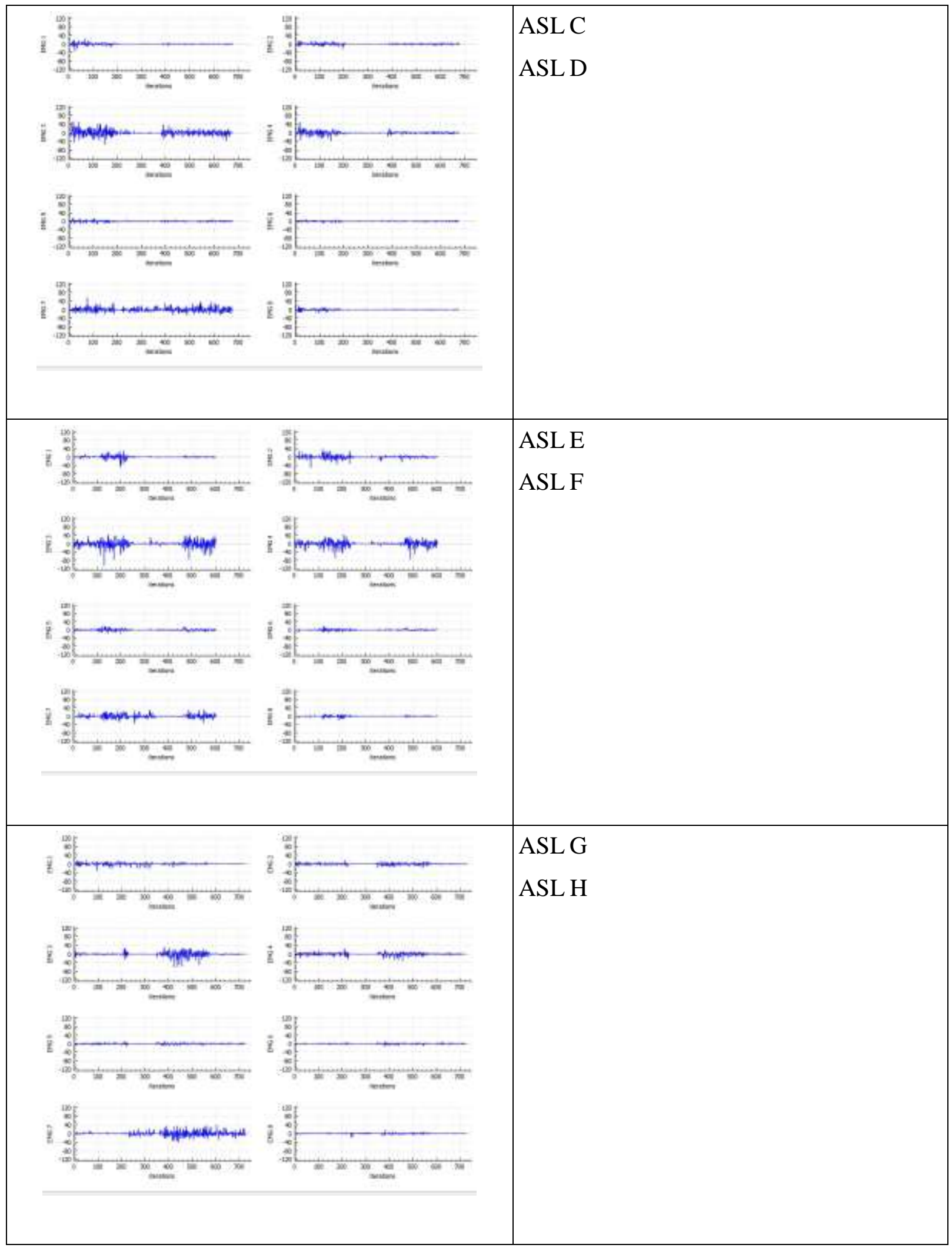




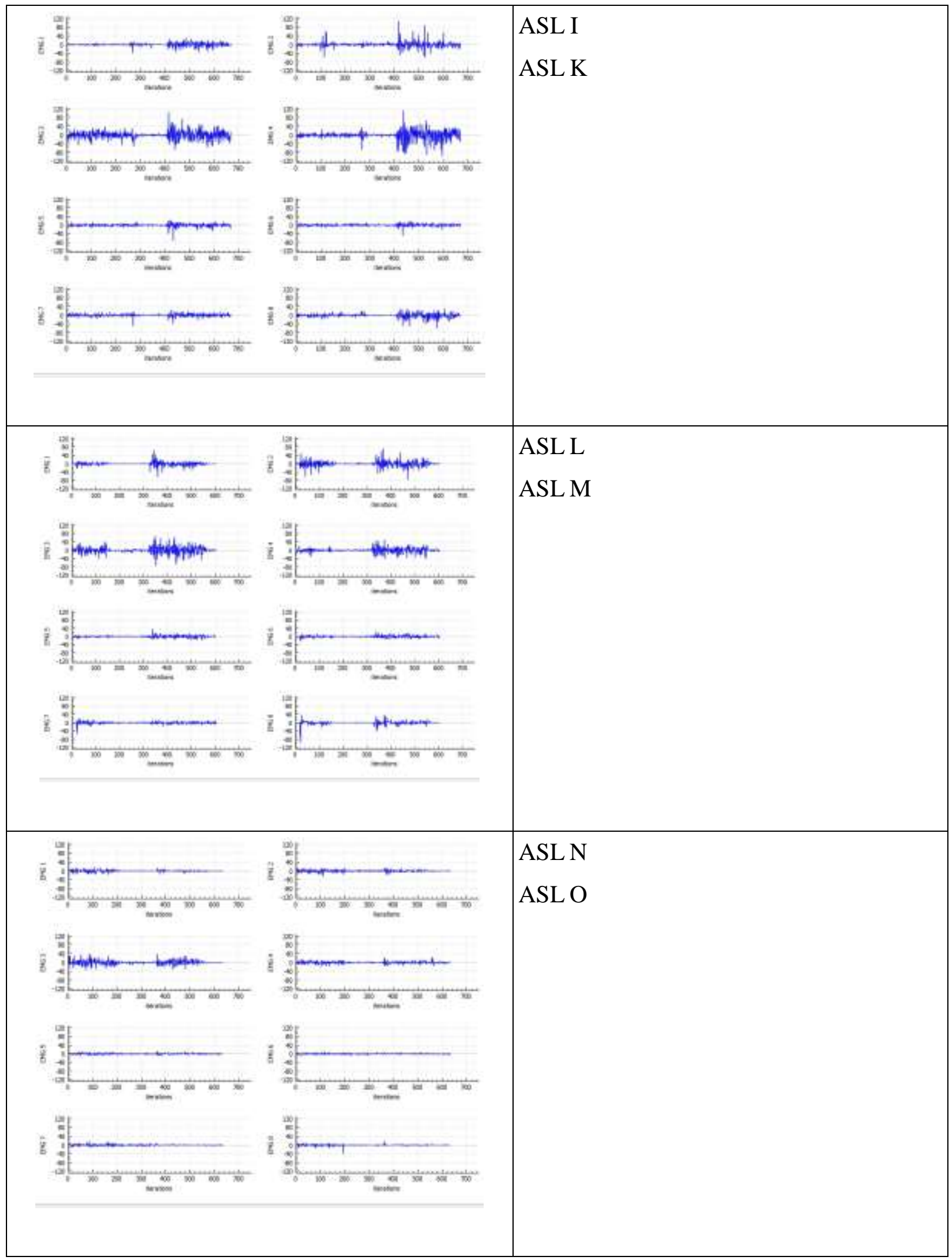




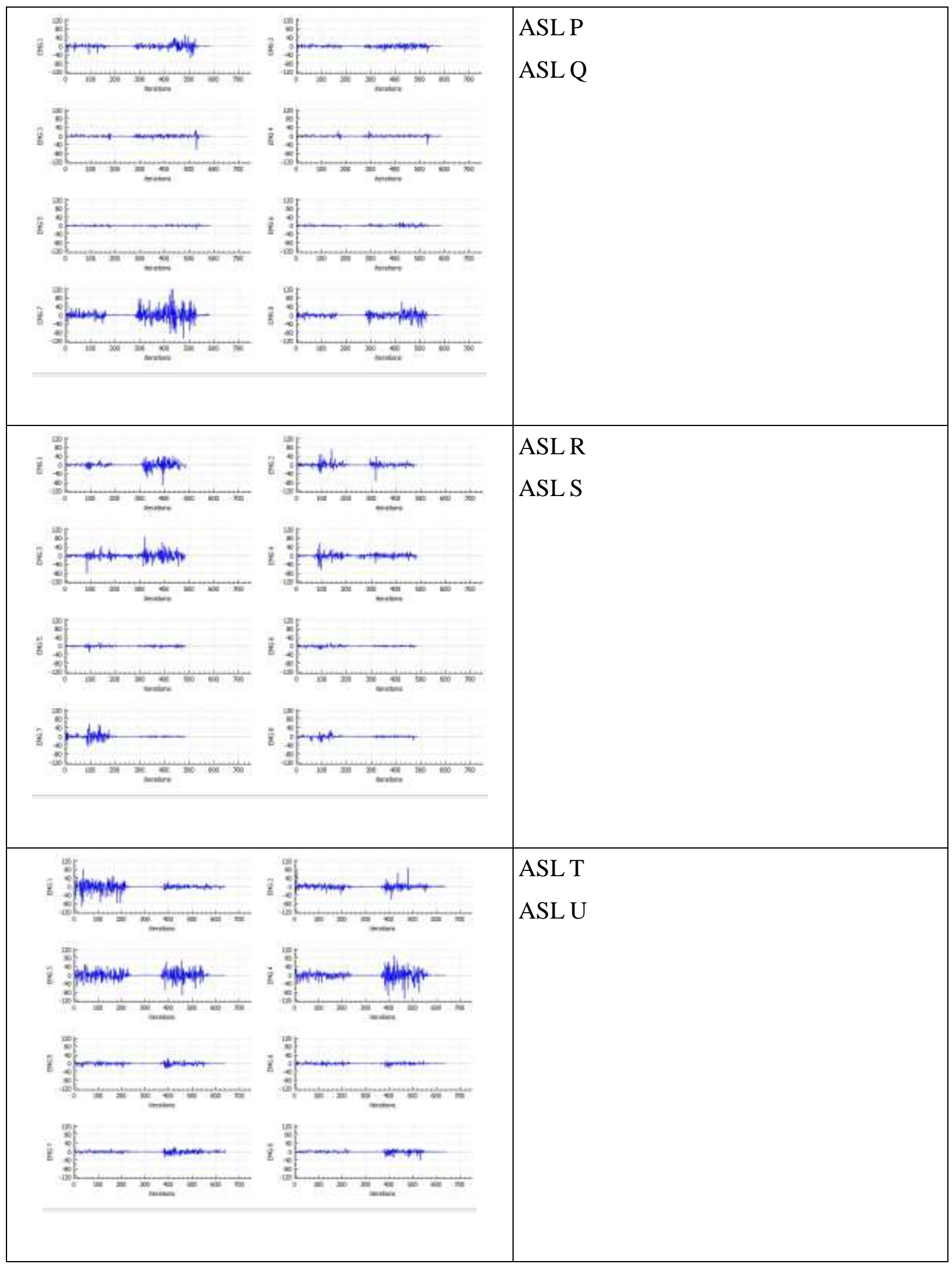




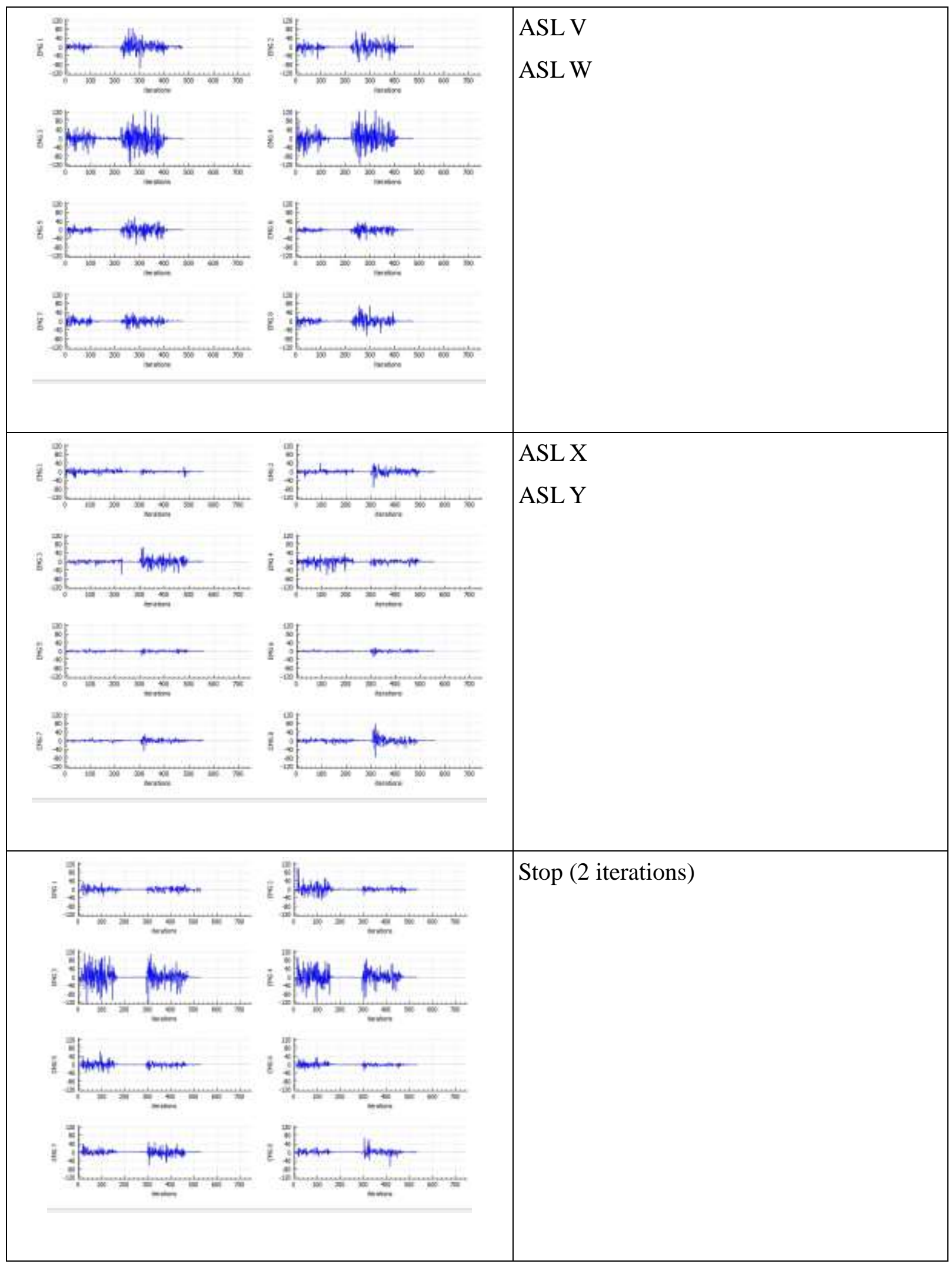




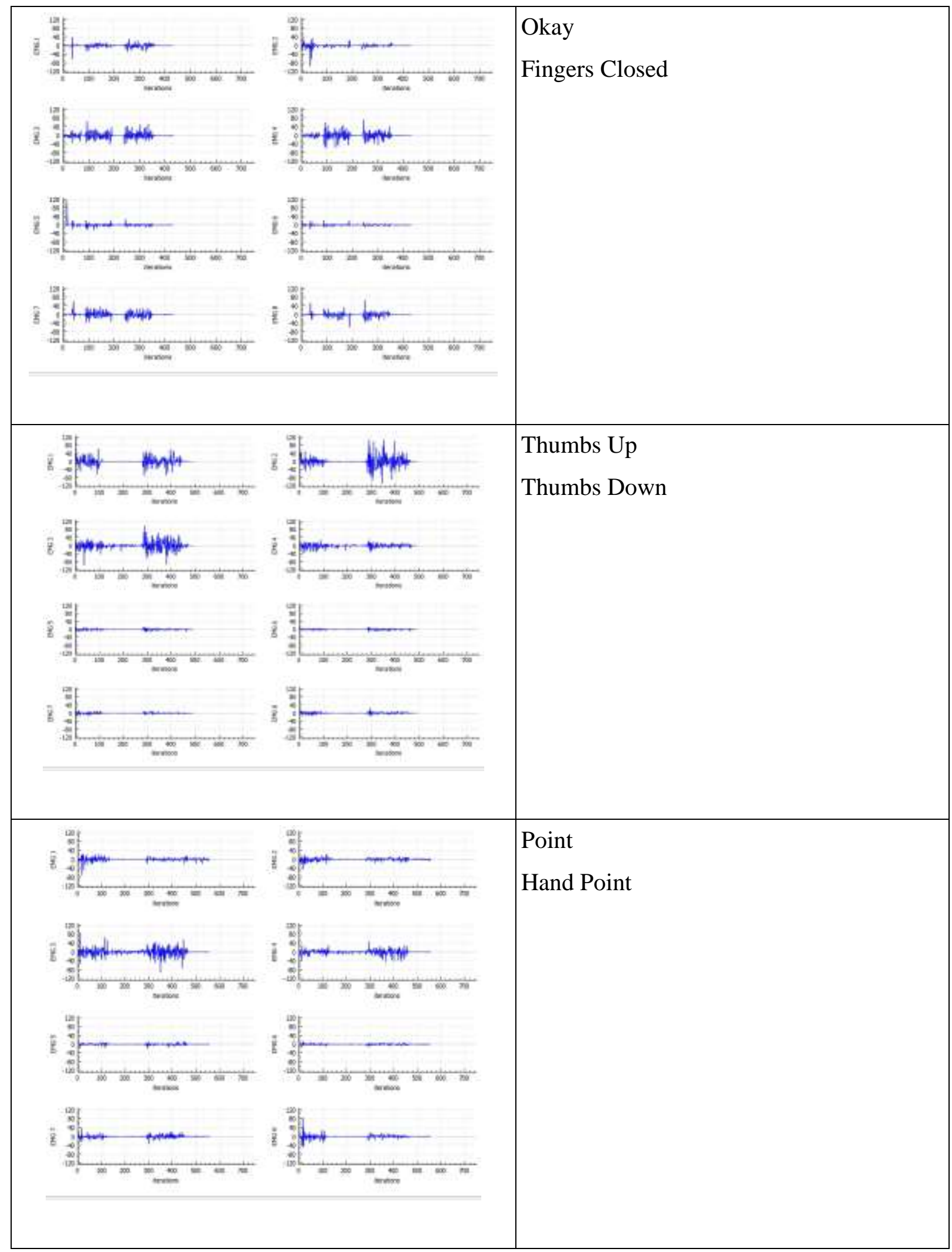




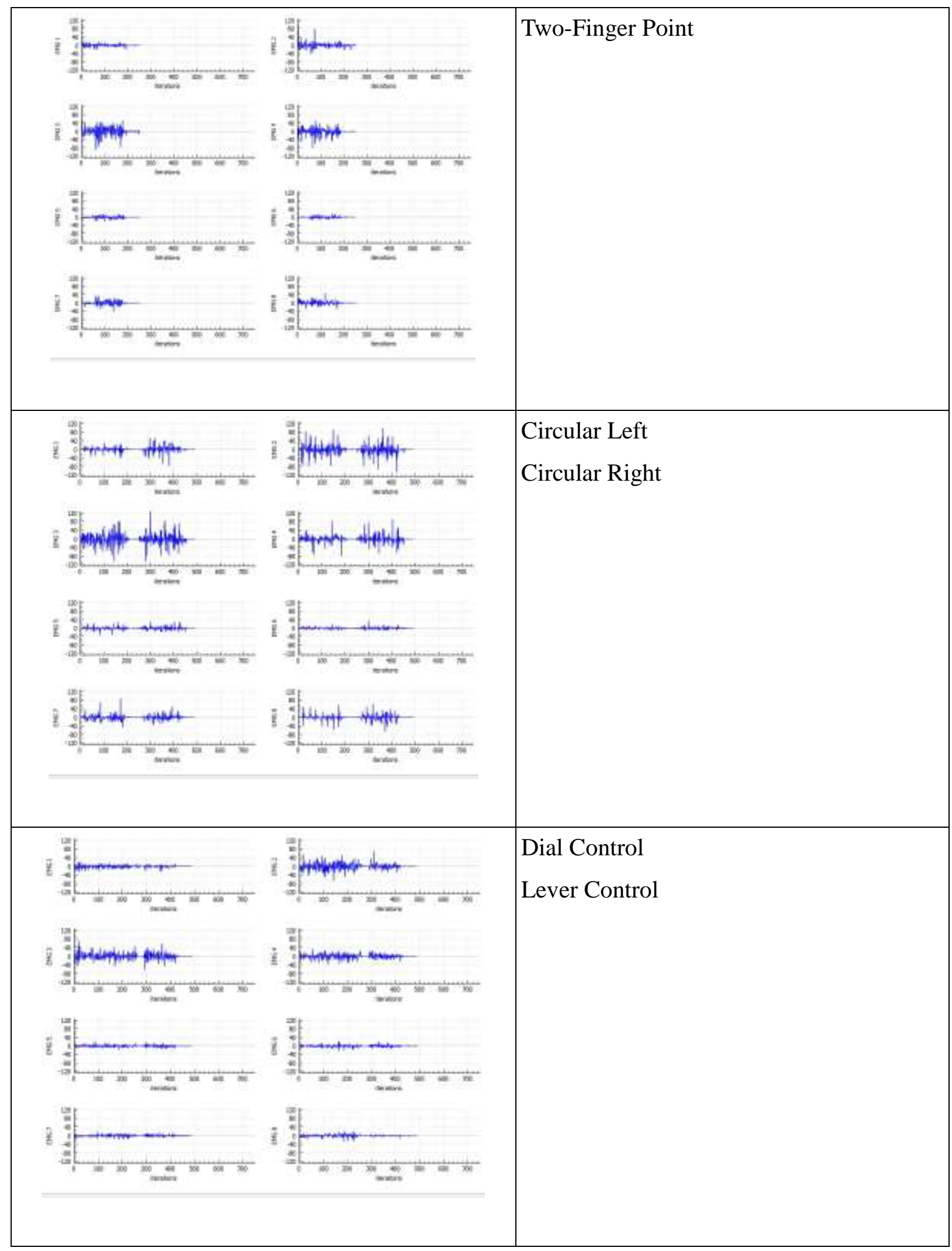




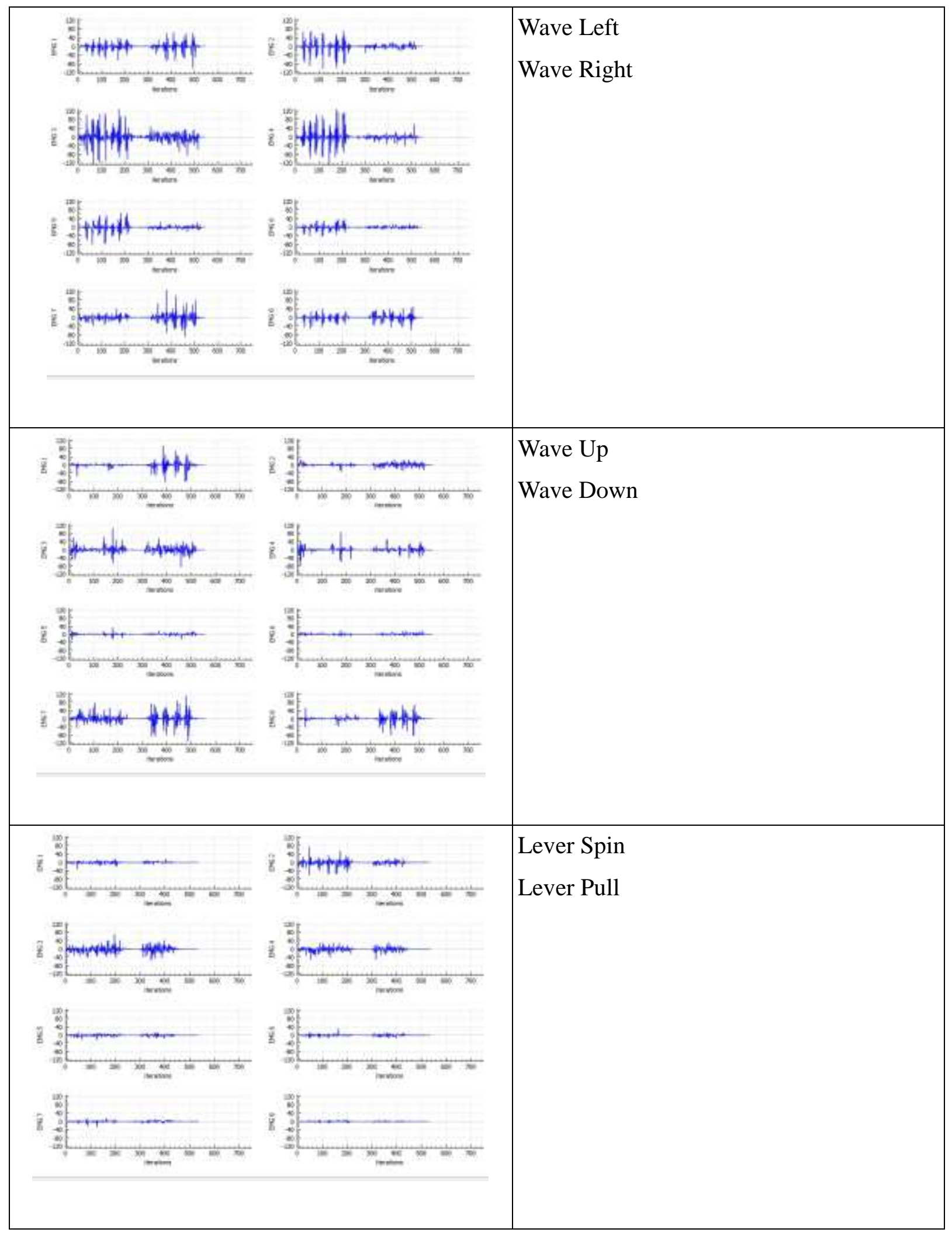




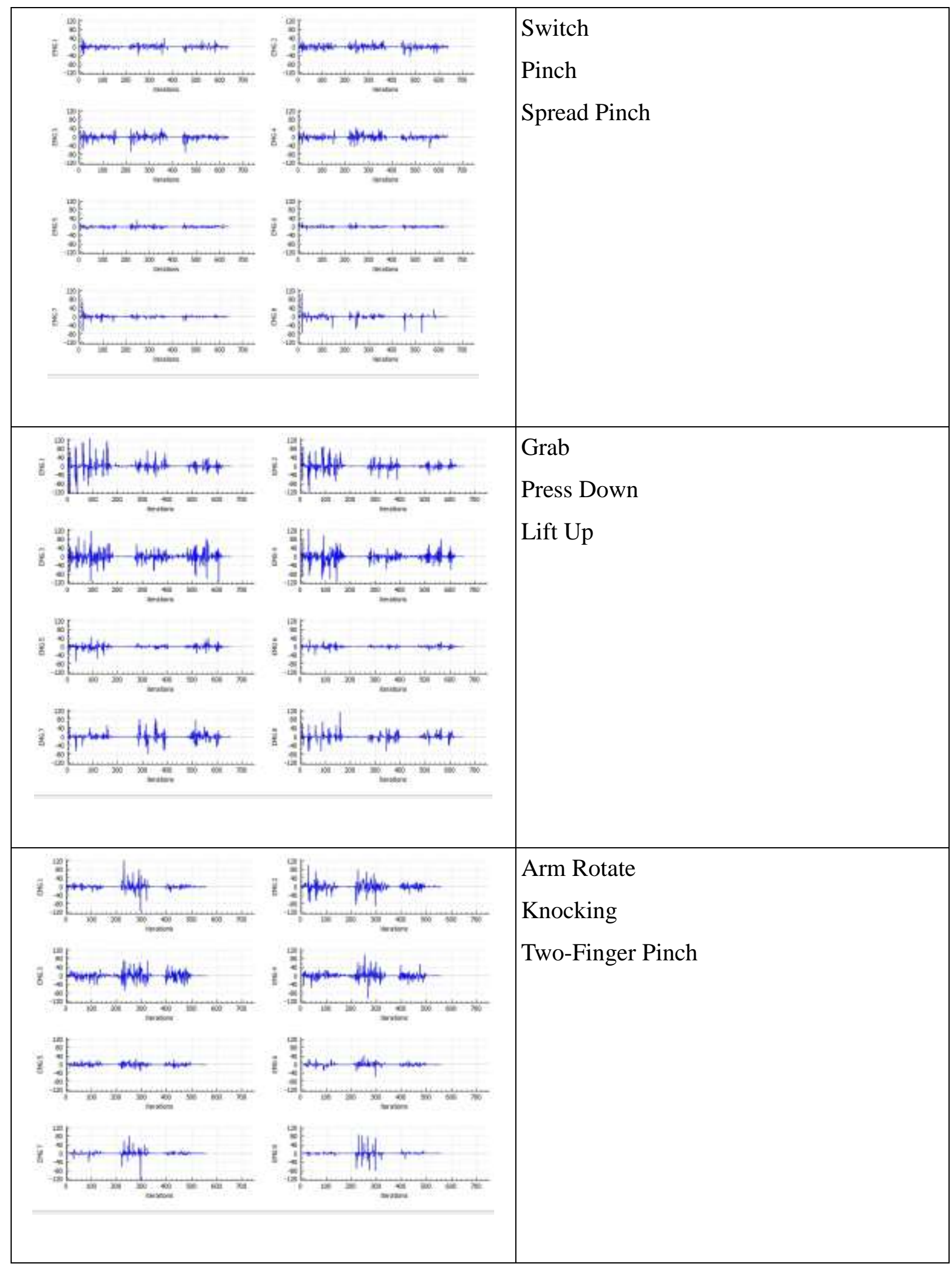




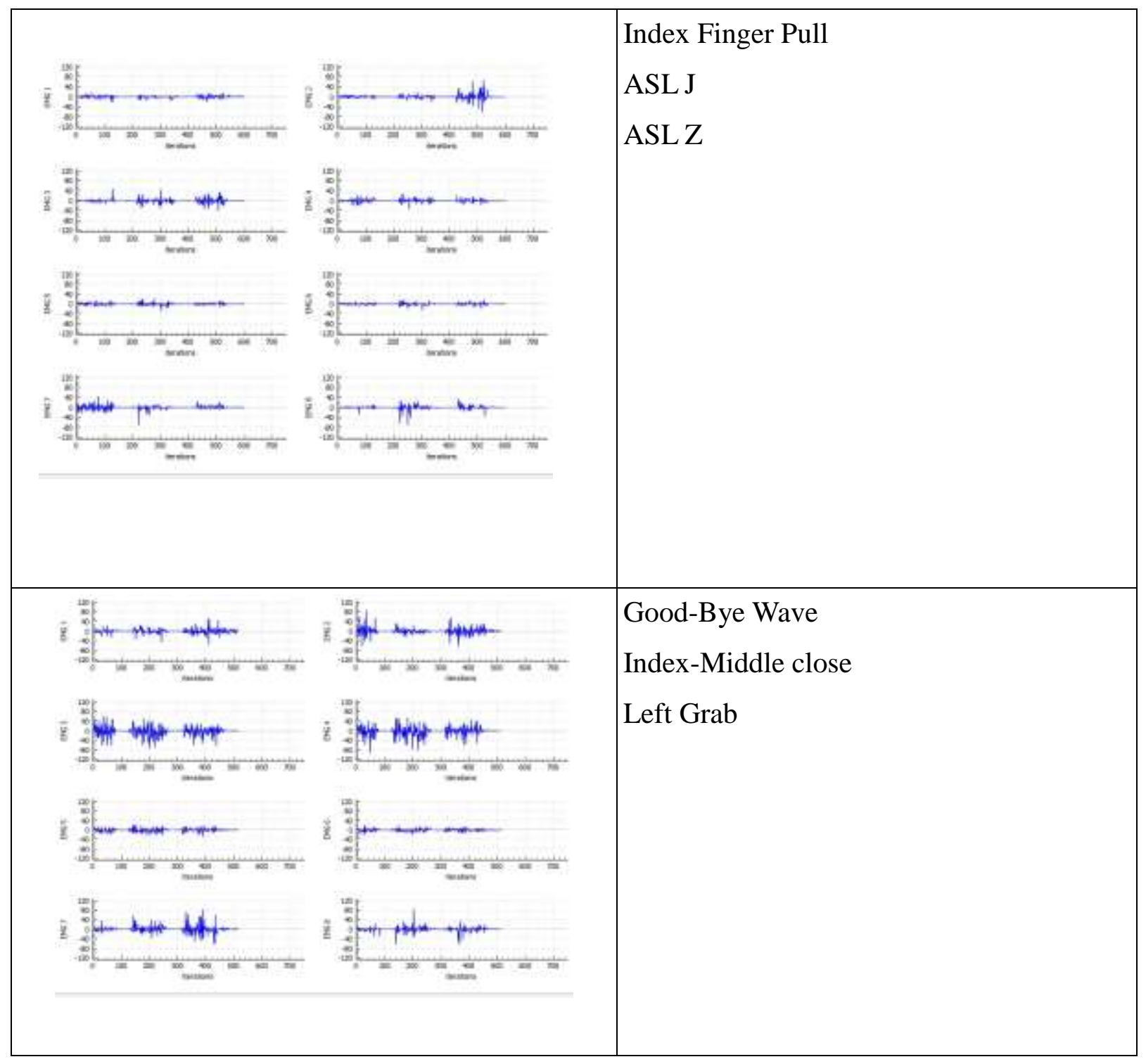




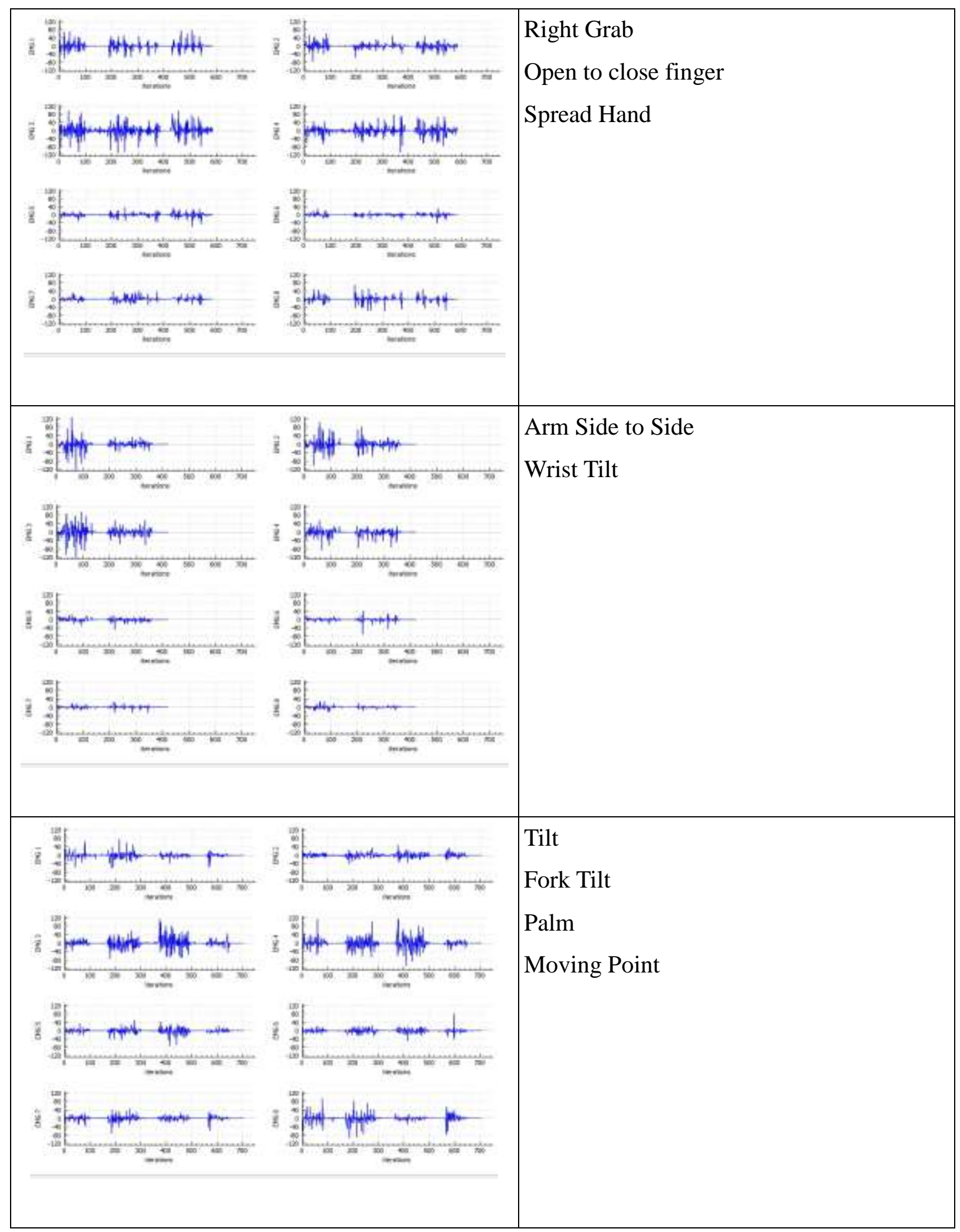

Table 4.5: Myo Armband EMG Plots 


\section{Appendix 3}

\section{Gesture Movement Rating}

The hand was measured using the upper most limits and the lowest the fingers can compress as reference points. Once that was recorded, each finger was measured in reference to the limits. This was done because the limits and possible degree can vary from person to person, by using the subject's limits; it is possible to create comparisons with respect to exertion.

\begin{tabular}{|l|c|c|c|c|c|c|c|}
\hline Gesture & Thumb Axis & Thumb Flex & Index & Middle & Ring & Little & Wrist \\
\hline Rest & 0 & 0 & 0 & 0 & 0 & 0 & 0 \\
\hline Fist & -10 & -10 & -10 & -10 & -10 & -10 & 0 \\
\hline Extended & 10 & 10 & 10 & 10 & 10 & 10 & 0 \\
\hline
\end{tabular}

Table 3.2: Rest, flex and clenched reference table 


\begin{tabular}{|c|c|c|c|c|c|c|c|}
\hline Static: & Thumb Axis & Thumb Flex & Index & Middle & Ring & Little & Wrist \\
\hline \multicolumn{8}{|l|}{ Iconic } \\
\hline ASL A & -8 & 0 & -8 & -8 & -8 & -8 & 0 \\
\hline ASL B & -10 & 10 & 10 & 10 & 10 & 10 & 0 \\
\hline ASL C & 0 & 0 & 1 & 2 & 1 & 1 & 0 \\
\hline ASL D & 0 & 0 & 10 & -3 & -4 & -5 & 0 \\
\hline ASLE & 0 & 5 & 7 & 7 & 7 & 7 & 0 \\
\hline ASL F & 0 & 4 & -1 & 8 & 8 & 8 & 0 \\
\hline ASL G & 0 & 0 & 0 & -10 & -10 & -10 & 3 \\
\hline ASL H & -10 & 10 & 10 & 10 & -10 & -10 & 4 \\
\hline ASL I & -10 & 10 & -10 & -10 & -8 & 10 & 0 \\
\hline ASL K & -5 & 8 & 10 & 10 & -10 & -7 & 0 \\
\hline ASL L & -10 & 10 & 10 & -10 & -10 & -10 & 0 \\
\hline ASL M & -10 & 0 & -6 & -6 & -6 & -10 & 0 \\
\hline ASL N & -10 & 0 & -6 & -6 & -10 & -10 & 0 \\
\hline ASLO & 0 & 0 & -1 & -1 & -1 & -1 & 0 \\
\hline ASL P & 10 & 4 & 10 & 10 & -10 & -10 & 8 \\
\hline ASL Q & 0 & 5 & 7 & -10 & -10 & -10 & 10 \\
\hline ASL R & -10 & -10 & 10 & 10 & -10 & -10 & 0 \\
\hline ASL S & -10 & -10 & -10 & -10 & -10 & -10 & 0 \\
\hline ASL T & -10 & 0 & 6 & -10 & -10 & -10 & 0 \\
\hline ASL U & -4 & 0 & 10 & 10 & -10 & -10 & 0 \\
\hline ASL V & -4 & 0 & 10 & 10 & -10 & -10 & 0 \\
\hline ASL W & -10 & -10 & 10 & 10 & 10 & -10 & 0 \\
\hline ASL X & 0 & -7 & 5 & -10 & -10 & -10 & 0 \\
\hline ASL Y & 10 & 10 & -10 & -10 & -10 & 10 & 0 \\
\hline Stop Gesture & -4 & -7 & 10 & 10 & 10 & 10 & 0 \\
\hline Okay Gesture & 0 & 0 & -2 & 10 & 10 & 10 & 0 \\
\hline Fingers Closed & 0 & -7 & 10 & 10 & 10 & 10 & 0 \\
\hline Thumbs up & 10 & 10 & -10 & -10 & -10 & -10 & 0 \\
\hline Thumbs Down & 10 & 10 & -10 & -10 & -10 & -10 & 0 \\
\hline \multicolumn{8}{|l|}{ Deictic } \\
\hline Point & -10 & -10 & 10 & -10 & -10 & -10 & 0 \\
\hline Hand point & 10 & 10 & 10 & 10 & 10 & 10 & 0 \\
\hline Two-Finger Point & -10 & -10 & 10 & 10 & -10 & -10 & 0 \\
\hline
\end{tabular}

Table 4.6: Movement Range Table Static

With the reference points established, the process of measuring and recording the positioning with respect to the reference points. 


\begin{tabular}{|c|c|c|c|c|c|c|c|}
\hline Dynamic: & Thumb Axis & Thumb Flex & Index & Middle & Ring & Little & Wrist \\
\hline \multicolumn{8}{|l|}{ Iconic } \\
\hline Circular Right & 0 & -7 & 10 & 10 & 10 & 10 & 10 \\
\hline Circular Left & 0 & -7 & 10 & 10 & 10 & 10 & 10 \\
\hline Dial Control & 3 & 0 & -3 & -10 & -10 & -10 & 10 \\
\hline Lever Control & 1 & 1 & 1 & 1 & 1 & 1 & 0 \\
\hline Wave Right & 0 & 0 & 10 & 10 & 10 & 10 & 10 \\
\hline Wave Left & 0 & 0 & 10 & 10 & 10 & 10 & 10 \\
\hline Wave Up & 0 & 0 & 10 & 10 & 10 & 10 & 10 \\
\hline Wave Down & 0 & 0 & 10 & 10 & 10 & 10 & 10 \\
\hline Lever Spin & 1 & 1 & 1 & 1 & 1 & 1 & 10 \\
\hline Lever Pull & 1 & 1 & 1 & 1 & 1 & 1 & 0 \\
\hline Switch & -10 & -10 & 10 & -10 & -10 & -10 & 0 \\
\hline Pinch & 3 & 0 & -3 & -10 & -10 & -10 & 0 \\
\hline Spread Pinch & 3 & 0 & -3 & -10 & -10 & -10 & 0 \\
\hline Grab & -10 & -10 & -10 & -10 & -10 & -10 & 0 \\
\hline Press down & 0 & -7 & 10 & 10 & 10 & 10 & 0 \\
\hline Lift Up & 0 & -7 & 10 & 10 & 10 & 10 & 0 \\
\hline Arm Rotate & -10 & -10 & -10 & -10 & -10 & -10 & 10 \\
\hline Knocking & -10 & -10 & -10 & -10 & -10 & -10 & 10 \\
\hline Two-finger pinch & -5 & -5 & -5 & -5 & -10 & -10 & 0 \\
\hline Index Finger Pull & -10 & -10 & -10 & -10 & -10 & -10 & 0 \\
\hline ASL J & 10 & 10 & -10 & -10 & -8 & 10 & 8 \\
\hline ASL Z & -5 & -10 & 7 & -10 & -10 & -10 & 0 \\
\hline \multicolumn{8}{|l|}{ Metaphoric } \\
\hline Good-bye wave & 10 & 10 & 10 & 10 & 10 & 10 & 10 \\
\hline Index-middle close & -10 & -10 & 10 & 10 & -10 & -10 & 0 \\
\hline Right Grab & -10 & -10 & -10 & -10 & -10 & -10 & 8 \\
\hline Left Grab & -10 & -10 & -10 & -10 & -10 & -10 & 8 \\
\hline Open to close fingers & 10 & 10 & 10 & 10 & 10 & 10 & 0 \\
\hline Spread Hand & 10 & 10 & 10 & 10 & 10 & 10 & 0 \\
\hline Arm side to side & -10 & -10 & -10 & -10 & -10 & -10 & -10 \\
\hline Wrist Tilt & 0 & 0 & 0 & 0 & 0 & 0 & 10 \\
\hline \multicolumn{8}{|l|}{ Deictic } \\
\hline Tilt & 0 & 0 & 5 & 5 & 5 & 5 & 10 \\
\hline Fork Tilt & -10 & -10 & 10 & 10 & -10 & -10 & 10 \\
\hline Palm & 0 & 0 & 10 & 10 & 10 & 10 & 10 \\
\hline Moving Point & -10 & -10 & 10 & -10 & -10 & -10 & 10 \\
\hline
\end{tabular}

Table 4.7: Movement Range Table Dynamic 


\section{References}

[1]H. Duan, Q. Zhang and W. Ma, 'An approach to dynamic hand gesture modeling and realtime extraction', in 2011 IEEE 3rd International Conference on Communication Software and Networks (ICCSN), Xi'an, 2011, pp. 139-142.

[2]J. Gallud, P. Villanueva, R. Tesoriero, G. Sabastian, S. Molina and A. Navarret, 'GestureBased Interaction: Concept Map and Application Scenarios', in 2010 Third International Conference on Advances in Human-Oriented and Personalized Mechanisms, Technologies and Services (CENTRIC), Nice, 2010, pp. 28-33.

[3]L. Gupta and S. Ma, 'Gesture-based interaction and communication: automated classification of hand gesture contours', inSystems, Man, and Cybernetics, Part C: Applications and Reviews, IEEE Transactions on (Volume:31 , Issue: 1 ), IEEE, 2001, pp. 114-120.

[4]M. Malgireddy, J. Corso, V. Govindaraju and D. Mandalapu, 'A Framework for Hand Gesture Recognition and Spotting Using Sub-gesture Modeling', in 2010 20th International Conference on Pattern Recognition (ICPR), Istanbul, 2010, pp. 3780-3783.

[5]M. Panwar, 'Hand gesture recognition based on shape parameters', in 2012 International Conference on Computing, Communication and Applications (ICCCA), Dindigul, Tamilnadu, 2012, pp. 1-6.

[6]H. Tauseef, M. Fahiem and S. Forhan, 'Browse Conference Publications > Visualisation, 2009. VIZ '09. ... Help Working with Abstracts Back to Results Recognition and Translation of Hand Gestures to Urdu Alphabets Using a Geometrical Classification', in Visualisation, 2009. VIZ '09. Second International Conference, Barcelona, 2009, pp. 213-217.

[7]S. Jambhale and A. Khaparde, 'Gesture recognition using DTW \& piecewise DTW', in Electronics and Communication Systems (ICECS), 2014 International Conference, Coimbatore, 2014, pp. 1-5.

[8]M. Neggazi, M. Bengherabi, Z. Boulkenafet and A. Amira, 'An efficient FPGA implementation of Gaussian mixture models based classifier: Application to face recognition', in 2013 8th International Workshop on Systems, Signal Processing and their Applications (WoSSPA), Algiers, 2013, pp. 367 - 371. 
[9]F. Jiang, Y. Gao, S. Wu, D. Zhao and C. Wang, 'Discriminating features learning in hand gesture classification', IET Computer Vision, vol. 9, no. 5, pp. 673-680, 2015.

[10]F. Erden and A. Çetin, 'Hand gesture based remote control system using infrared sensors and a camera', IEEE Transactions on Consumer Electronics, vol. 60, no. 4, pp. 675-680, 2014.

[11]M. Kaâniche and F. Brémond, 'Recognizing Gestures by Learning Local Motion Signatures of HOG Descriptors', IEEE Transactions on Pattern Analysis and Machine Intelligence, vol. 34, no. 11, pp. 2247-2258, 2012.

[12] J. Gallud, P. Villanueva, R. Tesoriero, G. Sebastian, S. Molina and A. Navarrete, "GestureBased Interaction: Concept Map and Application Scenarios", in Advances in Human-Oriented and Personalized Mechanisms, Technologies and Services (CENTRIC), 2010 Third International Conference, Nice, 2010, pp. 28-33.

[13] J. Lothian Murray, R. Linden and D. Kendall, Sociology in our times. Toronto: Nelson Education, 2012.

[14] J. Oates and A. Grayson, Cognitive and language development in children. Milton Keynes, U.K.: Open University, 2004.

[15]M. Roetting and O. Zukunft, "Don't touch that tablet: An evaluation of gaze-based interfaces for tablet computers", in 2014 6th International Conference on Mobile Computing, Applications and Services (MobiCASE), TX, 2014, pp. $49-56$.

[16]V. Reddy, V. Raghuveer, J. Krishna and K. Chandralohit, "Finger gesture based tablet interface", in2012 IEEE International Conference on Computational Intelligence \& Computing Research (ICCIC), Coimbatore, 2012, pp. $1-4$.

[17]N. Conci, P. Ceresato and F. De Natale, "Natural Human-Machine Interface using an Interactive Virtual Blackboard", in IEEE International Conference on Image Processing, 2007 ICIP 2007, San Antonio, TX, 2007, pp. V - 181 - V - 184.

[18]K. Ouwehand, T. van Gog and F. Paas, "Effects of Gestures on Older Adults' Learning from Video-based Models", Applied Cognitive Psychology, vol. 29, no. 1, pp. 115-128, 2014.

[19]K. Liebal, T. Behne, M. Carpenter and M. Tomasello, "Infants use shared experience to interpret pointing gestures", Developmental Science, vol. 12, no. 2, pp. 264-271, 2009.

[20]Y. Shen, S. Ong and A. Nee, "Vision-Based Hand Interaction in Augmented Reality Environment",International Journal of Human-Computer Interaction, vol. 27, no. 6, pp. 523544, 2011.

[21]D. Rempel, M. Camilleri and D. Lee, "The design of hand gestures for human-computer 
interaction: Lessons from sign language interpreters", International Journal of HumanComputer Studies, vol. 72, no. 10-11, pp. 728-735, 2014.

[22]J. Lambrecht, H. Walzel and J. Kruger, "Robust finger gesture recognition on handheld devices for spatial programming of industrial robots", in RO-MAN, 2013 IEEE, Gyeongju, 2013, pp. $99-106$.

[23]X. Meng, C. Cheung, K. Ho, K. Lui, E. Lam and V. Tam, "Building smart cameras on mobile tablets for hand gesture recognition", in 2012 Sixth International Conference on Distributed Smart Cameras (ICDSC), Hong Kong, 2012, pp. 1 - 5.

[24]W. Hurst, R. Poppe and J. Angeren, "Drawing outside the lines: Tracking-based gesture interaction in mobile augmented entertainment", in 2015 7th International Conference on Intelligent Technologies for Interactive Entertainment (INTETAIN), Turin, 2015, pp. 79 - 87.

[25]P. Treffner, M. Peter and M. Kleidon, "Gestures and Phases: The Dynamics of Speech-Hand Communication", Ecological Psychology, vol. 20, no. 1, pp. 32-64, 2008.

[26]J. Vauclair and J. Imbault, "Relationship between manual preferences for object manipulation and pointing gestures in infants and toddlers", Developmental Science, vol. 12, no. 6, pp. 1060-1069, 2009.

[27]J. Graham and S. Heywood, "The effects of elimination of hand gestures and of verbal codability on speech performance", European Journal of Social Psychology, vol. 5, no. 2, pp. 189-195, 1975.

[28]Y. Lin, H. Chen, Y. Hsieh, M. Hu and W. Cheng, "Interactive human action search using body language", in Wireless and Optical Communications Conference (WOCC), 201221 st Annual, Kaohsiung, 2012, pp. 30 - 31.

[29]Z. Ren, J. Meng and J. Yuan, "Depth camera based hand gesture recognition and its applications in Human-Computer-Interaction", in 2011 8th International Conference on Information, Communications and Signal Processing (ICICS), Singapore, 2011, pp. 1 - 5.

[30]V. Bhame, R. Sreemathy and H. Dhumal, "Vision based hand gesture recognition using eccentric approach for human computer interaction", in 2014 International Conference on Advances in Computing, Communications and Informatics (ICACCI), New Delhi, 2014, pp. 949 $-953$.

[31]J. Jong, J. Hong and C. Yen, "Persistence temperament associated with children playing math games between touch panel and embodied interaction", Journal of Computer Assisted Learning, vol. 29, no. 6, pp. 569-578, 2013.

[32]A. Pereira, "Computer input devices: Design for well-being and productivity", Ph.D, 
University of California, Berkeley, US, 2015.

[33]S. Billinghurst and K. Vu, "Touch screen gestures for web browsing tasks", Computers in Human Behavior, vol. 53, pp. 71-81, 2015.

[34]H. Ogata, Y. Sakamoto, T. Takei and A. Torige, "Hand Shape Discrimination in Touch Interface toward Personal Authentication on a Tablet Computer", in 2013 International Conference on Biometrics and Kansei Engineering, Tokyo, 2013, pp. 257 - 260.

[35]H. Elluech, A. Wali and A. Alimi, "Smart Tablet Monitoring by a Real-Time Head Movement and Eye Gestures Recognition System", in 2014 International Conference on Future Internet of Things and Cloud, Barcelona, 2014, pp. $393-398$.

[36]K. Deshmane and S. Ghodake, "Android Software Based Multi-touch Gestures Recognition for Secure Biometric Modality", in 2015 International Conference on Information Technology (ICIT), Bhubaneswar, 2015, pp. 107 - 112.

[37]N. Aziz, "Children's Interaction with Tablet Applications: Gestures and Interface Design", in International Journal of Computer and Information Technology, Loughborough, 2013, pp. 447-450.

[38]J. Liang, Y. Han and Q. Hu, "Semi-supervised image clustering with multi-modal information", Multimedia Systems, vol. 22, no. 2, pp. 149-160, 2014.

[39]D. Ghosh and S. Ari, "On an algorithm for Vision-based hand gesture recognition", Signal, Image and Video Processing, vol. 10, no. 4, pp. 655-662, 2015.

[40]R. Ahmed, M. El Sayed, S. Gadsden, J. Tjong and S. Habibi, "Artificial neural network training utilizing the smooth variable structure filter estimation strategy", Neural Computer \& Applications, vol. 27, no. 3, pp. 537-548, 2015.x

[41]Y. Zhou, G. Jiang and Y. Lin, "A novel finger and hand pose estimation technique for realtime hand gesture recognition", Pattern Recognition, vol. 49, pp. 102-114, 2016.

[42]Y. Fuh and H. Ho, "Highly flexible self-powered sensors based on printed circuit board technology for human motion detection and gesture recognition", Nanotechnology, vol. 27, no. 9, p. 095401, 2016.

[43]F. Mod Ma'asum, S. Sulaiman and A. Saparon, "An Overview of Hand Gestures Recognition System Techniques", IOP Conf. Ser:: Mater. Sci. Eng., vol. 99, p. 012012, 2015.

[44]E. Saykol, H. Türe, A. Sirvanci and M. Turan, "Posture labeling based gesture classification for Turkish sign language using depth values", Kybernetes, vol. 45, no. 4, pp. 604-621, 2016. 
[45]S. Smithies, K. Novins and J. Arvo, "Equation entry and editing via handwriting and gesture recognition", Behaviour \& Information Technology, vol. 20, no. 1, pp. 53-67, 2001.

[46]M. Sun, X. Ren, H. Tu and F. Tian, "An Investigation Into the Relationship Between Texture and Human Performance in Steering and Gesture Input Tasks", International Journal of HumanComputer Interaction, vol. 30, no. 8, pp. 654-662, 2014.

[47]D. Rozado, T. Moreno, J. San Agustin, F. Rodriguez and P. Varona, "Controlling a Smartphone Using Gaze Gestures as the Input Mechanismx", Human-Computer Interaction, vol. 30, no. 1, pp. 34-63, 2014.

[48]H. Stern, J. Wachs and Y. Edan, "Optimal Hand Gesture Vocabulary Design Using PsychoPhysiological and Technical Factors", 7th International Conference on Automatic Face and Gesture Recognition (FGR06).

[49]H. Stern, J. Wachs and Y. Edan, "Hand gesture vocabulary design: a multicriteria optimization",2004 IEEE International Conference on Systems, Man and Cybernetics (IEEE Cat. No.04CH37583).

[50]P. Cash and A. Maier, "Prototyping with your hands: the many roles of gesture in the communication of design concepts", Journal of Engineering Design, vol. 27, no. 1-3, pp. 118145, 2016.

[51]L. Richland, "Linking Gestures: Cross-Cultural Variation During Instructional Analogies", Cognition and Instruction, vol. 33, no. 4, pp. 295-321, 2015.

[52]L. Lanyon and M. Rose, "Do the hands have it? The facilitation effects of arm and hand gesture on word retrieval in aphasia", Aphasiology, vol. 23, no. 7-8, pp. 809-822, 2009.

[53]K. Zhong, J. Kang, S. Qin and H. Wang, "Rapid 3D conceptual design based on hand gesture", 2011 3rd International Conference on Advanced Computer Control, 2011.

[54]S. Bodiroža, H. Stern and Y. Edan, "Dynamic gesture vocabulary design for intuitive humanrobot dialog", Proceedings of the seventh annual ACM/IEEE international conference on Human-Robot Interaction - HRI '12, 2012.

[55]M. van Beurdena, W. IJsselsteijna and K. Hopfb, "User centered design of gesture-based interaction technology", 2011 3DTV Conference: The True Vision - Capture, Transmission and Display of 3D Video (3DTV-CON), 2011.

[56]I. Hwang, H. Kim, J. Cha, C. Ahn, K. Kim and J. Park, "A gesture based TV control interface for visually impaired: Initial design and user study", 2015 21st Korea-Japan Joint Workshop on Frontiers of Computer Vision (FCV), 2015. 
[57]O. Luzanin and M. Plancak, "Hand gesture recognition using low-budget data glove and cluster-trained probabilistic neural network", Assembly Automation, vol. 34, no. 1, pp. 94-105, 2014.

[58]W. Zhu, L. Liu, S. Yin, S. Hu, E. Tang and S. Wei, "Motion-sensor fusion-based gesture recognition and its VLSI architecture design for mobile devices", International Journal of Electronics, vol. 101, no. 5, pp. 621-635, 2013.

[59]Y. Zhiqi, "Gesture learning and recognition based on the Chebyshev polynomial neural network", 2016 IEEE Information Technology, Networking, Electronic and Automation Control Conference, 2016.

[60]R. Agrawal and N. Gupta, "Real Time Hand Gesture Recognition for Human Computer Interaction", 2016 IEEE 6th International Conference on Advanced Computing (IACC), 2016.

[61]"Imperial Computer Vision \& Learning Lab (ICVL)", Iis.ee.ic.ac.uk, 2017. [Online]. Available: http://www.iis.ee.ic.ac.uk/ComputerVision/. [Accessed: 20 - Oct- 2016].

[62]Y. Wei, "Yichen Wei at Microsoft Research", Microsoft Research, 2017. [Online]. Available: http://research.microsoft.com/en-us/um/people/yichenw/handtracking/. [Accessed: 20 - Oct2016].

[63]L. Liu, "SKIG", Lshao.staff.shef.ac.uk, 2017. [Online]. Available: http://shao.staff.shef.ac.uk/data/SheffieldKinectGesture.htm. [Accessed: 20 - Oct- 2016].

[64]I. Bullock, T. Feix and A. Dollar, "The Yale Human Grasping Data Set", Eng.yale.edu, 2017. [Online]. Available: http://www.eng.yale.edu/grablab/humangrasping/. [Accessed: 20 - Oct2016].

[65]P. Rogers, J. Rogers, and Y. Rogers. Interaction Design: Beyond Human-computer Interaction. New York, NY: J. Wiley \& Sons, 2002. Print.

[66]"Hand gesture database", Gti.ssr.upm.es, 2017. [Online]. Available: https://www.gti.ssr.upm.es/data/HandGesture_database.html. [Accessed: 07-Apr- 2017].

[67]M. Kawulok, "Hand pose estimation", Database for hand gesture recognition, 2017. [Online]. Available: http://sun.aei.polsl.pl/ mkawulok/gestures/. [Accessed: 08- Apr- 2017].

[68"Welcome to Myo Support", Myo Support, 2017. [Online]. Available: https://support.getmyo.com/hc/en-us. [Accessed: 01- Mar- 2017].

[69]D. Cunningham and G. Romanes, Cunningham's textbook of anatomy, 1st ed. London: Oxford University Press, 1966. 